\title{
Paleoceanographic history of the Japan Sea over the last 9.5 million years inferred from radiolarian assemblages (IODP Expedition 346 Sites U1425 and U1430)
}

\author{
Kenji M. Matsuzaki ${ }^{1,2^{*}}$, Takuya Itaki ${ }^{3}$, Ryuji Tada ${ }^{1}$ and Shin-ichi Kamikuri ${ }^{4}$
}

\begin{abstract}
Previous studies showed that the evolution of the Japan Sea paleoceanography since the Miocene has been influenced by the regional tectonism (e.g., opening/closing of the connecting seaways) and regional/global climate. In the Japan Sea, Expedition 346 of the Integrated Ocean Drilling Program (IODP) retrieved core sediments dating back to the Miocene at two sites (U1425 and U1430). In this study, we reconstruct shallow-to-deep-water hydrography of the Japan Sea during the Mio-Pliocene based on radiolarian assemblages at Sites U1425 and U1430 considering the local tectonism and changes in global/regional climate. Our data suggest that glacioeustatic sea-level changes have probably had an influence on the local paleoceanography between 9.5 and $7.0 \mathrm{Ma}$. Indeed, warm water probably flowed from the North Pacific into the Japan Sea when sea level was high via shallow central and eastern seaways. In addition, the sill depth of the northern seaway was probably close to $1000 \mathrm{~m}$ between 9.5 and $7.8 \mathrm{Ma}$ and had probably allowed inflow of oxygen minimum zone water from the North Pacific to the Japan Sea when sea level was high. In contrast, our data imply that Cycladophora nakasekoi, an endemic species to the Japan Sea, dominated between 9.5 and $7.3 \mathrm{Ma}$ when sea level was low. Our data also suggest a progressive shoaling of the sill for the period since $7.8 \mathrm{Ma}$ and that global climatic events such as such the late Miocene cooling (7.5-5.5 Ma) and the early Pliocene warmth have had a sustained influence on the Japan Sea. During the mid-Pliocene, a deep cooling of the subsurface to intermediate water of the Japan Sea likely occurred because species related to subarctic subsurface to intermediate waters were dominant between 5 and $3.8 \mathrm{Ma}$. The Northern Hemisphere Glaciation (ca. 3.0-2.7 Ma) and Mid-Pleistocene Transition (1.2-0.8 Ma) have both likely intensified the cooling of the Japan Sea.
\end{abstract}

Keywords: Japan Sea, Integrated Ocean drilling program, Paleoceanography, Sea-level changes, Sill depths, Oxygen minimum zone, Radiolarians

\section{Introduction}

The Japan Sea is a back-arc basin opened by continental rifting during the late Oligocene to middle Miocene Epoch, ca. 28-13 million years ago (Ma) (e.g., Tamaki et al. 1992; Jolivet et al. 1994). Currently, the Japan Sea is connected to neighboring seas by four shallow straits: the Tsushima Strait (sill depth of $130 \mathrm{~m}$ ) connecting to the

\footnotetext{
* Correspondence: km.matsuzaki@aori.u-tokyo.ac.jp

${ }^{1}$ Department of Earth and Planetary Science, Graduate School of Science, The University of Tokyo, 7-3-1 Hongo, Bunkyo-ku, Tokyo 113-0033, Japan ${ }^{2}$ Present address: Atmosphere and Ocean Research Institute, The University of Tokyo, 5-1-5 Kashiwanoha, Kashiwa, Chiba 277-8564, Japan Full list of author information is available at the end of the article
}

East China Sea, the Tsugaru Strait (sill depth of $130 \mathrm{~m}$ ) connecting to the Pacific, and the Soya and Mamiya Straits (sill depths of 55 and $12 \mathrm{~m}$, respectively) connecting to the Sea of Okhotsk (Fig. 1). Therefore, water exchanges are restricted only to the surface waters of the East China Sea, Northwest Pacific, and Sea of Okhotsk, and intrusion of intermediate and deep waters is prevented (e.g., Gamo et al. 2014). This semi-isolation of the Japan Sea leads to formation of its own deep water, the so-called Japan Sea Proper Water, by cooling of the Tsushima Warm Current (TWC) (e.g., Gamo et al. 2014). The Japanese islands and Sakhalin Island, which bound the eastern margin of the Japan Sea, are characterized by 


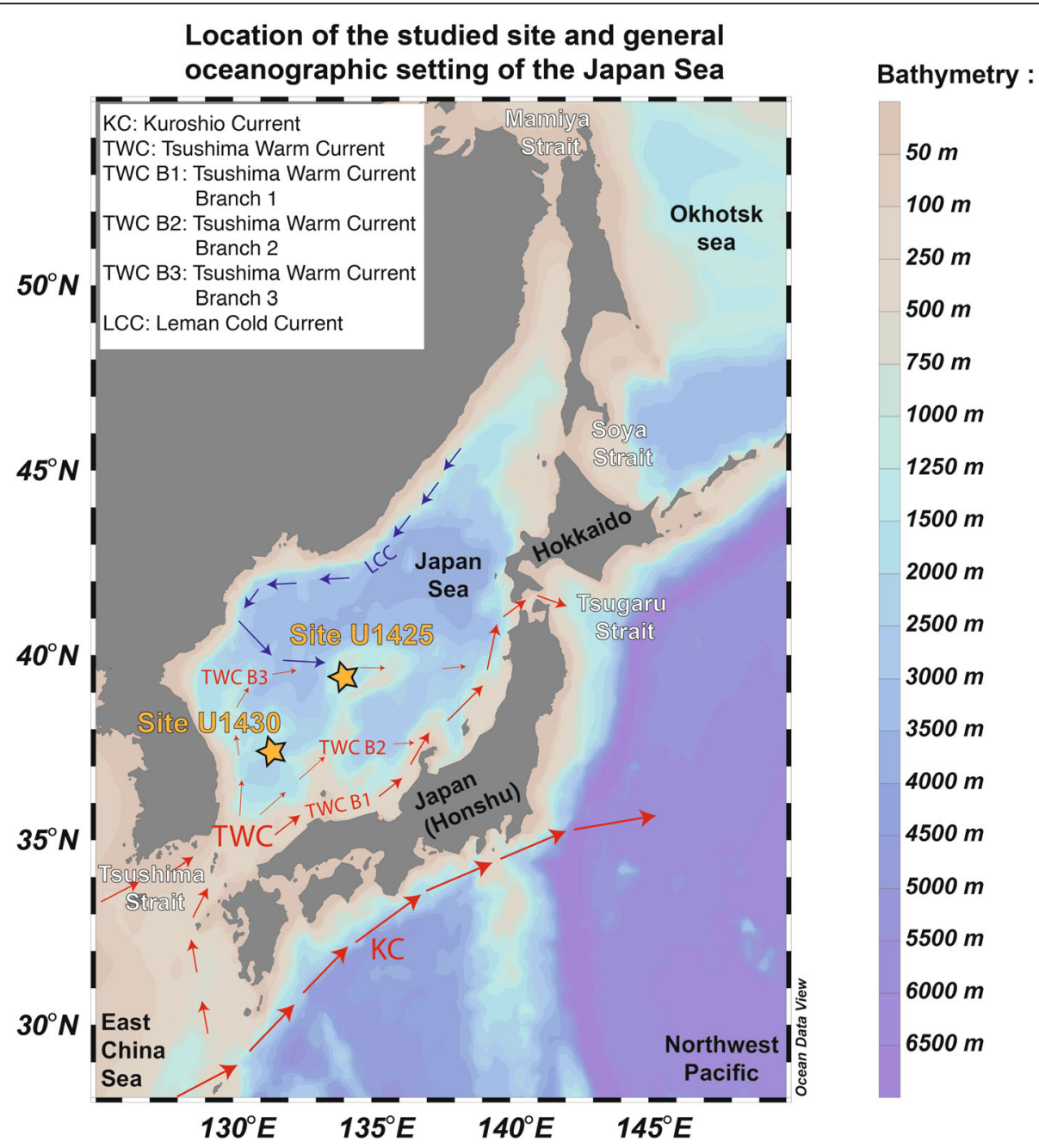

Fig. 1 Location of the study sites and the local oceanographic setting of the Japan Sea. The map was generated by the software Ocean Data View 4 of Schlitzer (2016)

active tectonism, which drastically modified the Japan Sea paleogeography, such as the sill depths of the key straits during the Neogene (e.g., Iijima and Tada 1990; Jolivet et al. 1994; Kosaka et al. 1992; Kano et al. 1991; Suzuki 1989; Taira 2001).

During the middle Miocene, a sedimentary basin called the Fossa Magna was formed in central Japan because of back-arc rifting associated with the opening of the Japan Sea (e.g., Iijima and Tada 1990). The paleobathymetry of this basin evolved from deep water depths in the middle Miocene (middle bathyal sedimentary facies) to shallow water depths in the late Miocene (shallow-water sedimentary facies) (e.g., Fujii et al. 1992; Iijima and Tada 1990; Kobayashi and Tateishi 1992; Kosaka et al. 1992). The beginning of a compressional stress regime in Northeast Japan due to the collision of the Izu-Bonin Arc since the middle Miocene may have caused the shoaling and closure of this seaway ca. 8-7 Ma (e.g., Sato 1994; Sato et al. 2004;
Iijima and Tada 1990; Yoshida et al. 2013). In addition, during the late Miocene, a few shallow seaways (about $200 \mathrm{~m}$ deep) probably existed in Northeast Japan because of subsidence of the Tohoku-Hokkaido area during the late Miocene (Sato 1994; Yoshida et al. 2013). However, these subsided areas were also likely uplifted because of the compressional stress regime, which started ca. 8-7 Ma (Sato 1994; Yoshida et al. 2013) (Fig. 2). At the same time, the south of the Japan Sea was likely isolated by land from the North Pacific because no marine fossils and microfossils were recorded from the late Miocene (ca. $10 \mathrm{Ma}$ ) to the early Pliocene (ca. $4 \mathrm{Ma}$ ) along the eastern edges of the Yellow and South China seas (e.g., Iijima and Tada 1990; Kano et al. 1991; Wang et al. 2014) (Fig. 2). In the northern Japan Sea (corresponding to the southern to central part of Hokkaido Island), several geological studies suggested that a bathyal environment prevailed since the beginning of the late Miocene until the late Pliocene (e.g., Fukusawa 1988; 


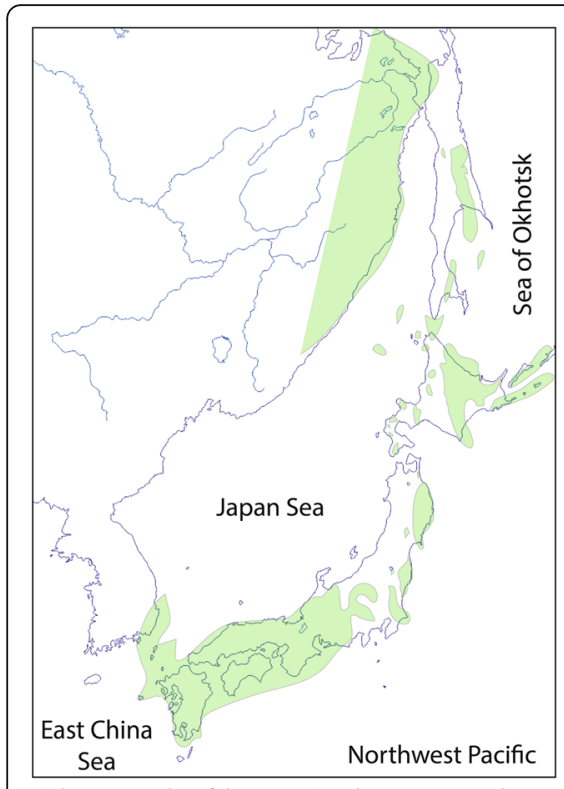

Paleogeography of the Japan Sea between 10 and $7 \mathrm{Ma}$

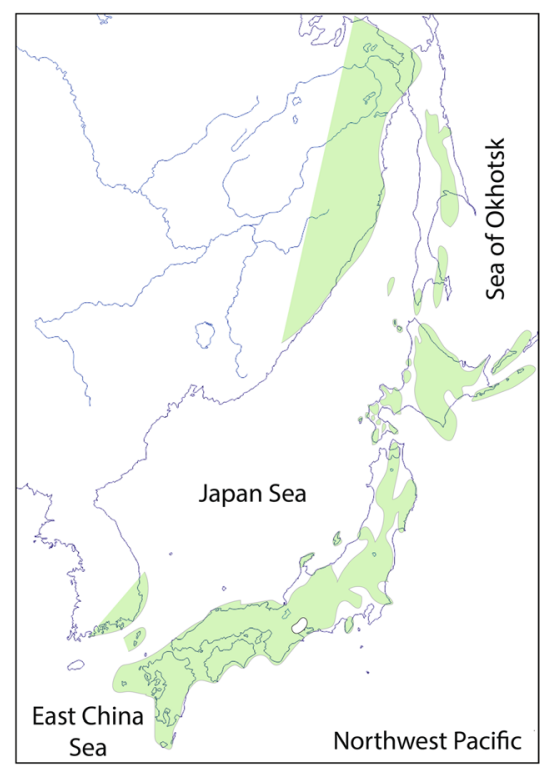

Paleogeography of the Japan Sea between 7 and $3 \mathrm{Ma}$

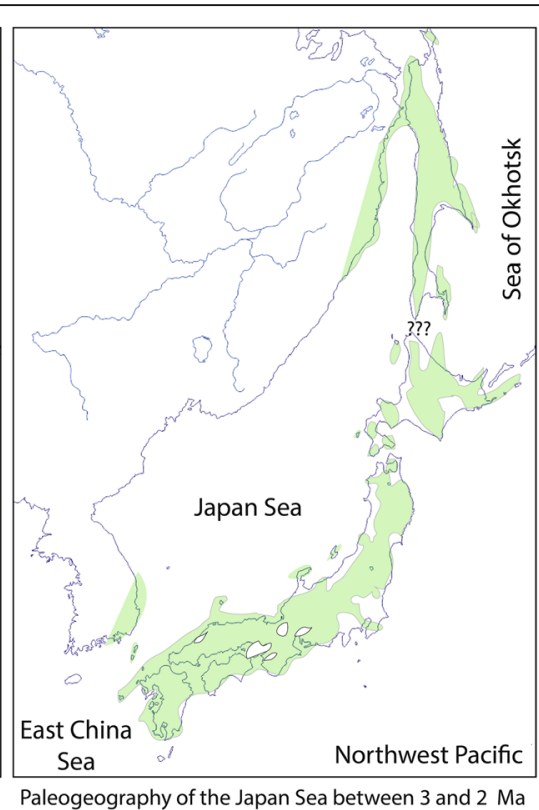

Fig. 2 Evolution of the Japan Sea since 10.5 Ma and its paleomaps (based on Fukusawa 1988; Fujii et al. 1992; lijima and Tada 1990; Sato 1994; Kano et al. 1991; Kharakhinov 2010; Kobayashi and Tateishi 1992; Kosaka et al. 1992; Sagayama, 2002; Yahata 2002; Yoshida et al. 2013). The base lines of the coasts and hydrography came from http://d-maps.com

Iijima and Tada 1990; Itaki 2016; Kosaka et al. 1992; Kano et al. 1991; Sagayama 2002; Suzuki 1989; Ogasawara 1994; Yahata 2002) (Fig. 2). At the northernmost Japan Sea, in the area presently around the Sakhalin and Tatar straits, tectonic studies suggested that a neritic environment prevailed from the middle Miocene to the end of the late Miocene (Kharakhinov 2010) (Fig. 2). A southward shift of the Okhotsk plate likely caused a southward migration of the Kuril basin and a progressive shoaling and closure of the Sakhalin seaways during the early to late Pliocene (Takeuchi et al. 1999; Kharakhinov 2010).

Paleoceanographic conditions in the Japan Sea are known to have been sensitive to orbital and suborbital forcings and glacioeustatic sea-level changes during the middle to late Pleistocene (e.g., Tada et al. 1999; Itaki et al. 2007; Kido et al. 2007). Currently, the TWC is the unique current flowing into the Japan Sea and controlling its oceanographic condition (Gamo et al. 2014) (Fig. 1). After entering, the TWC bifurcates into three branches at the southwestern part of the Japan Sea near the Tsushima Strait (Hase et al., 1999) (Fig. 1). The first branch of the TWC (TWC B1) flows along the west coast of Honshu Island (Fig. 1). The second branch of the TWC (TWC B2) flows along the Korean peninsula before rejoining TWC $\mathrm{B} 1$, and the third branch of the TWC (TWC B3) flows along the Korean Peninsula northward to form the Leman Cold Current (LCC in Fig. 1). During the Pleistocene, the amplitude of glacioeustatic sea-level variations caused by orbital cycles was about $100 \mathrm{~m}$ (e.g., Miller et al. 2005; Siddall et al.
2003; Yokoyama et al. 2001; Itaki 2007; Itaki et al. 2004). Because the Tsushima Strait has a shallow sill depth (about $130 \mathrm{~m}$ ), a sea-level variation of about $100 \mathrm{~m}$ restricted flow of the TWC into the Japan Sea, leading to a decrease in surface water salinity and drastically reducing the local deep-water ventilation, which resulted in euxinic bottom-water conditions (e.g., Kido et al. 2007; Oba et al. 1991; Tada et al. 1999).

For the Mio-Pliocene, Tada (1994) compiled the published sedimentological and diatom data of Koizumi (1992a, 1992b) and Tada and Iijima (1992) related to the paleoceanographic history of the Japan Sea and discussed the sill depth of the connecting straits that could have influenced exchanges between the Japan Sea and the North Pacific. Tada (1994) suggested that sediment deposition in the Japan Sea began at ca. $20 \mathrm{Ma}$, and the sea was open to the south, east, and north until ca. 10.5 Ma. Warm surface water and relatively oxic bottom water conditions prevailed until ca. 15-14 Ma, when a drastic cooling was recorded. Mollusca fossil records also suggest this kind of cooling and indicate that temperate water prevailed since ca. 13 Ma (Chinzei 1978; Ogasawara 1994). According to recent studies, this cooling is closely related to the mid-Miocene global cooling events (15-14 Ma), including the East Antarctic Ice Sheet expansion and stabilization (Holbourn et al. 2013, 2014; Zachos et al. 2001, 2008). The southern strait closed ca. 10.5 Ma, which enhanced the cooling of the Japan Sea (Tada 1994; Ogasawara 1994). In contrast, sedimentary, microfossil facies, and chemical data suggest that the Japan Sea bottom water was oxygen-deficient from 
18 to ca. $6.5 \mathrm{Ma}$ (Hanagata 2003, 2006; Tada 1994; Yamamoto et al. 2005). Tada (1994) suggested that the origin of the oxygen-poor water was related to inflow of oxygen minimum zone (OMZ) water of the adjacent North Pacific. The presence of this OMZ in the North Pacific during the Miocene may be controvertible. However, Woodruff and Savin (1989) used measurement of $\delta^{13} \mathrm{C}$ in the benthic foraminifer Cibicidoides to report a very low $\delta^{13} \mathrm{C}$ value for the North Pacific between latitudes $20^{\circ}$ and $40^{\circ}$ at water depths between ca. 1000 and $3000 \mathrm{~m}$ during the middle and late Miocene. This implied the presence of a layer with low dissolved oxygen content in the North Pacific between 1000 and $3000 \mathrm{~m}$ at that time (e.g., Woodruff and Savin 1989). Additionally, radiolarian studies were conducted in the context of the Deep-Sea Drilling Program (DSDP) and Ocean Drilling Program (ODP) (namely, legs 31, and 127 and 128). These studies mainly produced biostratigraphic reports (Alexandrovich 1992; White and Alexandrovich 1992; Ling 1992). Kamikuri and Motoyama (2007) showed that, at DSDP Site 302, radiolarian species related to the North Pacific deep water occurred in the Japan Sea between 8 and ca. $3 \mathrm{Ma}$, which suggests inflow of deep water of the North Pacific into the Japan Sea. These findings showed that the hypothesis proposed by Tada (1994) for that time is plausible, although some concern remains about the ecology of the radiolarian species associated with the North Pacific deep water. For the late Pliocene, few studies have reconstructed paleoceanographic conditions of the Japan Sea. Most of them agree that, during the late Pliocene, cold surface water prevailed in the northern part of the Japan Sea, while warm water from the southern strait (Tsushima Strait) influenced the sea only during interglacial periods (e.g., Kitamura and Kimoto 2006; Kitamura et al. 2001).

\section{Problem addressed here}

Our knowledge about global and regional climatic events has improved greatly. Recent studies stressed the importance of the monsoon on the global climate and ocean and also stressed the importance of the uplift of the Tibetan-Himalayan Plateau at the Oligocene/Miocene boundary in establishing the monsoon (e.g., Betzler et al. 2017; Clift 2017; Holbourn et al. 2018; Tada et al. 2016). The monsoon has an influence on the carbon cycle by controlling chemical weathering and thus strongly affecting the global climate (Raymo and Ruddiman 1992; Tada et al. 2016). During the late Miocene between 7 and $5.5 \mathrm{Ma}$, a period of global cooling was reported for the middle to high latitudes of both hemispheres, the so-called late Miocene cooling event (Herbert et al. 2016). Recent studies suggested that the East Asian winter monsoons (EAWM) were probably stronger at that time, and thus the EAWM probably played an important role in the late Miocene cooling event (Holbourn et al. 2018). Recent studies suggested that the beginning of the shoaling of the Panama Isthmus to a few hundred meters of water depth probably caused changes in the meridional deep ocean circulation (Butzin et al. 2011). Then, during the Pliocene, the final closure of the Panama Isthmus caused drastic changes in ocean salinity/heat distributions that may have triggered the Northern Hemisphere Glaciation (NHG) (e.g., Cortese et al. 2004; Haug et al. 2001; Karas et al. 2017; Motoi et al. 2005; Sandoval et al. 2017; Steph et al. 2006; Raymo 1994; Stroynowski et al. 2015). These changes may have caused an episodic strong Atlantic meridional overturning circulation between 4.5 and 3.8 Ma (Karas et al. 2017). In addition, although it remains under debate, between 16.4 and $8 \mathrm{Ma}$, the Indonesian seaway, which allowed water mass exchanges between the Northern Pacific and the Indian Ocean, closed progressively and probably caused the formation of a proto-Kuroshio Current (e.g., Kennett et al. 1985; Ogasawara et al. 2008).

Consequently, the reconstruction of changes in the Japan Sea paleoceanography during the Mio-Pliocene is crucial for a better understanding of how the Japan Sea was affected by these global tectonic and climatic events.

\section{Objective and strategy}

In this study, we analyzed deep-sea sediments of the Japan Sea collected during Expedition 346 of the Integrated Ocean Drilling Program (IODP) to reconstruct past hydrographic conditions of the Japan Sea during the Mio-Pliocene. Sediment cores were collected from seven sites covering wide latitudinal and depth ranges in the Japan Sea. We selected samples collected at Sites U1425 and U1430 in the central and southcentral parts of the Japan Sea during IODP Expedition 346 because hemipelagic sediments covering the middle Miocene to Pliocene were retrieved (Tada et al. 2015a, 2015b). We analyzed 154 sediment core samples collected at Site U1425 (about $2000 \mathrm{~m}$ deep) and 51 samples collected at Site U1430 (about $1000 \mathrm{~m}$ deep) to reconstruct past paleoceanography of the Japan Sea since the late Miocene. In the Japan Sea, the present calcite compensation depth (CCD) is about $2000 \mathrm{~m}$, and dissolution of planktic foraminifera begins at a depth of about $1400 \mathrm{~m}$ (Ujiie and Ichikura 1973). Lee et al. (2000) reported that the CCD was likely shallower during the Mio-Pliocene. These data show that the use of paleoceanographic proxies based on calcareous microfossils is not necessarily suitable for the Japan Sea, particularly for sites with water depths deeper than $1000 \mathrm{~m}$ (Sites U1425 and U1430). Consequently, the use of assemblages of siliceous microfossils appears to be a unique alternative to reconstructing oceanographic conditions of the shallow to deep waters. Because radiolarians are a unique siliceous microfossil group inhabiting shallow to 
deep waters, we analyzed their assemblages and changes over time to reconstruct hydrographic changes of the shallow to deep waters since the Miocene.

\section{Methods/experimental}

\section{Sites studied}

In this study, we analyzed sediment core samples collected from Sites U1425 and U1430 drilled during IODP Expedition 346 (2013). However, because a hiatus is present between 7.2 and ca. $4.5 \mathrm{Ma}$ at Site U1430 (Kamikuri et al. 2017; Tada et al. 2015b), most of the analysis was conducted on samples from Site U1425. In this study, we only used data from Site U1430 to provide supporting information.

Site U1425 is located in the central part of the Japan Sea, in the middle of the Yamato Bank (1909 m water depth) at $39^{\circ} 29.44^{\prime} \mathrm{N}$ and $134^{\circ} 26.55^{\prime} \mathrm{E}$ (Tada et al. 2015a). The drilling at Site U1425 recovered a total of $417.5 \mathrm{~m}$ of sediment (recovery rate of $98 \%$ ). The description of the sediment core lithology at Site U1425 given below follows that of Tada et al. (2015a).

The lithology of Mio-Pliocene sediments at this site can be divided into three major lithological units, and each is subdivided into two subunits (e.g., IA and IB)
(Figs. 3 and 4). Unit III is the older and deeper lithological unit (> $262 \mathrm{~m}$ CCSF-D; > 7.419 Ma) (CCSF-D is an abbreviation for core composite depth below seafloor). This unit consists of clay, diatom-rich clay, and diatom ooze. This unit can be divided into two subunits based on the degree of lithification (IIIA and IIIB). Subunit IIIB corresponds to the depth interval deeper than $356 \mathrm{~m}$ CCSF-D (> 9.528 Ma), and it is characterized by gray siliceous claystone with occasional parallel laminations and burrows. Lithological subunit IIIB is not discussed in this study, because it contained no biogenic silica. Subunit IIIA corresponds to the depth interval between ca. 356 and $262 \mathrm{~m}$ CCSF-D (9.528-7.419 Ma) (Figs. 3 and 4). This subunit is characterized by decimeter- to meter-scale cyclic alternation of dark gray diatom ooze and moderate to heavily bioturbated clayey diatomaceous ooze. A fine lamination of diatom ooze occurring within a dark organic-rich layer extending to a thickness of about $1.5 \mathrm{~m}$ has been recognized at $275 \mathrm{~m}$ core depth below seafloor (CSF-A) (Tada et al. 2015a), which corresponds to about $287 \mathrm{~m}$ CCSF-D (Irino et al. 2018). Based on the age model of Kamikuri et al. (2017), these laminations occurred about $8 \mathrm{Ma}$. Lithological unit II corresponds to a depth interval between ca. 262 and

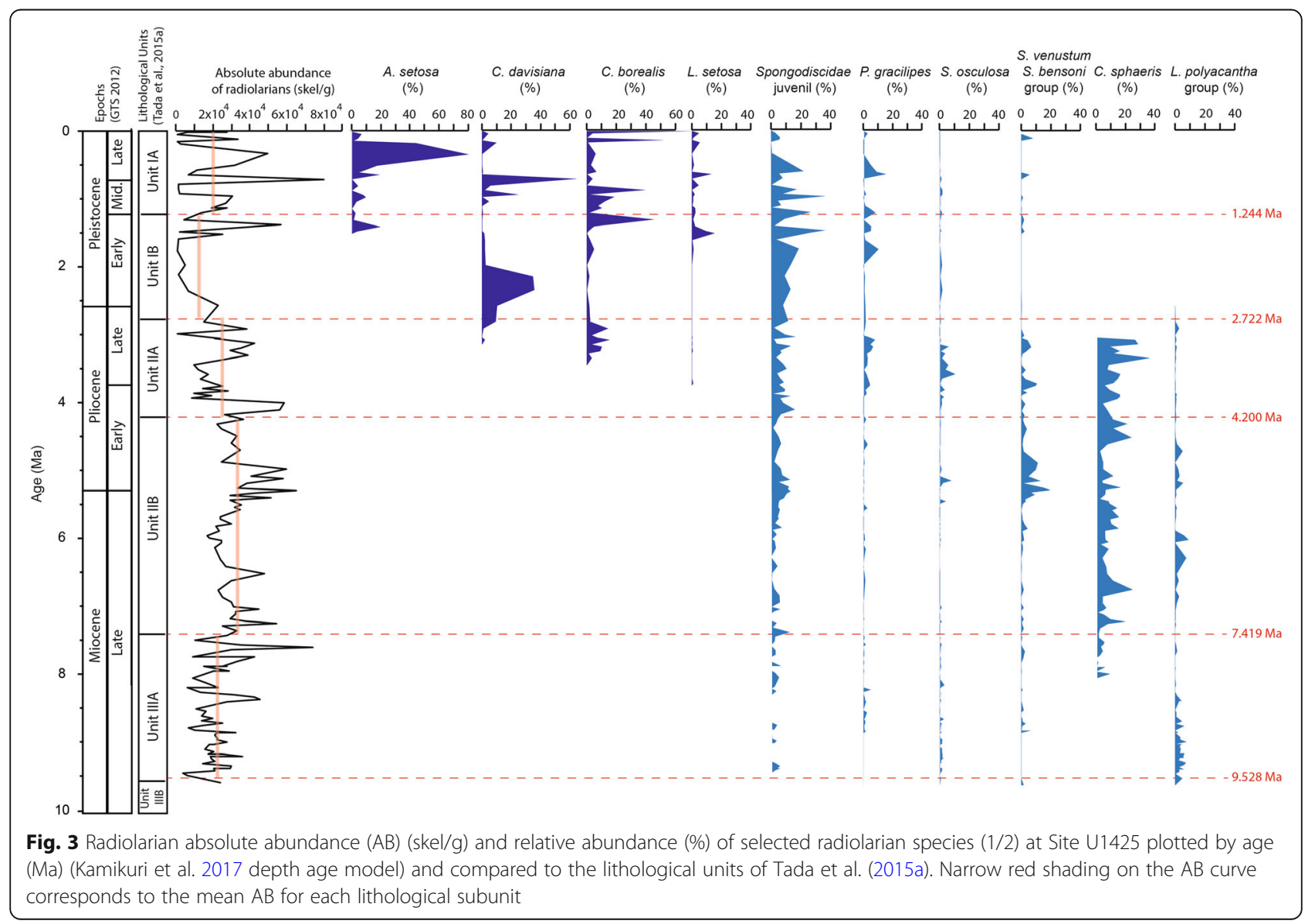




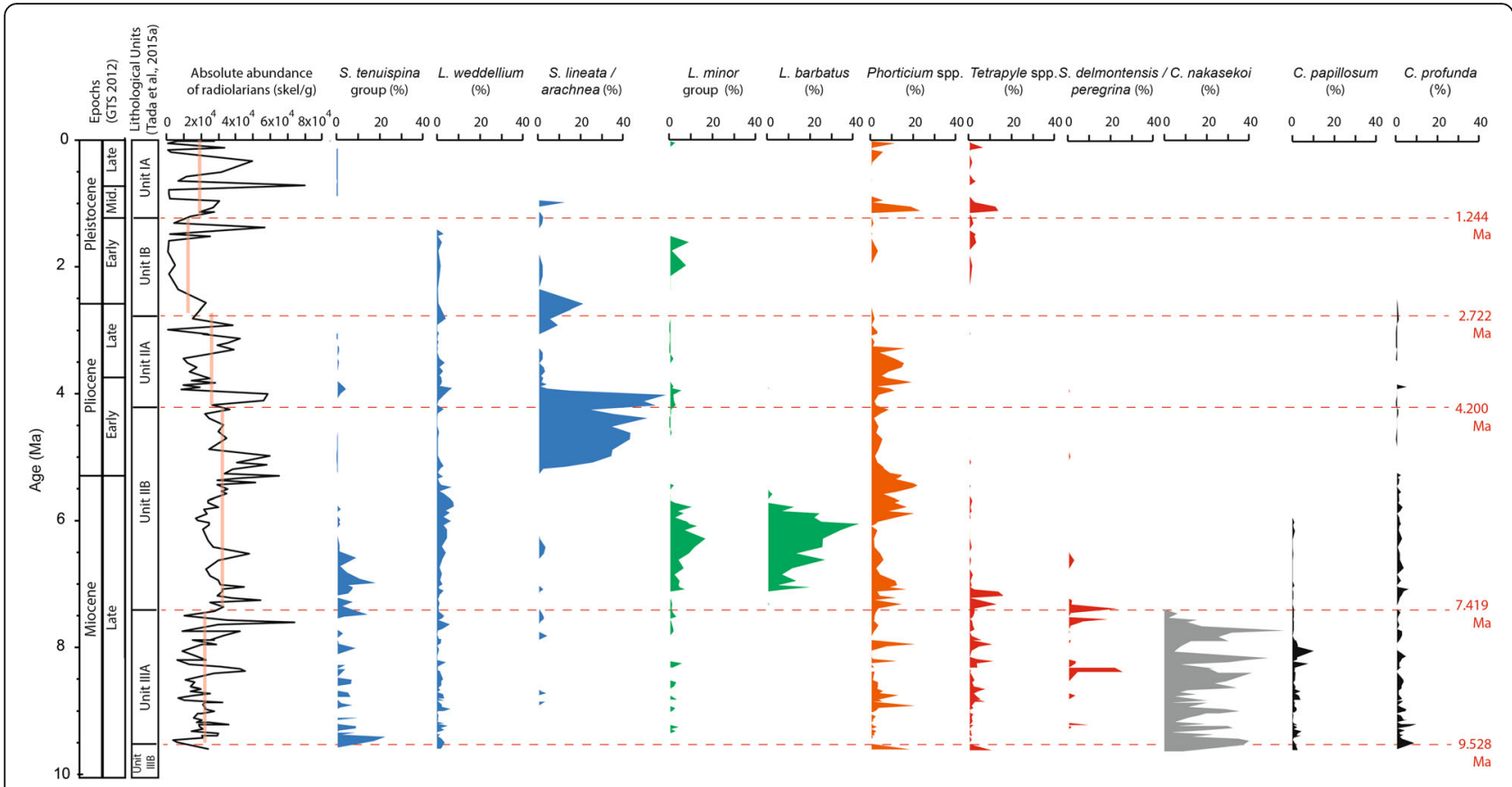

Fig. 4 Radiolarian absolute abundance (AB) (skel/g) and relative abundance (\%) of selected radiolarian species (2/2) at Site U1425 plotted by age (Ma) (Kamikuri et al. 2017 depth age model) and compared to the lithological units of Tada et al. (2015a). Narrow red shading on the AB curve corresponds to the mean $A B$ for each lithological subunit

99 m CCSF-D (7.419-2.722 Ma) (Figs. 3 and 4). This unit consists of diatomaceous clay and diatomaceous ooze that is moderately to heavily bioturbated. Unit II can be distinguished from unit III by a decrease in terrigenous matter. This unit is divided into two subunits (IIA and IIB) based on the diatom content and intensity of bioturbation. Subunit IIB (ca. 262-137 m CCSF-D; 7.419-4.200 Ma) is dominated by brownish diatom ooze. Diatom content (70-90\% of the smear slide) is a key component for the recognition of this subunit. Subunit IIA (ca. 137-99 m CCSF-D; 4.200-2.722 Ma) mainly consists of brownish-greenish diatom-rich clay. In general, the sediments of subunit IIA are heavily bioturbated. Unit I corresponds to a depth interval between ca. 99 and $0 \mathrm{~m}$ CCSF-D (since $2.722 \mathrm{Ma}$ ) (Figs. 3 and 4). Unit I consists of clay and silty clay with small amounts of diatom-rich clay, while foraminifer-bearing clay is rare. This unit is divided into two subunits (IA and IB) based on the frequency of the color alternations (dark and light layer) and the intensity of bioturbation. Subunit IB (ca. 99-51 CCSF-D; 2.722-1.244 Ma) consists of light greenish gray clay with a low frequency of dark and light color alternation. Subunit IA (ca. 51-0 m CCSF-D; since $1.244 \mathrm{Ma}$ ) is dominated by clay, small amounts of diatomaceous-bearing clay, and tephras. This subunit is also characterized by a pronounced decimeter-scale alternation of light and dark layers.

Site U1430 is located on the southern slope of the eastern South Korean Plateau (1083 m below sea level) at $37^{\circ} 54.16^{\prime} \mathrm{N}$ and $131^{\circ} 32.25^{\prime} \mathrm{E}$. As above, the lithology of the sediment cores at Site U1430 is described following Tada et al. (2015b) (Fig. 5). The lithology of this site consists of clayey silt, silty clay, nannofossil ooze, diatom ooze, claystone, and sandstone. This site can be divided into four units (I to IV), and each of units I to III is further divided into two subunits based on sediment composition and relative abundances of biosiliceous and siliciclastic fractions. However, subunit IIB and unit IV correspond to glauconite sandstone intervals with no biogenic silica, so these units are not discussed further. Subunit IIIA corresponds to the depth interval between ca. 256 and 103 m CCSF-D (11.714-7.891 Ma) (Fig. 5). This subunit is characterized by decimeter- to meter-scale alternating layers of heavily bioturbated, relatively dark gray diatomaceous ooze and very dark gray diatom-rich clay. Lithological unit II corresponds to a depth interval between ca. 103 and $60 \mathrm{~m}$ CCSF-D (7.891-3.003 Ma) (Fig. 5). This unit consists of green silty clay, olive gray diatom-rich silty clay, and dark olive-gray diatom ooze. This unit is divided into two subunits (IIA and IIB) based on the diatom content. Preliminary studies using biostratigraphic data suggest that subunit IIB (103-76 m CCSF-D; 7.891-4.100 Ma) corresponds to a hiatus (Tada et al. 2015b; Kamikuri et al. 2017). Subunit IIA (ca. 76-60 m CCSF-D; 4.100$3.003 \mathrm{Ma})$ mainly consists of meter-scale alternating heavily bioturbated dark olive-gray diatom-bearing clay and very dark gray diatom ooze. Unit I differs from unit 


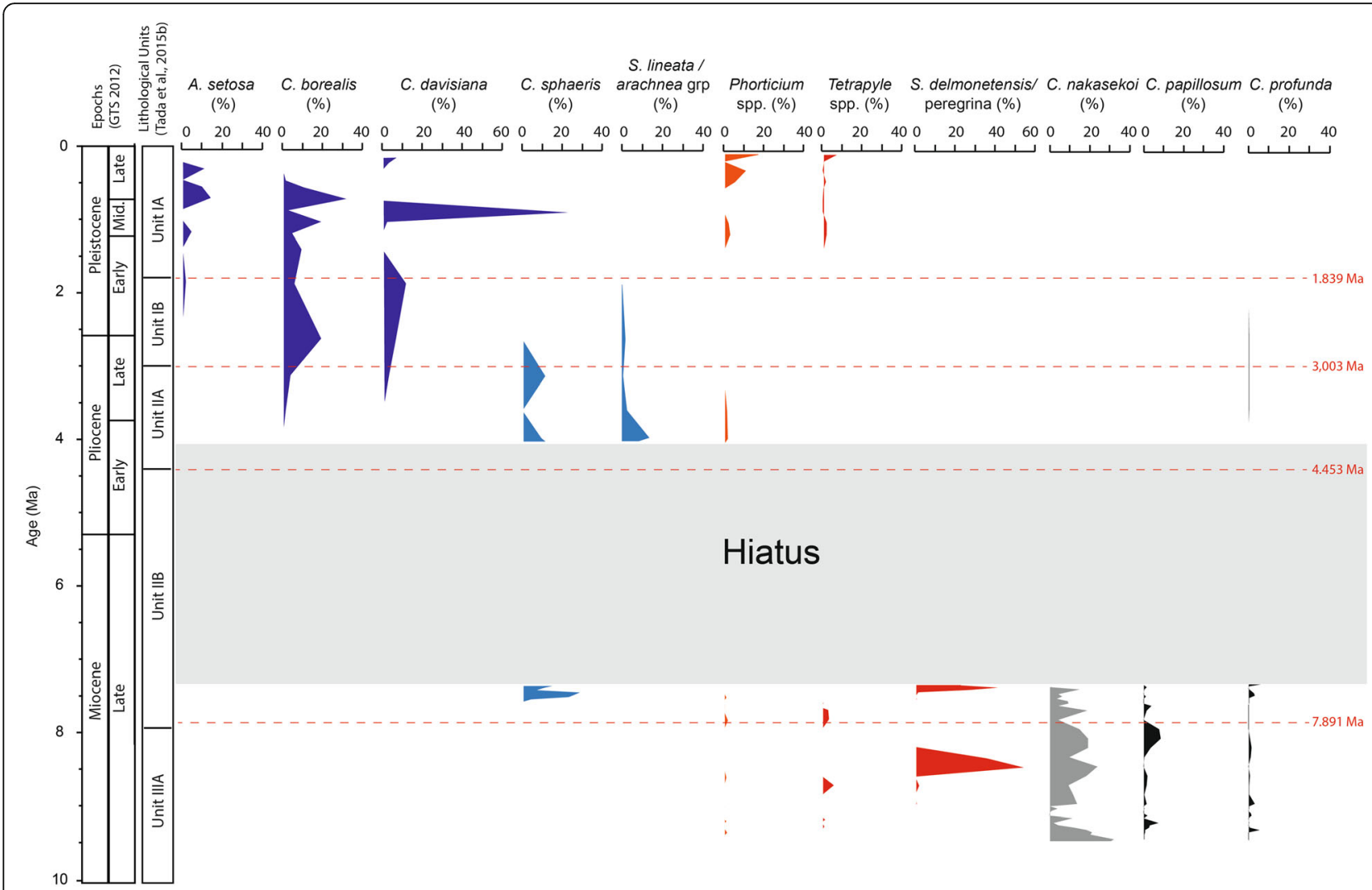

Fig. 5 Relative abundance (\%) of selected radiolarian species at Site U1430 plotted by age (Ma) (Kamikuri et al. 2017 depth age model) and compared to the lithological units of Tada et al. (2015b)

II by a decrease in biosiliceous content. This unit consists of alternating interbedded clayey silt, silt clay, foraminifer-rich diatom-bearing clayey silt, nannofossil ooze, and tephras. As for Site U1425, this unit is also divided into two subunits (IA and IB) based on the frequency of the color alternations and the intensity of bioturbation. Subunit IB (60-46 m CCSF-D; 3.003-1.839 Ma) consists of a low-frequency dark and light color alternation and increasing dominance of light gray clayey silt. Subunit IA (<46 m CCSF-D; since $1.839 \mathrm{Ma}$ ) consists of terrigenous grains, biogenic matter, pumice, and volcanic glass.

\section{Assemblage analysis}

In this study, we used the depth scale of Irino et al. (2018), who defined the splice and composite depths of all sediment cores retrieved during IODP Expedition 346 (CCSF-D patched-Ver. 2) (see details in Irino et al. 2018). Then, we analyzed changes in the radiolarian assemblage for 154 samples collected at about 3-m intervals from cores drilled at Site U1425 between 0 and $250 \mathrm{~m}$ CCSF-D and at about 2-m intervals between 250 and $360 \mathrm{~m}$ CCSF-D. To monitor changes in the Japan Sea hydrography, changes in the radiolarian assemblage and radiolarian absolute abundance $(\mathrm{AB}$, in skel/g) are useful tools for reconstructing hydrographic changes throughout the past $10 \mathrm{My}$. Two types of microscopic slides were prepared: a faunal slide (F-slide) for analyzing changes in the assemblage and a quantitative slide (Q-slide) for accurately estimating the $\mathrm{AB}$ of radiolarians in $1 \mathrm{~g}$ of dried sediment.

The 154 samples were freeze-dried and treated with diluted hydrogen peroxide (15\%) and hydrochloric acid (15\%) to remove organic and calcareous matter. The undissolved residue in each sample was washed over a sieve with $45 \mu \mathrm{m}$ mesh size. For mounting a Q-slide, we followed the procedure proposed by Itaki (2009) and Matsuzaki and Itaki (2016), which starts with transferring the washed undissolved residue to a beaker with $100 \mathrm{~mL}$ of water. The obtained solution is homogenized by mixing to settle the radiolarians randomly, after which a $0.5-\mathrm{mL}$ sample is withdrawn (Itaki 2009) and mounted onto an $18 \times 24-\mathrm{mm}$ cover slip to make a microscopic slide. All specimens visible on each Q-slide are counted. The $\mathrm{AB}$ of radiolarians is calculated as follows (Itaki 2009): $A=100 a / 0.5 g$, where $A$ is the estimated radiolarian $\mathrm{AB}$ (skel/g), $a$ is the number of radiolarian skeletons counted in the Q-slide, and $g$ is the weight of the freeze-dried sample. The remaining undissolved residue in the beaker was also homogenized by mixing to settle the radiolarians randomly and then 
mounted onto a $22 \times 40-\mathrm{mm}$ cover microscopic slide (F-slide) following the procedure of Itaki (2003). Examination of polycystine radiolarians was carried out under an optical microscope at total magnifications of 100$400 \times$. Sample examination continued until at least 400 radiolarians were identified in each sample, or until the sample material was exhausted. The identification followed the nomenclature of Itaki (2009), Matsuzaki et al. (2015a), and Zhang and Suzuki (2017) for extant species. For extinct species, we mainly followed the taxonomic nomenclature of Nigrini and Lombari (1984), Motoyama (1996), and Kamikuri (2010). Microphotographs of selected species are shown in Plate 1. When similar paleoceanographic responses were empirically known for similar species, these radiolarians were grouped for paleoceanographic analysis, as described below. We calculated the relative abundances of the encountered species as a percentage of all radiolarians in the sample. In this study, an average of 412 specimens was counted per sample. We also analyzed the radiolarian assemblage of 51 samples collected at 3.5-m intervals from Site U1430. However, because a hiatus of 2.7 Myr was encountered between 7.2 and ca. 4.5 Ma (Tada et al. 2015b; Kamikuri et al. 2017), only F-slides were mounted, and selected taxa were counted to strengthen the significance of the data obtained from Site U1425.

As mentioned above, a few aspects hamper the paleoceanographic reconstruction when it is based on changes in the microfossil assemblage for a time interval older than the Pleistocene, especially when including extinct species. Generally, knowledge of the vertical and geographical distributions of extinct species is limited. Fortunately, until the late Miocene, there are a few extant radiolarian species whose vertical and geographical distributions are known. Additionally, a few previous studies have clarified geographical distribution of Mio-Pliocene radiolarian species, which helped our reconstruction (Oseki and Suzuki 2009). In this context, we selected 21 extant/extinct species with known geographical distribution from the total assemblage in this study (Figs. 3, 4, and 5).

\section{Age model}

We followed the age-depth model of Kamikuri et al. (2017) for Sites U1425 and U1430 for the Mio-Pliocene and the age-depth model of Tada et al. (2018) for the Pleistocene, which relies on correlation of dark and light layers and estimates ages using tephras and magnetostratigraphic events. Details about the tie points and the sedimentation rates used in this study are shown in Tables 1 and 2.

\section{Results}

At both sites (U1425 and U1430), the preservation of radiolarian fossils fluctuated between good to moderate for the time interval between the late Miocene and late
Pliocene. During the Pleistocene, the preservation of radiolarians fluctuated between poor and good. A similar observation was also made by Kamikuri et al. (2017) and Tada et al. (2015a, 2015b).

\section{Site U1425 \\ Polycystine radiolarian absolute abundance (skel/g)}

Because the skeletons of polycystine radiolarians are composed of amorphous opaline silica, their $\mathrm{AB}$ can be used to constrain changes in opaline silica (e.g., De Wever et al. 2001) (Figs. 3 and 4).

In lithological subunit IIIA (9.52-7.41 Ma), the mean radiolarian $\mathrm{AB}$ is $2.24 \times 10^{4} \mathrm{skel} / \mathrm{g}$ and the standard deviation (SD) is $1.18 \times 10^{4} \mathrm{skel} / \mathrm{g}(N=54)$, while the means of radiolarian $\mathrm{AB}$ in lithological subunits $\mathrm{IIB}$ (7.41-4.20 Ma) and IIA (4.20-2.72 Ma) are $3.26 \times 10^{4} \mathrm{skel} / \mathrm{g}(\mathrm{SD}=1.10 \times$ $\left.10^{4} \mathrm{skel} / \mathrm{g} ; N=46\right)$ and $2.50 \times 10^{4} \mathrm{skel} / \mathrm{g}\left(\mathrm{SD}=1.41 \times 10^{4}\right.$ skel/g; $N=25$ ), respectively (Figs. 3 and 4 ). These data imply that although the mean radiolarian $\mathrm{AB}$ is lower in subunit IIIA than in subunits IIB and IIA, the radiolarian $\mathrm{AB}$ of subunit IIIA has a higher variability (subunit IIIA $_{\mathrm{SD}}>$ subunit IIA $_{\mathrm{SD}}>$ subunit IIB $_{\mathrm{SD}}$ ). These subunits are all characterized by a diatom ooze or diatom-rich clayey silt, suggesting high biogenic silica content. However, subunit IIIA, which has a lower mean value and higher SD, is characterized by a higher content of terrigenous matter (Tada et al. 2015a). Therefore, it is probable that the higher content of terrigenous matter reduced radiolarian productivity between 9.52 and 7.41 Ma (subunit IIIA). In addition, in subunit IIB (7.41-4.20 Ma), which is characterized by the highest radiolarian $\mathrm{AB}$ mean values and lowest $\mathrm{SD}$, the sediments are strongly bioturbated (Tada et al. 2015a). Previous studies demonstrated that highly bioturbated sediments imply oxic bottom water conditions in the Japan Sea during the Pleistocene (e.g., Watanabe et al. 2007). Therefore, it is probable that oxic bottom water conditions prevailed in the Japan Sea between 7.4 and 4.2 Ma, which probably allowed high productivity of radiolarians.

Lithological subunits IA and IB (1.2-0 Ma), which consist of clay and silty clay with small amounts of diatom-rich clay, are characterized by the lowest radiolarian $\mathrm{AB}$. Indeed, the means of radiolarian $\mathrm{AB}$ are $1.27 \times 10^{4} \mathrm{skel} / \mathrm{g}(\mathrm{SD}=$ $\left.1.62 \times 10^{4} \mathrm{skel} / \mathrm{g} ; N=11\right)$ and $2.01 \times 10^{4} \mathrm{skel} / \mathrm{g}(\mathrm{SD}=2.00 \times$ $10^{4} \mathrm{skel} / \mathrm{g} ; N=18$ ) for subunits $\mathrm{IB}$ and IA, respectively (Figs. 3 and 4). However, the SD estimated for subunit IA (since 1.2 Ma) is the highest since 9.5 Ma, which suggests high variability in radiolarian productivity since $1.2 \mathrm{Ma}$, which may be related to the glacioeustatic sea-level variation caused by glacial/interglacial cyclic climate changes (e.g., Elderfield et al. 2012; Lisiecki and Raymo 2005).

\section{Relative abundances of dominant species}

We have defined a species or species group as dominant if their relative abundance exceeded $30 \%$ of the total 

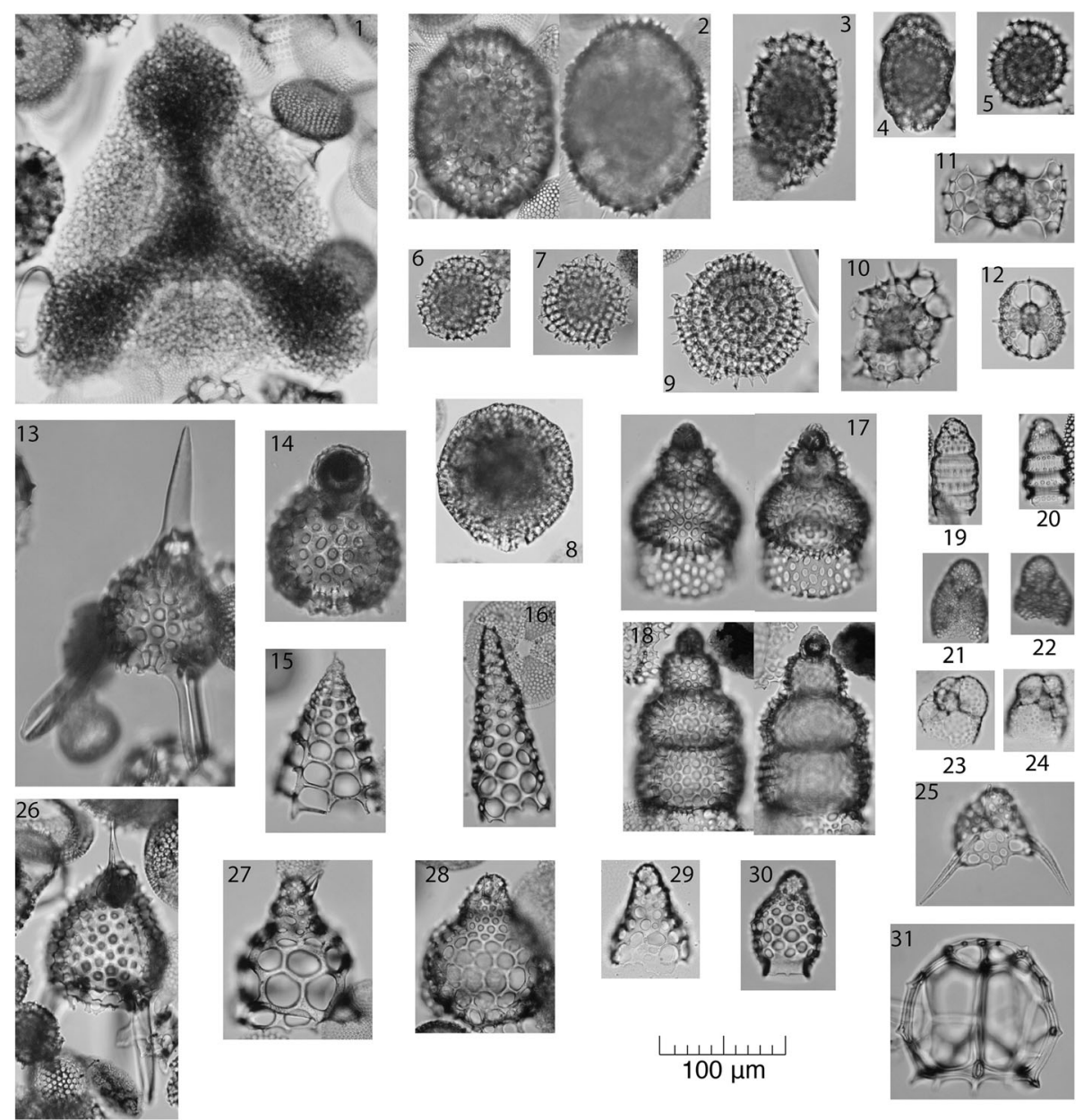

Plate 1 Photomicrographs of select radiolarian species used in this study: 1. Dictyocoryne profunda Ehrenberg (sample 346-U1425D-1H1-12$14 \mathrm{~cm}$ ), 2. Larcopyle buetschlii Dreyer (sample 346-U1425D-1H1-12-14 cm), 3. Lithelius barbatus Motoyama (sample 346-U1425B-47H1-91-93 cm), 4. Larcopyle weddellium Lazarus, Faust and Popova-Goll (sample 346-U1425B-47H1-91-93 cm), 5. Lithelius minor Jørgensen group (sample 346U1425B-29H1-81-83 cm), 6-7. Spongodiscidae juvenile (sample 346-U1425B-23H5-129-131 cm), 8. Spongopyle osculosa Dreyer (sample 346U1425B-23H5-129-131 cm), 9. Stylodictya tenuispina Jørgensen group, 10. Phorticium spp. (sample 346-U1425B-6H5-11-13 cm), 11-12. Tetrapyle spp. (Fig. 11: sample 346-U1425B-6H5-11-13 cm, Fig. 12: sample 346-U1425B-34H4-46-48 cm), 13. Lychnocanoma magnacornuta Sakai (sample 346-U1425B-51HCC-24-29 cm), 14. Lipmanella redondoensis (Campbell and Clark) (sample 346-U1425D-29H1-45-47 cm), 15. Peripyramis circumtexta Haeckel group (sample 346-U1425B-47H1-91-93 cm), 16. Cornutella profunda Ehrenberg group (sample 346-U1425B-47H1-91-93 cm), 17-18. Stichocorys peregrina /delmontensis group (sample 346-U1425B-34H4-46-48 cm), 19-20. Siphocampe arachnea/lineata (Ehrenberg) group (sample 346-U1425B-21H6-19-21 cm), 21-22. Lithomelissa setosa Jørgensen (sample 346-U1425B-5H6-81-83 cm), 23-24. Amphimelissa setosa (Cleve) (sample 346-U1425B-3H4-51-53 cm), 25. Pseudodictyophimus gracilipes (Bailey) (sample 346-U1425B-5H6-81-83 cm), 26. Lychnocanoma parrallelipes Motoyama (sample 346-U1425B-34H4-46-48 cm), 27. Cycladophora nakasekoi Motoyama (sample 346-U1425B-47H1-91-93 cm), 28. Cycladophora sphaeris (Popofsky) (sample 346-U1425B-21H6-19-21 cm), 29. Cycladophora davisiana (Ehrenberg) (sample 346-U1425B-6H5-11-13 cm), 30. Carpocanistrum papillosum (Ehrenberg) group (sample 346-U1425B-34H4-46-48 cm), 31. Ceratospyris borealis Bailey (sample 346-U1425B-3H5-12-14 cm)

assemblage at the site. Six species or species groups dominated at Site U1425: Amphimelissa setosa (Cleve), Cycladophora davisiana (Ehrenberg), Ceratospyris borealis Bailey, Siphocampe lineata/arachnea sensu Nigrini (1977), Cycladophora nakasekoi Motoyama, and the Lithelius barbatus Motoyama group (Figs. 3 and 4). Changes in their relative abundances are described below with their inferred paleoceanographic conditions.

The species $A$. setosa is an extant species inhabiting subsurface water depths $(50-300 \mathrm{~m})$ in the Arctic Ocean
(Bjørklund et al. 2015; Ikenoue et al. 2016; Itaki 2003; Matul and Abelmann 2005). However, this species disappeared from the subarctic North Pacific between marine isotope stages (MISs) 4 and 5 (0.07-0.05 Ma) (e.g., Matul and Abelmann 2005; Ikenoue et al. 2016; Matul et al. 2002; Matsuzaki and Suzuki 2018). In addition, the first occurrence of this species is recorded at $1.48 \mathrm{Ma}$ in the Northeast Pacific (Matsuzaki and Suzuki 2018). In the Japan Sea, this species occurred between 1.47 and $0.05 \mathrm{Ma}$, although our sampling resolution is relatively 
Table 1 Samples used, biostratigraphic tie points, and related sedimentation rates at IODP Site U1425 (cm/ky)

Exp Site Hole Core Type Sect ANW Offset CCSF-A (m) CCSF-D_Patched Age (Ma) Sedimentation Comments

(cm) Mid (Tada et al. (Irino et al. 2018) rates (cm/ky)

\begin{tabular}{lllllllllll} 
2015a) \\
\hline 346 & U1425 & D & 1 & H & 1 & W & 13 & 0.130 & 0.13 & 0.002
\end{tabular}

$\begin{array}{lllllllllll}346 & \text { U1425 } & \mathrm{D} & 1 & \mathrm{H} & 1 & \mathrm{~W} & 103 & 1.030 & 1.03 & 0.017\end{array}$

$\begin{array}{lllllllllll}346 & \text { U1425 } & \mathrm{D} & 1 & \mathrm{H} & 3 & \mathrm{~W} & 3 & 3.030 & 3.03 & 0.051\end{array}$

Tie point of Tada et al. (2018) $\quad 6.22 \quad 0.105$

$105 \quad 5.924 \quad$ We used the Tephra \& Pmag age of Dark layer No. 1-6 (Additional file 3)

\begin{abstract}
$346 \quad U 1425$ B $\quad 1 \quad H$
346 U1425 D 2

346 U1425 D $2 \quad H$

Tie point of Tada et al. (2018)
\end{abstract}

Tie point of Tada et al. (2018)

$346 \cup 1425$ B $2 \quad H$

Tie point of Tada et al. (2018)

Tie point of Tada et al. (2018)

346 U1425 B $3 \quad$

Tie point of Tada et al. (2018)

$346 \cup 1425$ B $\quad 3 \quad$ H

Tie point of Tada et al. (2018)

$\begin{array}{llll}346 & \text { U1425 } & \mathrm{D} & 4\end{array}$

Tie point of Tada et al. (2018)

346 U1425 D $4 \quad H$

Tie point of Tada et al. (2018)

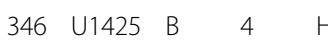

346 U1425 D $5 \quad H$

Tie point of Tada et al. (2018)

Tie point of Tada et al. (2018)

6.992
8.005
10.005

6.99

8.00

10.00

10.55

14.13

16.01

17.66

21.71

22.01

24.90

25.46

27.01

29.18

30.01

33.0

33.21

35.81

38.16

39.23

0.937

40.21

41.75

1.002

43.21

44.97

0.121

0.143

0.185

0.197

0.292

0.342

0.386

0.483

0.493

0.589

0.608

0.647

0.700

0.720

0.790

0.795

0.855

0.909

0.962

1.050

1.109
4.346

4.026

4.251

We used the Tephra \& Pmag age of Dark layer No. 9-1 (Additional file 3)

We used the Tephra \& Pmag age of Dark layer No. 11-1 (Additional file 3)
We used the Tephra \& Pmag age of Dark layer No. 2-5 (Addional file 3) age of Dark layer No. 3-2 (Additional file 3)

We used the Tephra \& Pmag age of Dark layer No. 4-2 (Additional file 3)

We used the Tephra \& Pmag age of Dark layer No. 5-3 (Additional file 3)

We used the Tephra \& Pmag age of Dark layer No. 6-3 (Additional file 3)

We used the Tephra \& Pmag age of Dark layer No. 7-2 (Additional file 3)
We used the Tephra \& Pmag age of Dark layer No. 13-3 (Additional file 3)

We used the Tephra \& Pmag age of Dark layer No. 15-2 (Additional file 3) 
Table 1 Samples used, biostratigraphic tie points, and related sedimentation rates at IODP Site U1425 (cm/ky) (Continued) \begin{tabular}{lllllll}
\hline Exp Site Hole Core Type Sect A/W & $\begin{array}{l}\text { Offset } \\
(\mathrm{cm}) \text { Mid }\end{array}$ & $\begin{array}{c}\text { CCSF-A }(\mathrm{m}) \\
(\text { Tada et al. }\end{array}$ & $\begin{array}{c}\text { CCSF-D_Patched } \\
\text { (Irino et al. 2018) }\end{array}$ & Age (Ma) & $\begin{array}{l}\text { Sedimentation Comments } \\
\text { rates (cm/ky) }\end{array}$
\end{tabular}

(cm) Mid (Tada et al. （Irino et al. 2018) 2015a)

\begin{tabular}{|c|c|c|c|c|c|c|c|c|c|c|c|c|}
\hline 346 & U1425 & $\mathrm{D}$ & 5 & $\mathrm{H}$ & 6 & W & 65 & 45.016 & 45.21 & 1.116 & & \\
\hline 346 & U1425 & B & 5 & $\mathrm{H}$ & 5 & W & 32 & 46.026 & 46.32 & 1.146 & & \\
\hline \multicolumn{9}{|c|}{ Tie point of Tada et al. (2018) } & 47.98 & 1.192 & 3.635 & $\begin{array}{l}\text { We used the Tephra \& Pmag } \\
\text { age of Dark layer No. 17-1 } \\
\text { (Additional file 3) }\end{array}$ \\
\hline 346 & U1425 & B & 5 & $\mathrm{H}$ & 6 & W & 82 & 48.026 & 48.32 & 1.204 & & \\
\hline 346 & U1425 & D & 6 & $\mathrm{H}$ & 3 & W & 134 & 50.990 & 51.28 & 1.306 & & \\
\hline \multicolumn{9}{|c|}{ Tie point of Tada et al. (2018) } & 51.43 & 1.311 & 2.892 & $\begin{array}{l}\text { We used the Tephra \& Pmag } \\
\text { age of Dark layer No. 19-1 } \\
\text { (Additional file 3) }\end{array}$ \\
\hline
\end{tabular}

$\begin{array}{llllllll}346 & \text { U1425 } & \text { B } & 6 & \text { H } & 2 & \text { W } & 62\end{array}$

Tie point of Tada et al. (2018)

$\begin{array}{llllllll}346 & \text { U1425 } & \text { B } & 6 & \text { H } & 3 & \text { W } & 112\end{array}$

$\begin{array}{llllllll}346 & \mathrm{U} 1425 & \mathrm{~B} & 6 & \mathrm{H} & 5 & \mathrm{~W} & 12\end{array}$

$\begin{array}{llllllll}346 & \mathrm{U} 1425 & \mathrm{D} & 7 & \mathrm{H} & 2 & \mathrm{~W} & 82\end{array}$

Tie point of Kamikuri et al. (2017)
55.017

57.017

60.013

64.115
53.31

54.8

55.31

57.31

60.30

64.63

65.013

68.016

69.996

73.006

76.007

79.007

80.005
65.30

68.31

70.29

73.30

76.30

79.30

80.62
1.391

1.455

1.468

1.517

1.592

1.700

1.750

1.972

2.119

2.342

2.564

2.787

2.885 (Additional file 3)

2.344 We used the Tephra \& Pmag age of Dark layer No. 21-2 (Additional file 3)

Tie point of Kamikuri et al. (2017)

$\begin{array}{llllllll}346 & \text { U1425 } & \text { B } & 8 & \text { H } & 7 & \text { W } & 39 \\ 346 & \text { U1425 } & \text { B } & 9 & \text { H } & 3 & \text { W } & 5 \\ 346 & \text { U1425 } & \text { B } & 9 & \text { H } & 4 & \text { W } & 55 \\ 346 & \text { U1425 } & \text { D } & 10 & \text { H } & 2 & \text { W } & 65 \\ 346 & \text { U1425 } & \text { D } & 10 & \text { H } & 4 & \text { W } & 65 \\ 346 & \text { U1425 } & \text { D } & 10 & \text { H } & 6 & \text { W } & 66 \\ 346 & \text { U1425 } & \text { B } & 10 & \text { H } & 5 & \text { W } & 94 \\ 346 & \text { U1425 } & \text { D } & 11 & \text { H } & 3 & \text { W } & 133 \\ 346 & \text { U1425 } & \text { D } & 11 & \text { H } & 5 & \text { W } & 133 \\ 346 & \text { U1425 } & \text { B } & 11 & \text { H } & 5 & \text { W } & 62 \\ 346 & \text { U1425 } & \text { D } & 12 & \text { H } & 3 & \text { W } & 75 \\ 346 & \text { U1425 } & \text { D } & 13 & \text { X } & 2 & \text { W } & 71 \\ 346 & \text { U1425 } & \text { B } & 14 & \text { H } & 3 & \text { W } & 59\end{array}$

81.017

85.002

87.002

88.007

91.007

94.017

96.013

99.003

102.003

106.008

109.005

112.011

115.014
81.57

85.55

87.55

88.56

91.56

93.75

95.74

98.73

101.73

105.74

108.73

111.86

114.86
2.905

2.992

3.035

3.057

3.122

3.170

3.213

3.278

3.343

3.430

3.495

3.563

3.628 
Table 1 Samples used, biostratigraphic tie points, and related sedimentation rates at IODP Site U1425 (cm/ky) (Continued)

Exp Site Hole Core Type Sect ANW Offset CCSF-A (m) CCSF-D_Patched Age (Ma) Sedimentation Comments

(cm) Mid (Tada et al. （Irino et al. 2018) 2015a)

\begin{tabular}{lllllllllll}
\hline 346 & U1425 & B & 14 & H & 5 & W & 56 & 118.014 & 117.86 & 3.694 \\
346 & U1425 & B & 14 & H & 6 & W & 106 & 120.014 & 119.86 & 3.737 \\
346 & U1425 & D & 14 & H & 2 & W & 59 & 122.009 & 121.86 & 3.780 \\
346 & U1425 & D & 14 & H & 3 & W & 109 & 124.009 & 123.86 & 3.824 \\
346 & U1425 & B & 17 & H & 2 & W & 124 & 125.003 & 124.85 & 3.845 \\
346 & U1425 & D & 15 & H & 3 & W & 23 & 127.011 & 126.86 & 3.889 \\
346 & U1425 & D & 15 & H & 4 & W & 73 & 129.011 & 128.86 & 3.932 \\
346 & U1425 & D & 15 & H & 6 & W & 73 & 132.011 & 131.86 & 3.997 \\
Tie point of Kamikuri et al. (2017) & & & & 134.43 & 134.28 & 4.050
\end{tabular}

4.606

We used the Last Occurrence of D. bullatus (Radiolaria), which occurred at the mid depth of $134.43 \mathrm{~m}$ CCSF-A. This bioevent has an error margin of $0.25 \mathrm{Ma}$.

$\begin{array}{lllllllllll}346 & \text { U1425 } & \text { D } & 16 & \text { H } & 2 & \text { W } & 4 & 136.013 & 135.86 & 4.089 \\ 346 & \text { U1425 } & \text { D } & 16 & \text { H } & 4 & \text { W } & 2 & 139.013 & 138.86 & 4.164 \\ 346 & \text { U1425 } & \text { D } & 16 & \text { H } & 6 & \text { W } & 2 & 142.013 & 141.86 & 4.238 \\ 346 & \text { U1425 } & \text { B } & 21 & \text { H } & 2 & \text { W } & 21 & 144.030 & 143.88 & 4.289 \\ 346 & \text { U1425 } & \text { B } & 21 & \text { H } & 4 & \text { W } & 20 & 147.020 & 146.87 & 4.363 \\ \text { Tie point of Kamikuri et al. (2017) } & & & & 148.51 & 148.36 & 4.400\end{array}$

4.164

4.238

4.289

4.400

4.023

We used the First Occurrence of D. bullatus (Radiolaria), which occurred at the mid depth of $148.51 \mathrm{~m}$ CCSF-A. This bioevent has an error margin of $0.1 \mathrm{Ma}$.

$\begin{array}{llllllll}346 & \text { U1425 } & \text { B } & 21 & \text { H } & 6 & \text { W } & 20 \\ 346 & \text { U1425 } & \text { D } & 18 & \text { H } & 5 & \text { W } & 7 \\ 346 & \text { U1425 } & \text { D } & 18 & \text { H } & 6 & \text { W } & 60 \\ 346 & \text { U1425 } & \text { B } & 22 & \text { H } & 3 & \text { W } & 111 \\ 346 & \text { U1425 } & \text { B } & 22 & \text { H } & 5 & \text { W } & 11\end{array}$

150.020

149.87

4.480

151.996

151.84

4.584

154.026

153.87

4.691

156.990

156.84

4.848

158.990

158.84

4.954

Tie point of Kamikuri et al. (2017)
160.66
5.054

5.111

5.167

5.224

5.262

5.299

5.337

5.375

5.413

5.470

5.507
We used the Last Occurrence of $L$. redondoensis (Radiolaria), which occurred at the mid depth of 160,815 m CCSF-A. This bioevent has an error margin of $0.05 \mathrm{Ma}$. 
Table 1 Samples used, biostratigraphic tie points, and related sedimentation rates at IODP Site U1425 (cm/ky) (Continued)

Exp Site Hole Core Type Sect ANW Offset CCSF-A (m) CCSF-D_Patched Age (Ma) Sedimentation Comments

(cm) Mid (Tada et al. （Irino et al. 2018) 2015a)

\begin{tabular}{|c|c|c|c|c|c|c|c|c|c|c|}
\hline 346 & U1425 & B & 25 & $\mathrm{H}$ & 4 & W & 92 & 188.011 & 187.86 & 5.564 \\
\hline 346 & U1425 & D & 22 & $\mathrm{H}$ & 5 & W & 62 & 193.011 & 192.86 & 5.659 \\
\hline 346 & U1425 & D & 22 & $\mathrm{H}$ & 6 & W & 112 & 195.011 & 194.86 & 5.696 \\
\hline 346 & U1425 & D & 23 & $\mathrm{H}$ & 2 & W & 77 & 198.007 & 197.86 & 5.753 \\
\hline 346 & U1425 & D & 23 & $\mathrm{H}$ & 4 & W & 77 & 201.007 & 200.86 & 5.810 \\
\hline 346 & U1425 & D & 23 & $\mathrm{H}$ & 6 & W & 77 & 204.007 & 203.86 & 5.867 \\
\hline 346 & U1425 & $B$ & 27 & $\mathrm{H}$ & 4 & W & 28 & 207.014 & 206.86 & 5.923 \\
\hline 346 & U1425 & B & 27 & $\mathrm{H}$ & 6 & W & 27 & 210.004 & 209.85 & 5.980 \\
\hline 346 & U1425 & D & 24 & $\mathrm{H}$ & 5 & W & 6 & 212.008 & 211.86 & 6.018 \\
\hline 346 & U1425 & D & 24 & $\mathrm{H}$ & 6 & W & 56 & 214.008 & 213.86 & 6.056 \\
\hline 346 & U1425 & B & 28 & $\mathrm{H}$ & 3 & W & 148 & 217.010 & 216.86 & 6.112 \\
\hline \multicolumn{8}{|c|}{ Tie point of Kamikuri et al. (2017) } & 219 & 218.85 & 6.15 \\
\hline
\end{tabular}

\begin{tabular}{lllllllllll}
346 & U1425 & B & 29 & H & 1 & W & 82 & 222.021 & 221.99 & 6.267 \\
346 & U1425 & B & 29 & H & 3 & W & 82 & 225.021 & 224.99 & 6.378 \\
346 & U1425 & B & 29 & H & 5 & W & 83 & 228.031 & 228.00 & 6.489 \\
346 & U1425 & D & 27 & H & 1 & W & 123 & 231.023 & 231.00 & 6.600 \\
346 & U1425 & B & 30 & H & 3 & W & 32 & 233.012 & 234.23 & 6.720 \\
346 & U1425 & B & 30 & H & 5 & W & 32 & 236.012 & 237.23 & 6.831 \\
\multicolumn{7}{l}{ Tie point of Kamikuri et al. (2017) }
\end{tabular}

$\begin{array}{llllllll}346 & \text { U1425 } & \text { D } & 28 & \text { H } & 4 & \text { W } & 90 \\ 346 & \text { U1425 } & \text { D } & 28 & \text { H } & 6 & \text { W } & 90 \\ 346 & \text { U1425 } & \text { D } & 29 & \text { H } & 1 & \text { W } & 46 \\ 346 & \text { U1425 } & \text { D } & 29 & \text { H } & 2 & \text { W } & 96 \\ 346 & \text { U1425 } & \text { B } & 33 & \text { H } & 3 & \text { W } & 40 \\ 346 & \text { U1425 } & \text { B } & 33 & \text { H } & 4 & \text { W } & 90 \\ 346 & \text { U1425 } & \text { B } & 33 & \text { H } & 5 & \text { W } & 139 \\ 346 & \text { U1425 } & \text { B } & 33 & \text { H } & 6 & \text { W } & 91 \\ 346 & \text { U1425 } & \text { D } & 30 & \text { H } & 5 & \text { W } & 76\end{array}$

Tie point of Kamikuri et al. (2017)

$\begin{array}{lll}238.009 & 239.23 & 6.903 \\ 241.009 & 242.23 & 6.968 \\ 243.011 & 244.23 & 7.012 \\ 245.011 & 246.23 & 7.055 \\ 247.002 & 248.22 & 7.099 \\ 249.002 & 250.22 & 7.142 \\ 250.992 & 252.21 & 7.186 \\ 252.012 & 253.23 & 7.208 \\ 255.009 & 256.23 & 7.273 \\ 255.58 & 257.46 & 7.300\end{array}$

6.903

6.968

7.012

7.099

7.142

7.186

208

7.300 rates $(\mathrm{cm} / \mathrm{ky})$

\subsection{9}

.753

810

5.867

5923

6.018

56

$150 \quad 5.290 \quad$ We used the Last Occurrence of L. parallelipes (Radiolaria), which occurred at the mid depth of $219.00 \mathrm{~m}$ CCSF-A. This bioevent has an error margin of $0.25 \mathrm{Ma}$.

2.699 We used the Rapid Increase of L. barbatus (Radiolaria), which occurred at the mid depth of $237.66 \mathrm{~m}$ CCSF-A. This bioevent has an error margin of $0.10 \mathrm{Ma}$.

$\begin{array}{lllllllllll}346 & \text { U1425 } & \text { B } & 34 & \text { H } & 4 & \text { W } & 47 & 258.010 & 259.23 & 7.342 \\ 346 & \text { U1425 } & \text { B } & 34 & \text { H } & 5 & \text { W } & 97 & 260.010 & 262.23 & 7.413 \\ 346 & \text { U1425 } & \text { D } & 31 & \text { H } & 2 & \text { W } & 118 & 261.012 & 265.23 & 7.484\end{array}$


Table 1 Samples used, biostratigraphic tie points, and related sedimentation rates at IODP Site U1425 (cm/ky) (Continued)

\begin{tabular}{|c|c|c|c|c|c|c|c|c|c|c|c|c|}
\hline Exp & Site & Hole & Core & Type & Sect & A/W & $\begin{array}{l}\text { Offset } \\
(\mathrm{cm}) \mathrm{Mid}\end{array}$ & $\begin{array}{l}\text { CCSF-A (m) } \\
\text { (Tada et al. } \\
\text { 2015a) }\end{array}$ & $\begin{array}{l}\text { CCSF-D_Patched } \\
\text { (Irino et al. 2018) }\end{array}$ & Age (Ma) & $\begin{array}{l}\text { Sedimentation } \\
\text { rates }(\mathrm{cm} / \mathrm{ky})\end{array}$ & Comments \\
\hline 346 & U1425 & $D$ & 31 & $\mathrm{H}$ & 4 & W & 118 & 264.012 & 267.23 & 7.531 & & \\
\hline 346 & U1425 & B & 35 & $\mathrm{H}$ & 3 & W & 48 & 266.008 & 268.23 & 7.555 & & \\
\hline 346 & U1425 & B & 35 & $\mathrm{H}$ & 3 & W & 148 & 267.008 & 270.23 & 7.602 & & \\
\hline 346 & U1425 & B & 35 & $\mathrm{H}$ & 5 & W & 48 & 269.008 & 274.23 & 7.697 & & \\
\hline 346 & U1425 & D & 32 & $\mathrm{H}$ & 4 & W & 6 & 273.007 & 275.23 & 7.720 & & \\
\hline 346 & U1425 & $D$ & 32 & $\mathrm{H}$ & 4 & W & 106 & 274.007 & 277.23 & 7.768 & & \\
\hline 346 & U1425 & $D$ & 32 & $\mathrm{H}$ & 6 & W & 6 & 276.007 & 280.23 & 7.839 & & \\
\hline 346 & U1425 & B & 37 & $\mathrm{H}$ & 1 & W & 129 & 279.011 & 281.24 & 7.863 & & \\
\hline 346 & U1425 & B & 37 & $\mathrm{H}$ & 2 & W & 80 & 280.021 & 281.26 & 7.863 & & \\
\hline 346 & U1425 & D & 33 & $\mathrm{H}$ & 4 & W & 3 & 282.014 & 283.23 & 7.910 & & \\
\hline 346 & U1425 & B & 38 & $\mathrm{H}$ & 1 & W & 102 & 283.006 & 284.22 & 7.933 & & \\
\hline 346 & U1425 & $D$ & 35 & $\mathrm{H}$ & 2 & W & 48 & 287.003 & 288.22 & 8.028 & & \\
\hline 346 & U1425 & B & 39 & $\mathrm{H}$ & 2 & W & 126 & 290.011 & 291.23 & 8.099 & & \\
\hline 346 & U1425 & B & 39 & $\mathrm{H}$ & 4 & W & 61 & 291.991 & 293.21 & 8.146 & & \\
\hline 346 & U1425 & B & 40 & $x$ & 2 & W & 59 & 293.998 & 294.22 & 8.170 & & \\
\hline 346 & U1425 & B & 40 & $x$ & 3 & W & 10 & 295.008 & 296.22 & 8.217 & & \\
\hline 346 & U1425 & $D$ & 37 & $\mathrm{H}$ & 1 & W & 144 & 297.010 & 298.36 & 8.268 & & \\
\hline 346 & U1425 & $D$ & 37 & $\mathrm{H}$ & 2 & W & 94 & 298.010 & 299.36 & 8.292 & & \\
\hline 346 & U1425 & $D$ & 37 & $\mathrm{H}$ & 4 & W & 95 & 300.010 & 301.36 & 8.339 & & \\
\hline 346 & U1425 & $D$ & 37 & $\mathrm{H}$ & 5 & W & 45 & 301.010 & 302.36 & 8.363 & & \\
\hline 346 & U1425 & B & 42 & $\mathrm{H}$ & 3 & W & 67 & 306.015 & 307.36 & 8.481 & & \\
\hline 346 & U1425 & D & 38 & $\mathrm{H}$ & 2 & W & 127 & 307.006 & 308.35 & 8.505 & & \\
\hline 346 & U1425 & $D$ & 39 & $\mathrm{H}$ & 1 & W & 57 & 310.003 & 311.35 & 8.576 & & \\
\hline 346 & U1425 & $D$ & 39 & $\mathrm{H}$ & 2 & W & 107 & 312.003 & 313.35 & 8.623 & & \\
\hline 346 & U1425 & $D$ & 39 & $\mathrm{H}$ & 3 & W & 57 & 313.003 & 314.35 & 8.647 & & \\
\hline 346 & U1425 & B & 44 & $\mathrm{H}$ & 2 & W & 77 & 314.012 & 315.36 & 8.670 & & \\
\hline 346 & U1425 & B & 44 & $\mathrm{H}$ & 4 & W & 5 & 316.012 & 317.36 & 8.718 & & \\
\hline 346 & U1425 & D & 40 & $\mathrm{H}$ & 2 & W & 60 & 318.013 & 319.36 & 8.765 & & \\
\hline 346 & U1425 & D & 40 & $\mathrm{H}$ & 3 & W & 10 & 319.013 & 320.36 & 8.789 & & \\
\hline 346 & U1425 & $D$ & 40 & $\mathrm{H}$ & 3 & W & 110 & 320.013 & 321.36 & 8.812 & & \\
\hline 346 & U1425 & B & 46 & $\mathrm{H}$ & 1 & W & 53 & 321.027 & 321.85 & 8.824 & & \\
\hline 346 & U1425 & $D$ & 41 & $\mathrm{H}$ & 2 & W & 109 & 323.018 & 323.85 & 8.871 & & \\
\hline 346 & U1425 & $D$ & 41 & $\mathrm{H}$ & 4 & W & 37 & 325.008 & 325.84 & 8.918 & & \\
\hline 346 & U1425 & B & 47 & $\mathrm{H}$ & 1 & W & 92 & 326.016 & 326.84 & 8.942 & & \\
\hline 346 & U1425 & B & 47 & $\mathrm{H}$ & 2 & W & 42 & 327.016 & 327.84 & 8.966 & & \\
\hline 346 & U1425 & B & 47 & $\mathrm{H}$ & 2 & W & 143 & 328.026 & 328.85 & 8.990 & & \\
\hline 346 & U1425 & B & 47 & $\mathrm{H}$ & 3 & W & 92 & 329.016 & 329.84 & 9.013 & & \\
\hline 346 & U1425 & $D$ & 43 & $\mathrm{H}$ & 2 & W & 28 & 331.016 & 331.84 & 9.061 & & \\
\hline \multicolumn{8}{|c|}{ Tie point of Kamikuri et al. (2017) } & 332.68 & 333.51 & 9.100 & 4.225 & $\begin{array}{l}\text { We used the First Occurrence } \\
\text { of L. barbatus (Radiolaria), } \\
\text { which occurred at the } \\
\text { mid depth of } 255.58 \mathrm{~m} \\
\text { CCSF-A. This bioevent has } \\
\text { no an error margin. }\end{array}$ \\
\hline
\end{tabular}


Table 1 Samples used, biostratigraphic tie points, and related sedimentation rates at IODP Site U1425 (cm/ky) (Continued)

\begin{tabular}{|c|c|c|c|c|c|c|c|c|c|c|c|c|}
\hline Exp & Site & Hole & Core & Type & Sect & A/W & $\begin{array}{l}\text { Offset } \\
(\mathrm{cm}) \mathrm{Mid}\end{array}$ & $\begin{array}{l}\text { CCSF-A (m) } \\
\text { (Tada et al. } \\
\text { 2015a) }\end{array}$ & $\begin{array}{l}\text { CCSF-D_Patched } \\
\text { (Irino et al. 2018) }\end{array}$ & Age (Ma) & $\begin{array}{l}\text { Sedimentation } \\
\text { rates }(\mathrm{cm} / \mathrm{ky})\end{array}$ & Comments \\
\hline 346 & U1425 & $\mathrm{D}$ & 43 & $\mathrm{H}$ & 3 & W & 78 & 333.016 & 333.84 & 9.106 & & \\
\hline 346 & U1425 & D & 43 & $\mathrm{H}$ & 4 & W & 28 & 334.016 & 334.84 & 9.123 & & \\
\hline 346 & U1425 & D & 43 & $\mathrm{H}$ & 4 & W & 128 & 335.016 & 335.84 & 9.140 & & \\
\hline 346 & U1425 & D & 43 & $\mathrm{H}$ & 6 & W & 28 & 337.016 & 337.84 & 9.173 & & \\
\hline 346 & U1425 & B & 49 & $\mathrm{H}$ & 2 & W & 30 & 338.016 & 338.84 & 9.190 & & \\
\hline 346 & U1425 & B & 49 & H & 2 & W & 130 & 339.016 & 339.84 & 9.207 & & \\
\hline 346 & U1425 & D & 44 & $\mathrm{H}$ & 2 & W & 31 & 341.014 & 341.84 & 9.241 & & \\
\hline 346 & U1425 & B & 50 & $\mathrm{H}$ & 2 & W & 67 & 343.994 & 344.82 & 9.291 & & \\
\hline 346 & U1425 & B & 50 & $\mathrm{H}$ & 3 & W & 118 & 346.004 & 346.83 & 9.325 & & \\
\hline 346 & U1425 & D & 45 & $\mathrm{H}$ & 2 & W & 41 & 347.010 & 347.84 & 9.342 & & \\
\hline 346 & U1425 & D & 45 & $\mathrm{H}$ & 2 & W & 141 & 348.010 & 348.84 & 9.359 & & \\
\hline 346 & U1425 & D & 46 & $\mathrm{H}$ & 1 & W & 76 & 349.73 & 350.56 & 9.393 & & \\
\hline 346 & U1425 & D & 46 & H & 2 & W & 76 & 351.23 & 352.06 & 9.423 & & \\
\hline 346 & U1425 & D & 46 & $\mathrm{H}$ & 4 & W & 4 & 353.23 & 354.06 & 9.462 & & \\
\hline 346 & U1425 & D & 48 & H & 1 & W & 26 & 358.63 & 359.46 & 9.568 & & \\
\hline \multicolumn{8}{|c|}{ Tie point of Kamikuri et al. (2017) } & 382.925 & 383.75 & 9.950 & 5.911 & $\begin{array}{l}\text { We used the First Occurrence } \\
\text { of C. nakasekoi (Radiolaria), } \\
\text { which occurred at the } \\
\text { mid depth of } 382.925 \mathrm{~m} \\
\text { CCSF-A. This bioevent has an } \\
\text { error margin of } 0.25 \mathrm{Ma} \text {. }\end{array}$ \\
\hline
\end{tabular}

low for the Pleistocene (Fig. 3). During this time interval (1.47-0.05 Ma), the mean of $A$. setosa relative abundance is $10.6 \%(\mathrm{SD}=18.3 ; N=22)$. The highest abundance of this species is $80.55 \%$ recorded at $0.34 \mathrm{Ma}$ (Fig. 3 and Additional file 1).

The species $C$. davisiana and $C$. borealis are both subarctic, abundant in areas influenced by the Oyashio Current and in the Sea of Okhotsk (e.g., Matsuzaki and Itaki 2017; Matsuzaki et al. 2014a, 2014b; Okazaki et al. 2004). In the Japan Sea, C. davisiana is related to the Japan Sea deep water formed by cooling of the TWC, that is, the Japan Sea Proper Water (Itaki 2003; Itaki et al. 2007), while C. borealis prefers cold surface and subsurface water (50200 m) (Itaki 2003; Itaki et al. 2007; Okazaki et al. 2004). C. davisiana is recorded at Site U1425 between 3.21 and $0 \mathrm{Ma}$, with a mean relative abundance of $6.4 \%(\mathrm{SD}=13.1 ; N=37)$, while $C$. borealis is recorded between 3.56 and $0 \mathrm{Ma}$ with a mean relative abundance of $8.4 \% \quad(\mathrm{SD}=15.2 ; N=42) \quad$ (Fig. 3 and Additional file 1).

The species group $S$. lineata/arachnea is an extant species inhabiting water between 300 and $1000 \mathrm{~m}$ deep in the North Pacific (Kling and Boltovskoy 1995). Higher abundances of this species are recorded in the arctic to subarctic areas of the North Pacific (e.g., Boltovskoy and Correa 2016; Kling and Boltovskoy 1995; Takahashi 1991). Additionally, Boltovskoy and Correa (2016) showed that this species is restricted to the Northern Hemisphere high latitudes. Therefore, we assume that this species group is related to the intermediate water of the Northern Hemisphere high latitudes. At Site U1425, this species is encountered intermittently between 9.5 and $0 \mathrm{Ma}$, with a mean relative abundance of $4.3 \%(\mathrm{SD}=11.4 ; N=154)$ (Additional file 1$)$. This species dominates the radiolarian assemblage (>30\%) between 4.9 and $4.0 \mathrm{Ma}$ (Fig. 4 and Additional file 1).

The L. barbatus group is an extinct group (e.g., Motoyama 1996; Kamikuri et al. 2004, 2007) and was described as having relatively wide variation of morphological forms by Motoyama (1996). This species group has been encountered in areas related to the subarctic gyre of the North Pacific (e.g., Motoyama 1996; Kamikuri et al. 2004, 2007), whereas this species group is absent in areas influenced by the subtropical gyre (Kamikuri 2017; Kamikuri et al. 2009). Therefore, we assumed that this species is related to water masses of transitional to subarctic areas. At Site U1425, this species is encountered between 7.55 and $5.51 \mathrm{Ma}$, with a 
mean relative abundance of $11.6 \%(\mathrm{SD}=12.9 ; N=32)$ (Fig. 4 and Additional file 1). This species' relative abundance is higher than the mean value between 7.01 and $5.75 \mathrm{Ma}$, and it dominates the radiolarian assemblage between 6.11 and $6.02 \mathrm{Ma}$ (>30\%) (Fig. 4).

Finally, C. nakasekoi is an extinct species endemic to the Japan Sea (Motoyama 1996; Kamikuri et al. 2004, 2007), but its related ecology, such as depth of its habitat and preferred climatic condition, is unknown. This species is recorded between 9.57 and $7.41 \mathrm{Ma}$ at Site U1425, with a mean relative abundance of $20.0 \%$ $(\mathrm{SD}=12.7 ; N=55)$ (Fig. 4 and Additional file 1). The relative abundance of $C$. nakasekoi fluctuates between high $(>30 \%)$ and low relative abundances $(<10 \%)$ (Fig. 4). Interestingly, the sediments collected at Site U1425 spanning the time interval between 9.57 and $7.41 \mathrm{Ma}$ correspond to lithological subunit IIIA, composed of alternating dark gray diatom ooze and heavily bioturbated clayey diatomaceous ooze (Tada et al. 2015a).

\section{Relative abundance of abundant species}

We have defined a species or species group as abundant when its relative abundance is between 10 and $30 \%$ of the total assemblage at least once at the site. At Site U1425, abundant species are Lithomelissa setosa Jørgensen, Spongodiscidae juvenile, Pseudodictyophimus gracilipes (Bailey), Cycladophora sphaeris (Popofsky), Phorticium spp., Tetrapyle spp., Lithelius minor Jørgensen group, Stylodictya tenuispina Jørgensen group, Stichocorys delmontensis (Campbell and Clark), Stylochlanydium venustum/bensoni group, Larcopyle polyacantha group (Campbell and Clark), and Stichocorys peregrina (Riedel) (Figs. 3 and 4). The last two species are grouped as Stichocorys delmontensis/peregrina in Fig. 4.

Species such as L. setosa, Spongodiscidae juvenile, and $P$. gracilipes are well-known extant species related to subarctic areas. Based on previous studies, we know that $L$. setosa is related to the shallow water of subarctic to arctic areas of the Northern Hemisphere (Atlantic and Pacific oceans) (Boltovskoy and Correa 2016; Matsuzaki and Itaki 2017; Okazaki et al. 2004). At Site U1425, this species was recorded between 3.69 and $0 \mathrm{Ma}$, and its mean relative abundance is $1.7 \%(\mathrm{SD}=3.3 ; N=44)$ (Fig. 3 and Additional file 1). P. gracilipes is related to the upper intermediate water of areas influenced by the Oyashio Current (300-500 m). Young forms of Spongodiscidae, whose taxonomy is still unsettled, are often named as Spongodiscus resurgens Ehrenberg group (e.g., Itaki 2009; Matsuzaki et al. 2015a). However, whichever name is used, this form is likely related to the shallow subsurface waters of transitional subarctic areas in the
Northwest Pacific (e.g., Kamikuri et al. 2008; Matsuzaki and Itaki 2017; Okazaki et al. 2004). In our study, both the species and species group were found at Site U1425 throughout the period since $9.5 \mathrm{Ma}$. The mean relative abundance of $P$. gracilipes is $1.2 \%(\mathrm{SD}=2.2 ; N=154)$, while the mean relative abundance of Spongodiscidae juvenile is $5.2 \%(\mathrm{SD}=6.2 ; N=154)$ (Fig. 3 and Additional file 1). For P. gracilipes, relative abundance higher than the mean value is recorded intermittently between 3.85 and $2.79 \mathrm{Ma}, 1.75$ and $1.12 \mathrm{Ma}$, and 0.65 and $0.49 \mathrm{Ma}$ (Fig. 3 and Additional file 1). For juvenile Spongodiscidae, progressive increases in its relative abundance are recorded since $7.34 \mathrm{Ma}$, where its relative abundance exceeds the mean value for the first time (12.47\%). From 4.2 and $0.49 \mathrm{Ma}$, relative abundance higher than $11 \%$ is constantly recorded (Fig. 3 and Additional file 1).

C. sphaeris is an extinct species, but it likely inhabited water of transitional-subarctic to subarctic areas of the North Pacific (e.g., Kamikuri et al. 2007; Motoyama 1996; Oseki and Suzuki 2009). In our study, this species occurred between 7.93 and $3.06 \mathrm{Ma}$ at Site U1425 and the mean relative abundance is $9.3 \%(\mathrm{SD}=6.9 ; N=79)$ (Fig. 3 and Additional file 1). Highest abundance of this species was recorded in the late Pliocene at 3.34 Ma (36.62\%) (i.e., Fig. 3 and Additional file 1).

Phorticium spp. and Tetrapyle spp. are extant species groups, both known as a marker of warm shallow water in the North Pacific (e.g., Lombari and Boden 1985; Itaki et al. 2010; Kamikuri et al. 2008; Matsuzaki et al 2014b; Matsuzaki et al. 2015b; Matsuzaki et al. 2016; Matsuzaki and Itaki 2017). However, the main difference between the two species groups is that while Tetrapyle spp. are restricted to the low-middle latitudes, Phorticium spp. are also common in temperate areas (Kamikuri et al. 2008; Romine 1985). According to Zhang and Suzuki (2017), our Phorticium spp. and Tetrapyle spp. might also include morphotypes from different genera because for the Miocene, the taxonomy of both genera is poorly settled and accurate taxonomic study of both genera is required for this period. However, most of the extant taxa, which potentially belong to these two species groups, bear algal symbionts and inhabit warmer shallow waters (Zhang et al. 2018). Therefore, this taxonomic concern does not affect our paleoceanographic reconstruction. At Site U1425, both species groups are recorded continuously since $9.5 \mathrm{Ma}$, although Tetrapyle spp. seem to be absent from 5.05 to $3.06 \mathrm{Ma}$ (Fig. 4 and Additional file 1). Phorticium spp. have a mean relative abundance of $5.3 \%(\mathrm{SD}=6 ; N=154)$, while Tetrapyle spp. have a mean relative abundance of $1.9 \%(\mathrm{SD}=3.1$; $\mathrm{N}=154$ ) (Fig. 4 and Additional file 1). Tetrapyle spp. have a relative abundance twice as high as the mean value for the periods between 9.57 and 7.06 $\mathrm{Ma}$ and 
Table 2 Samples used, biostratigraphic tie points, and related sedimentation rates at IODP Site U1430 (cm/ky)

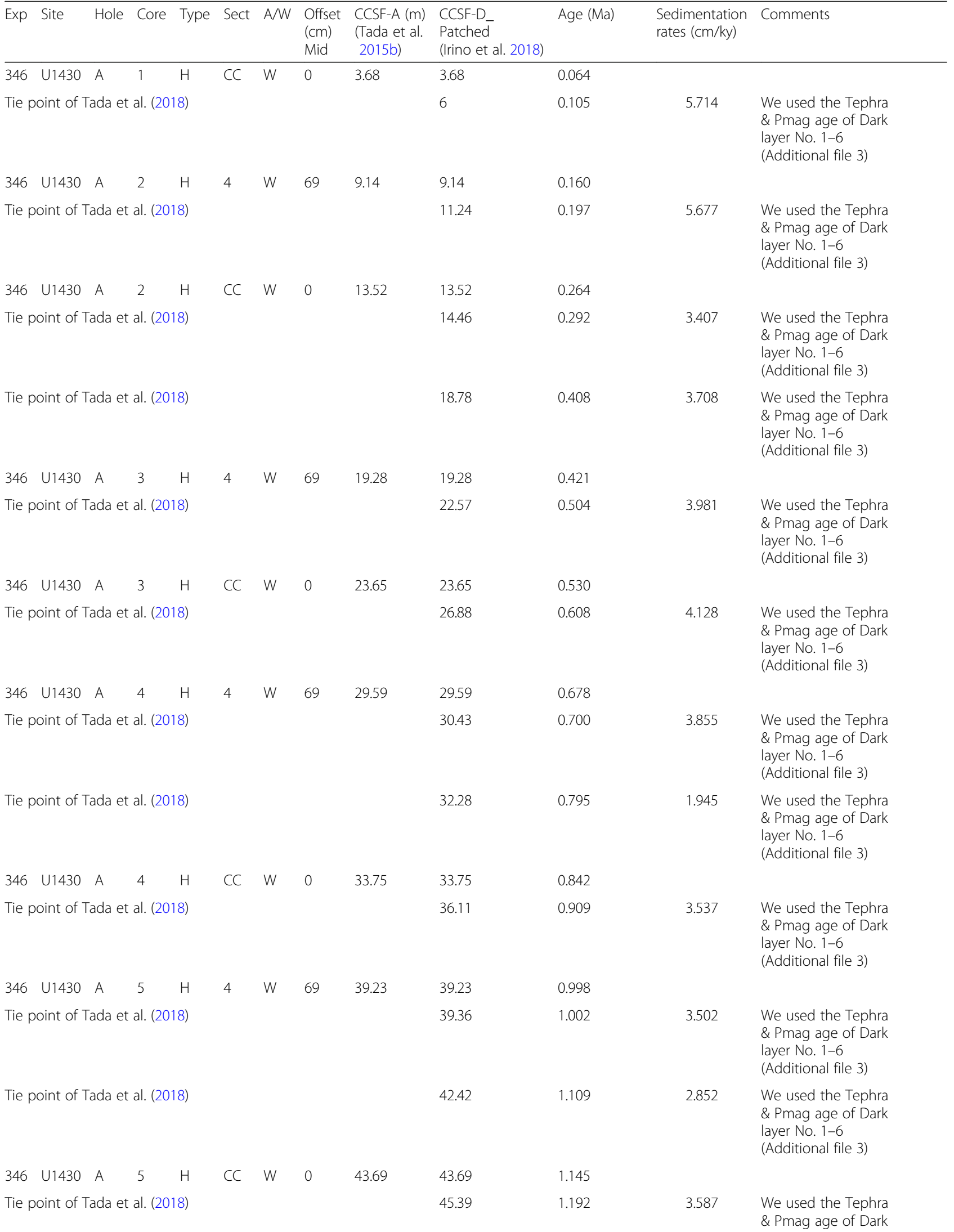


Table 2 Samples used, biostratigraphic tie points, and related sedimentation rates at IODP Site U1430 (cm/ky) (Continued)

\begin{tabular}{|c|c|c|c|c|c|c|c|c|c|c|}
\hline Exp & Site & Hole & Core & Type & Sect & A/W & $\begin{array}{l}\text { Offset } \\
(\mathrm{cm}) \\
\text { Mid }\end{array}$ & $\begin{array}{l}\text { CCSF-A (m) } \\
\text { (Tada et al. } \\
\text { 2015b) }\end{array}$ & $\begin{array}{l}\text { CCSF-D_- } \\
\text { Patched } \\
\text { (Irino et al. 2018) }\end{array}$ & Age \\
\hline \multicolumn{9}{|c|}{ Tie point of Tada et al. (2018) } & 48.23 & \\
\hline 346 & U1430 & A & 6 & $\mathrm{H}$ & 4 & W & 69 & 49.15 & 49.15 & \\
\hline \multicolumn{9}{|c|}{ Tie point of Tada et al. (2018) } & 50.66 & \\
\hline 346 & U1430 & A & 6 & $\mathrm{H}$ & $\mathrm{CC}$ & W & 0 & 53.56 & 53.56 & \\
\hline 346 & U1430 & A & 7 & $\mathrm{H}$ & 4 & W & 69 & 59.2 & 59.2 & \\
\hline \multicolumn{8}{|c|}{ Tie point of Kamikuri et al. (2017) } & 61.41 & 61.41 & \\
\hline
\end{tabular}

$\begin{array}{cl}\begin{array}{l}\text { Sedimentation } \\ \text { rates }(\mathrm{cm} / \mathrm{ky})\end{array} & \text { Comments } \\ & \begin{array}{c}\text { layer No. 1-6 } \\ \text { (Additional file 3) }\end{array} \\ & \text { We used the Tephra } \\ & \text { \& Pmag age of Dark } \\ & \text { layer No. 1-6 } \\ & \text { (Additional file 3) }\end{array}$

.366

.455

.841

592

.885

$0.751 \quad$ We used the Last Occurrence of H. parviakitaense (Radiolaria), which occurred at the mid depth of $61.41 \mathrm{~m}$ CCSF-A. This bioevent has an error margin of $0.015 \mathrm{Ma}$.

3.073

3.551

3.931

3.987

4.050

$1.174 \quad$ We used the First Occurrence of D. bullatus (Radiolaria), which occurred at the mid depth of 75.08 m CCSF-A. This bioevent has an error margin of $0.25 \mathrm{Ma}$.

We defined this depths interval as a hiatus following the findings of Tada et al. (2015b), Kamikuri et al. (2017)

We used the First Occurrence of L. parallelipes (Radiolaria), which occurred at the mid depth of 80.06 m CCSF-A. This bioevent has an error margin of $0.10 \mathrm{Ma}$.

$\begin{array}{lllllllllll}346 & \text { U1430 } & \text { A } & 9 & \text { H } & 5 & \text { W } & 71 & 80.35 & 80.35 & 7.3075186054 \\ 346 & \text { U1430 } & \text { A } & 9 & \text { H } & 6 & \text { W } & 71 & 81.85 & 81.85 & 7.3457488021 \\ 346 & \text { U1430 } & \text { A } & 9 & \text { H } & \text { CC } & \text { W } & 0 & 83.22 & 83.22 & 7.3806657152 \\ 346 & \text { U1430 } & \text { A } & 10 & \text { H } & 1 & \text { W } & 71 & 84.75 & 84.75 & 7.4196605159 \\ 346 & \text { U1430 } & \text { A } & 10 & \text { H } & 2 & \text { W } & 71 & 86.25 & 86.25 & 7.4578907126 \\ 346 & \text { U1430 } & \text { A } & 10 & \text { H } & 3 & \text { W } & 69 & 87.73 & 87.73 & 7.4956111734 \\ 346 & \text { U1430 } & \text { A } & 10 & \text { H } & 4 & \text { W } & 69 & 89.23 & 89.23 & 7.5338413702 \\ 346 & \text { U1430 } & \text { A } & 10 & \text { H } & 5 & \text { W } & 71 & 90.75 & 90.75 & 7.5725813029 \\ 346 & \text { U1430 } & \text { A } & 10 & \text { H } & 6 & \text { W } & 71 & 92.25 & 92.25 & 7.6108114996 \\ 346 & \text { U1430 } & \text { A } & 10 & \text { H } & \text { CC } & \text { W } & 0 & 93.55 & 93.55 & 7.6439443368 \\ 346 & \text { U1430 } & \text { A } & 11 & \text { H } & 4 & \text { W } & 69 & 99.35 & 99.35 & 7.7917677643\end{array}$


Table 2 Samples used, biostratigraphic tie points, and related sedimentation rates at IODP Site U1430 (cm/ky) (Continued)

\begin{tabular}{|c|c|c|c|c|c|c|c|c|c|c|c|c|}
\hline Exp & Site & Hole & Core & Type & Sect & $\mathrm{A} / \mathrm{W}$ & $\begin{array}{l}\text { Offset } \\
(\mathrm{cm}) \\
\text { Mid }\end{array}$ & $\begin{array}{l}\text { CCSF-A (m) } \\
\text { (Tada et al. } \\
\text { 2015b) }\end{array}$ & $\begin{array}{l}\text { CCSF-D_- } \\
\text { Patched } \\
\text { (Irino et al. 2018) }\end{array}$ & Age (Ma) & $\begin{array}{l}\text { Sedimentation } \\
\text { rates }(\mathrm{cm} / \mathrm{ky})\end{array}$ & Comments \\
\hline 346 & U1430 & A & 11 & $\mathrm{H}$ & CC & W & 0 & 103.71 & 103.71 & 7.9028902029 & & \\
\hline 346 & U1430 & A & 12 & $\mathrm{H}$ & 4 & W & 69 & 109.56 & 109.56 & 8.0519879702 & & \\
\hline 346 & U1430 & A & 12 & $\mathrm{H}$ & CC & W & 0 & 114.02 & 114.02 & 8.1656590886 & & \\
\hline 346 & U1430 & A & 13 & $\mathrm{H}$ & 4 & W & 69 & 119.82 & 119.82 & 8.3134825161 & & \\
\hline 346 & U1430 & A & 13 & $\mathrm{H}$ & CC & W & 0 & 124.17 & 124.17 & 8.4243500867 & & \\
\hline 346 & U1430 & $A$ & 14 & $\mathrm{H}$ & 4 & W & 69 & 129.73 & 129.73 & 8.5660566826 & & \\
\hline 346 & U1430 & $A$ & 14 & $\mathrm{H}$ & CC & W & 0 & 134.19 & 134.19 & 8.679727801 & & \\
\hline 346 & U1430 & A & 15 & $\mathrm{H}$ & 4 & W & 69 & 139.65 & 139.65 & 8.8188857172 & & \\
\hline 346 & U1430 & A & 15 & $\mathrm{H}$ & $\mathrm{CC}$ & W & 0 & 144.11 & 144.11 & 8.9325568356 & & \\
\hline 346 & U1430 & A & 16 & $\mathrm{H}$ & 1 & W & 71 & 145.44 & 145.44 & 8.9664542767 & & \\
\hline 346 & U1430 & $A$ & 16 & $\mathrm{H}$ & 2 & W & 71 & 146.94 & 146.94 & 9.0046844734 & & \\
\hline 346 & U1430 & A & 16 & $\mathrm{H}$ & 3 & W & 71 & 148.44 & 148.44 & 9.0429146702 & & \\
\hline 346 & U1430 & A & 16 & $\mathrm{H}$ & 4 & W & 71 & 149.94 & 149.94 & 9.081144867 & & \\
\hline \multicolumn{8}{|c|}{ Tie point of Kamikuri et al. (2017) } & 150.68 & 150.68 & 9.100 & 3.924 & $\begin{array}{l}\text { We used the Last Occurrence } \\
\text { of L. magnacornuta } \\
\text { (Radiolaria), which occurred } \\
\text { at the mid depth of } 150.68 \mathrm{~m} \\
\text { CCSF-A. This bioevent has no } \\
\text { error margin. }\end{array}$ \\
\hline
\end{tabular}

$\begin{array}{lllllllllll}346 & \text { U1430 } & \text { A } & 16 & \text { H } & 5 & \text { W } & 69 & 151.42 & 151.42 & 9.117 \\ 346 & \text { U1430 } & \text { A } & 16 & \text { H } & 6 & \text { W } & 71 & 152.94 & 152.94 & 9.151 \\ 346 & \text { U1430 } & \text { A } & 16 & \text { H } & \text { CC } & \text { W } & 0 & 154.41 & 154.41 & 9.184 \\ 346 & \text { U1430 } & \text { A } & 17 & \text { H } & 1 & \text { W } & 71 & 156.81 & 156.81 & 9.239 \\ 346 & \text { U1430 } & \text { A } & 17 & \text { H } & 2 & \text { W } & 71 & 158.31 & 158.31 & 9.273 \\ 346 & \text { U1430 } & \text { A } & 17 & \text { H } & 3 & \text { W } & 69 & 159.79 & 159.79 & 9.306 \\ 346 & \text { U1430 } & \text { A } & 17 & \text { H } & 4 & \text { W } & 71 & 161.33 & 161.33 & 9.341 \\ 346 & \text { U1430 } & \text { A } & 17 & \text { H } & 5 & \text { W } & 69 & 162.83 & 162.83 & 9.375 \\ 346 & \text { U1430 } & \text { A } & 17 & \text { H } & 6 & \text { W } & 71 & 164.33 & 164.33 & 9.409 \\ 346 & \text { U1430 } & \text { A } & 17 & \text { H } & \text { CC } & \text { W } & 0 & 165.78 & 165.78 & 9.442 \\ \text { Tie point of Kamikuri et al. (2017) } & & & & 188.24 & 188.24 & 9.950\end{array}$

4.418 We used the Last Occurrence of L. magnacornuta (Radiolaria), which occurred at the mid depth of 188.24 m CCSF-A. This bioevent has an error margin of $0.25 \mathrm{Ma}$ from 1.20 to $0 \mathrm{Ma}$, while Phorticium spp. have relative abundances twice as high as the mean value between 7.27 and 3.28 Ma (Fig. 4 and Additional file 1).

The L. minor group and S. tenuispina group are extant species, although their ecology is not well understood. The L. minor group, which has an unsettled taxonomy and is often named as Lithelius minor Jørgensen (e.g., Matsuzaki et al. 2015a), likely inhabits intermediate water in the subtropical North Pacific (e.g., Matsuzaki et al. 2016). This species is also related to subtropical-transitional areas of the North Pacific (Motoyama and Nishimura 2005). The vertical distribution of $S$. tenuispina group is not well understood, but this species is probably related to transitional to subarctic areas in the Northwest Pacific, according to Kamikuri et al. (2008) (named as Stylodictya stellata Bailey in Kamikuri et al. 2008). Both species occurred sporadically since 9.5 $\mathrm{Ma}$ and their mean relative abundances are $2 \%$ 
$(\mathrm{SD}=3.6 ; N=154)$ and $1.8 \%(\mathrm{SD}=2.8 ; N=154)$ for the $S$. tenuispina and $L$. minor groups, respectively (Fig. 4 and Additional file 1).

The $S$. venustum/bensoni groups are extinct, but they probably inhabited the middle to high latitudes of the North Pacific during the Neogene (Kamikuri 2010). The L. polyacantha group dominated the radiolarian assemblage between about 15 and $7 \mathrm{Ma}$ at ODP Site 884 (south of the Bering Sea), Site 887 (southern Gulf of Alaska), and Site 1151 (off Northeast Japan) (Kamikuri et al. 2004, 2007), and therefore, this species is also probably related to the middle to high latitudes of the North Pacific during the Neogene. S. venustum/bensoni groups occurred throughout the last $9.5 \mathrm{Ma}$ and their mean relative abundance is $1.5 \%(\mathrm{SD}=3.1 ; \mathrm{N}=$ 154), with highest relative abundance between 5.3 and 3.06 Ma (Fig. 3 and Additional file 1). The L. polyacantha group occurred between 9.57 and $2.3 \mathrm{Ma}$, and its mean value is $1.6 \%(\mathrm{SD}=2.1 ; N=127)$ (Fig. 3 and Additional file 1).

S. delmontensis/peregrina are extinct species that were widely distributed in the North Pacific during the Mio-Pliocene (Motoyama 1996; Kamikuri 2012; Kamikuri et al. 2007; Oseki and Suzuki 2009; Romine 1985). S. delmontensis/peregrina only occur sporadically between 9.57 and $3.89 \mathrm{Ma}$ and their mean relative abundance is $1.5 \%(\mathrm{SD}=4.4 ; N=106)$ (Fig. 4 and Additional file 1$)$. Relative abundance higher than the mean value only occurs between 9.57 and 7.21 Ma (Fig. 4 and Additional file 1). These species are abundant in sediment core samples from the North Pacific (e.g., Romine 1985), while they have only sporadic occurrence in the Japan Sea. The ecology of these species is also under debate. Romine (1985) showed that despite a wide distribution in the North Pacific, these species were probably related to temperate-subarctic areas. Casey (1982) suggested that these species may have inhabited shallow water at high latitudes and intermediate water at low latitudes, while Kamikuri (2012) reported that each species had its own high-latitude and low-latitude forms, which make the diagnostic use of $S$. delmontensis/peregrina problematic. However, Motoyama (1996) suggested that S. delmontensis/peregrina are probably a marker of warm water in the Northwest Pacific. In addition, previous studies in the Japan Sea showed that the relative abundances of $S$. delmontensis/peregrina covaried together with those of Collosphaera spp. (Kamikuri and Motoyama 2007). Collosphaera spp. belong to the order Collodaria, which includes species inhabiting shallow water of subtropical to temperate areas (Suzuki and Not 2015; Biard et al. 2016, 2017). Therefore, in this study, we assumed that the presence of $S$. delmontensis/peregrina suggests subtropical-temperate water of the North Pacific.

\section{Relative abundance of minor species}

We have defined species or species groups as minor when their relative abundance is between 0 and $10 \%$ of the total assemblage. These minor species are Spongopyle osculosa Dreyer; Larcopyle weddellium Lazarus, Faust, and Popova-Goll group; and Cornutella profunda Ehrenberg and Carpocanistrum papillosum (Ehrenberg) group (Figs. 3 and 4).

The species $S$. osculosa is a transitional-subarctic to subarctic species inhabiting intermediate water depths in the North Pacific (Kling and Boltovskoy 1995; Kamikuri et al. 2008; Matsuzaki and Itaki 2017). This species has a sporadic occurrence at Site U1425 and its mean relative abundance is $1 \%(\mathrm{SD}=1.4 ; \mathrm{N}=154)$. This species' relative abundance is higher than the mean value between 4 and $3 \mathrm{Ma}$, with a maximum of $10.5 \%$ at $3.56 \mathrm{Ma}$ (Fig. 3 and Additional file 1).

The species L. weddellium is known as a subarctic-arctic species in the North Pacific (Tanaka and Takahashi 2008; Kamikuri et al. 2008; Matsuzaki and Itaki 2017). At the middle to low latitudes of the North Pacific, this species is less abundant and inhabits intermediate water depths only (Matsuzaki et al. 2016). This species has also been abundant at high latitudes of the Southern Hemisphere since 10 Ma (i.e., ODP Sites 689-690 in the Weddell Sea) (Lazarus et al. 2005). Therefore, this species is likely related to subpolar-polar areas of both hemispheres (bi-polar species). This species' mean relative abundance is $1.6 \%(\mathrm{SD}=1.8 ; \mathrm{N}=154)$, although its relative abundance never exceeds $1.0 \%$ after $1.47 \mathrm{Ma}$ (Fig. 4 and Additional file 1). This species' relative abundance is generally higher than the mean value between 7.91 and 5.05 Ma (Fig. 4 and Additional file 1).

C. profunda is an extant species that inhabits water deeper than 300 m (Renz 1976; Casey et al. 1979; Takahashi 1991; Takahashi and Honjo 1981), while C. papillosum, which is also an extant species, inhabits water deeper than about $1000 \mathrm{~m}$ in the North Pacific (Takahashi 1991; Takahashi and Honjo 1981). At Site U1425, C. profunda occurs between 9.57 and $2.56 \mathrm{Ma}$, and its mean relative abundance is $1.8 \%(\mathrm{SD}=1.7 ; N=126)$. The C. papillosum group occurs between 9.57 and $3.89 \mathrm{Ma}$, and its mean relative abundance is $0.9 \%$ (SD = 1.5; $\mathrm{N}=106$ ) (Fig. 4 and Additional file 1). C. papillosum has a higher relative abundance exceeding $2 \%$ between 9.57 and $7.91 \mathrm{Ma}$ (Fig. 4 and Additional file 1). Additionally, the species $C$. profunda shows relative abundance of $1-8 \%$ from 9.57 to $2.79 \mathrm{Ma}$ (Fig. 4 and Additional file 1).

\section{Site U1430}

According to Tada et al. (2015b) and Kamikuri et al. (2017), a hiatus is present at Site U1430 in the upper part of unit IIB (about $83 \mathrm{~m}$ CCFS-D according to Tada 
et al. 2015b) and the stratigraphic interval between the D. bullatus (4.3-3.8 Ma) and L. parallelipes zones (6.8/ 7.0 Ma to 7.2/7.4 Ma) is missing (e.g., Kamikuri et al. 2017). Therefore, we only describe the abundance of species that can support the data obtained at Site U1425 for the reconstruction of the paleoceanography of the Pleistocene and late Miocene (Fig. 5). Species related to arctic and subarctic areas, such as A. setosa, C. borealis, and $C$. davisiana, occur since $3 \mathrm{Ma}$ (Fig. 5). The mean relative abundance of $A$. setosa is $4.04 \%(\mathrm{SD}=5.03 ; N=$ 11), while the means of $C$. borealis and $C$. davisiana are 7.98\% (SD = 9.1; $N=14)$ and 9.27\% ( $\mathrm{SD}=23.41 ; \mathrm{N}=14)$, respectively (Fig. 5 and Additional file 2). Relative abundances of $C$. sphaeris and the $S$. lineata/arachnea group could not be estimated between 7.4 and ca. 4 Ma because of the hiatus (Fig. 5). Abundances of Phorticium spp. and Tetrapyle spp., which are warm-water index species (see above), are also affected by this hiatus. The mean relative abundances of Phorticium spp. and Tetrapyle spp. are respectively $1.13 \%(\mathrm{SD}=2.9 ; N=51)$ and $0.68 \%(\mathrm{SD}=1.48 ; N=51)$ (Fig. 5 and Additional file 2). Tetrapyle spp. has a relative abundance higher than the mean value for the periods between 9.24 and $9.15 \mathrm{Ma}$, 8.93 and $8.68 \mathrm{Ma}, 7.64$ and $7.54 \mathrm{Ma}$, and 1.15 and $0 \mathrm{Ma}$ (Fig. 5 and Additional file 2). The S. delmontensis/peregrina group occurs only sporadically between 8.41 and 8.31 Ma and 7.38 and 7.30 Ma, with an abundance peak exceeding 30\% of the total assemblage (Fig. 5 and Additional file 2). C. nakasekoi occurs continuously between 9.44 and $7.38 \mathrm{Ma}$, and its mean relative abundance is $11.58 \%(\mathrm{SD}=8.60 ; N=32)$ (Fig. 5 and Additional file 2). C. papillosum occurs between 9.44 and $7.38 \mathrm{Ma}$, and its mean relative abundance is $1.57 \%(\mathrm{SD}=2.29 ; N=33)$, while C. profunda occurs between 9.44 and $2.52 \mathrm{Ma}$ with a mean relative abundance of $0.84 \%(\mathrm{SD}=1.50$; $N=40$ ) (Fig. 5 and Additional file 2).

\section{Discussion}

During the Mio-Pliocene, the local paleoceanography of the Japan Sea was closely related to regional tectonic activity and the progressive uplift of the Japanese islands. During the Miocene until 10.5 Ma, the Japan Sea was connected to the North Pacific Ocean via seaways located around the edges of the East China and Yellow seas, Fossa Magna basin, the subsided Tohoku area at mid-latitudes, between northern Honshu and southern Hokkaido islands, and around Sakhalin Island (e.g., Fukusawa 1988; Fujii et al. 1992; Iijima and Tada 1990; Sato 1994; Kano et al. 1991; Kharakhinov 2010; Kobayashi and Tateishi 1992; Kosaka et al. 1992; Sagayama 2002; Yahata 2002; Yoshida et al. 2013). Between ca. 10.5 and $7 \mathrm{Ma}$, the Japan Sea was connected to the North Pacific via relatively shallow seaways around the Fossa Magna, subsided Tohoku area, and Sakhalin Island, where neritic sedimentary facies $(<200 \mathrm{~m})$ are recognized (e.g., Iijima and Tada 1990; Sato 1994; Yoshida et al. 2013; Kharakhinov 2010; Fukusawa 1988). However, a relatively deep seaway was reported between the northern Honshu and southern Hokkaido islands (e.g., Fukusawa 1988; Iijima and Tada 1990). Tada (1994) suggested that the sill depth of this seaway would have been between 500 and $1000 \mathrm{~m}$ based on the sediments and fossil facies reported in Iijima and Tada (1990). Around 8 and $7 \mathrm{Ma}$, the northeast Japan Islands uplifted because of tectonic activity, which probably closed the Tohoku and Fossa Magna seaways, and thus the Japan Sea was only connected to the North Pacific via seaways located around the southern Hokkaido and Sakhalin islands until 3.1 Ma (e.g., Iijima and Tada 1990; Kano et al. 1991; Sato 1994; Yoshida et al. 2013; Kharakhinov 2010; Fukusawa 1988). At $3.1 \mathrm{Ma}$, most studies agree that the seaway around the East China and Yellow seas re-opened (Itaki 2016; Kitamura and Kimoto 2006; Kitamura et al. 2001; Tada 1994; Wang et al. 2014). In the discussion below, we propose to reconstruct the paleoceanography of shallow to deep waters with each connecting seaway configuration taken into account.

In the following discussion, we use the following nomenclature: southern seaway is the seaway located around the edge of the East China and Yellow seas, central seaway is the Fossa Magna basin, eastern seaway is the subsided Tohoku area at mid-latitudes, northern seaway is the area between northern Honshu and southern Hokkaido islands, and northernmost seaway is the area around Sakhalin Island.

\section{Evolution of the sill depth of the northern seaway since 9.5 Ma}

According to Iijima and Tada (1990), whose paleobathymetrical estimation relied upon sedimentological, micropaleontological (benthic foraminifera), and macrofossil (e.g., bivalves) analyses, the sill depths of the Japan Sea northern seaway are estimated as middle to upper bathyal water depths (500-1000 m) during the late Miocene (ca. 10-6 Ma). Our new dataset provides additional constraints on the estimation of the northern strait sill depths. At Sites U1425 and U1430, the time interval between ca. 9.5 and $7.8 \mathrm{Ma}$ is characterized by occurrences of C. profunda inhabiting water below $300 \mathrm{~m}$ and $C$. papillosum inhabiting water between 1000 and $3000 \mathrm{~m}$ deep in the North Pacific (Figs. 4, 5 and 6) (Takahashi and Honjo 1981; Takahashi 1991). These species are absent in the modern Japan Sea (e.g., Itaki 2003; Itaki 2016; Motoyama et al. 2016a). Itaki (2016) suggested that the absence of these species in the modern Japan Sea is due to the geographical isolation of the intermediate and deep water of the Japan Sea from the North Pacific and thus suggested that these species are related to 


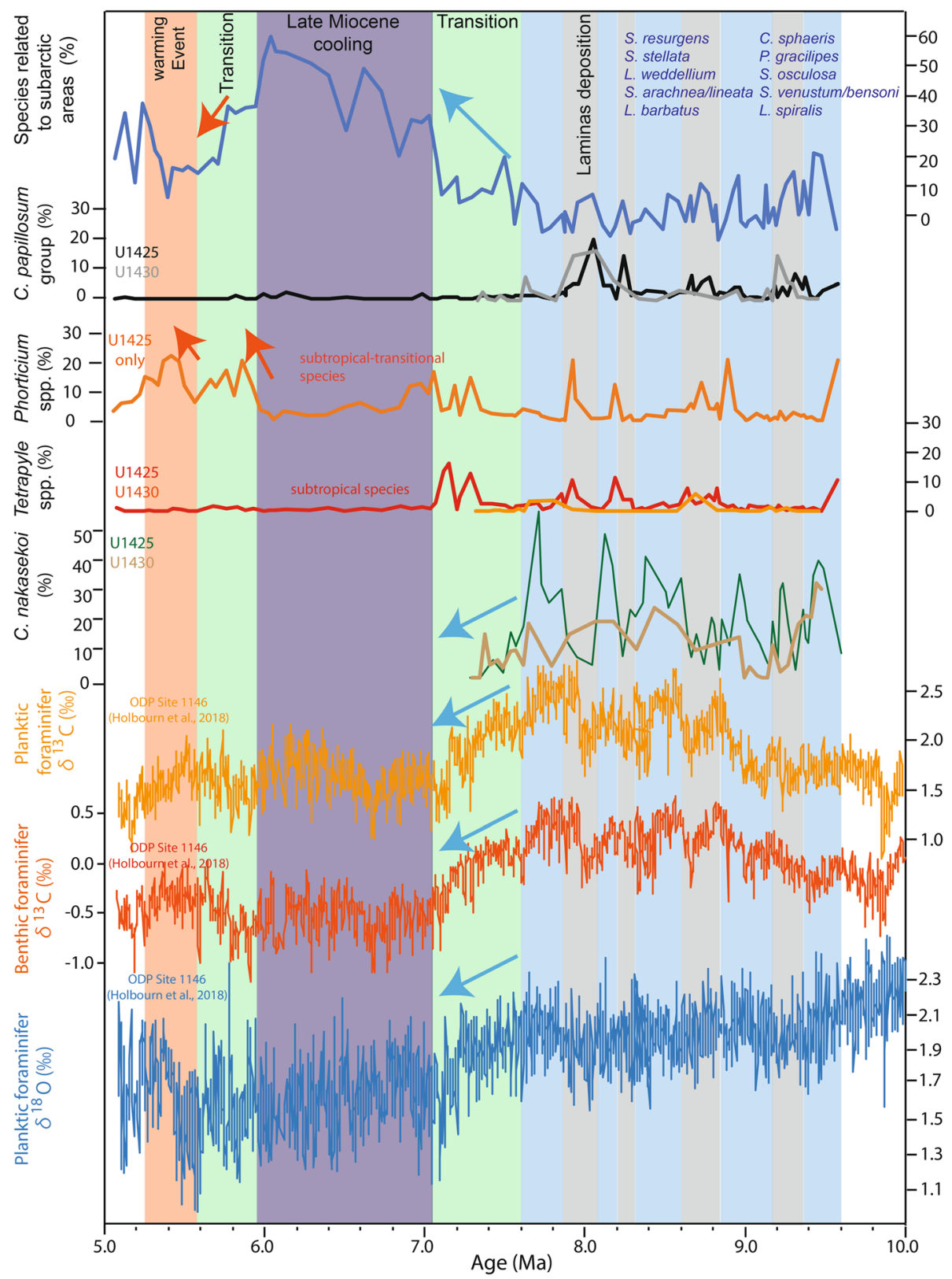

Fig. 6 Details of the late Miocene paleoceanography of the Japan Sea (10-5 Ma). We compare relative abundances of C. nakasekoi, Tetrapyle spp. and C. papillosum from Sites U1425 and U1430 between 9.5 and $7 \mathrm{Ma}$. The interval between 7.0 and $5.0 \mathrm{Ma}$ shows relative abundance of Phorticium spp. and species related to subarctic water (the selected species are listed at the upper right in the figure). These data are compared to oxygen and carbon isotope data for planktic and benthic foraminifer obtained from the South China Sea (ODP 1146, Holbourn et al. 2018)

the deep water of the North Pacific. Therefore, in this study, we assumed that $C$. profunda and C. papillosum are indicators of deep water of the North Pacific. Thus, our data imply that between 9.5 and $7.8 \mathrm{Ma}$ intermediate to deep water of the North Pacific probably flowed into the Japan Sea through the northern seaway, which had water depths between 500 and $1000 \mathrm{~m}$, because other seaways open at that time (central, east, and northernmost) were too shallow $(<200 \mathrm{~m})$ to enable deep-water exchanges. Although the passive upwelling of deep-water current may have also had an influence on these inflows of deep water, because our radiolarian data are in agreement with other sedimentological data estimating the sill depth of the northern seaway (e.g. Iijima and Tada 1990), we can consider that the effect of the passive upwelling is minor. Therefore, our new data, combined with the previous data of Iijima and Tada (1990), suggest that the sill depth of the northern seaway was likely close to $1000 \mathrm{~m}$ between 9.5 and $7.8 \mathrm{Ma}$. The very low abundance or absence of C. papillosum and the progressive 
decrease in C. profunda relative abundance since $7.8 \mathrm{Ma}$ implies a probable shoaling of the northern seaway between 7.8 and $3 \mathrm{Ma}$, with the northern seaway probably having a sill depth shallower than $300 \mathrm{~m}$ at ca. $3 \mathrm{Ma}$. This is likely related to regional tectonic activity and progressive uplift of the Japanese islands since 8-7 Ma, when the stress regime in the Japanese islands and eastern Japan Sea changed from extensional to compressional (e.g., Jolivet et al. 1994; Sato 1994; Yoshida et al. 2013).

\section{Paleoceanography of the Japan Sea between 9.5 and 7.0 Ma}

During this interval, the Japan Sea was connected to the North Pacific via shallow seaways at the central, east, and northernmost Japan Sea and via a deep seaway in the north. Our data show intermittent high abundances of Tetrapyle spp. and S. delmontensis/peregrina at Sites U1425 and U1430 and, thus, relatively warm shallow water probably prevailed intermittently (Fig. 6). Because S. delmontensis/peregrina had a relatively wide geographical distribution and their ecology remains controvertible, we use mainly Tetrapyle spp. to discuss the local paleoceanography.

Tetrapyle spp. occur episodically at ODP Site 302 in the central Japan Sea (Kamikuri and Motoyama 2007) and thus support our findings that the Japan Sea was influenced by warm water of the North Pacific between 9.5 and 7.0 Ma. Taking into account that only the northernmost, northern, eastern, and central seaways were open between 10 and 7 Ma (e.g. Iijima and Tada 1990; Kano et al. 1991; Kharakhinov 2010; Sagayama 2002; Sato 1994), warm current must have flowed into the Japan Sea through one of these seaways. Previous studies did not report Tetrapyle spp. flowing the northern Japan outcrops (Motoyama 1992; Motoyama 1993), middle-high latitudes of the Northwest Pacific (ODP Sites 1151/1152) (Kamikuri et al. 2004), the Bering south of Sea/Gulf of Alaska (ODP Sites 884-887) (Kamikuri et al. 2007), or Hokkaido area outcrops (e.g., Shinzawa et al. 2009; Motoyama and Nakamura 2002; Motoyama et al. 2016b). As extant Tetrapyle spp. live in warm water and always bear algal symbionts at the subfamily level (e.g., Zhang et al. 2018), shallow-warm waters were not flowing in these regions. Thus, warm water of the North Pacific probably did not flow into the Japan Sea via the northern and northernmost seaways, and the eastern and central Japan Sea seaways are the most plausible pathways for enabling the inflow of warm water into the Japan Sea from the North Pacific. Interestingly, the abrupt decrease in Tetrapyle spp. abundances at ca. $7 \mathrm{Ma}$ also corresponds to the timing of emergence of lands in the Tohoku area and the closure of the central seaway (e.g., Fujii et al. 1992; Sato 1994; Yoshida et al. 2013). In consideration of the intermittent occurrence of Tetrapyle spp. between 9.5 and
$7 \mathrm{Ma}$, we point out that the northern limit of Tetrapyle spp. was probably around the Tohoku area (eastern seaway) during the late Miocene, and thus warm water probably flowed intermittently via the central and eastern seaways between 9.5 and 7 Ma (Fig. 7).

The time interval between 9.5 and $7.0 \mathrm{Ma}$ is also characterized by high abundance of C. nakasekoi (about 30-60\%) and intermittent abundance of C. papillosum fluctuating between 1 and 10\% (Fig. 6). The intermittent fluctuation pattern for $C$. papillosum is roughly similar to those of Tetrapyle spp. (Fig. 6). We know that $C$. papillosum inhabits water depths between 1000 and $3000 \mathrm{~m}$ in the North Pacific and its presence implies that the sill depth of the northern seaway was probably about $1000 \mathrm{~m}$. Previous studies based on measurement of $\delta^{13} \mathrm{C}$ in benthic foraminifera imply that a layer with low dissolved oxygen content existed in the North Pacific between 1000 and $3000 \mathrm{~m}$, the so-called OMZ (Woodruff and Savin 1989). If we assume that the habitat zone of C. papillosum was similar throughout the Neogene, C. papillosum may have inhabited the OMZ water during the late Miocene. This hypothesis is strengthened by the fact that laminations of diatom ooze occurring within a dark organic-rich layer extending to a thickness of about $1.5 \mathrm{~m}$ were recognized at about $287 \mathrm{~m}$ CCSF-D, which corresponds to an age of ca. $8 \mathrm{Ma}$ (Irino et al. 2018; Kamikuri et al. 2017). Interestingly, the highest relative abundance (about 10\%) of $C$. papillosum is recorded at ca. $8 \mathrm{Ma}$ at Sites U1425 and U1430 (Fig. 6), when the thickest lamina were deposited (Tada et al. 2015a). Other laminations are also recorded at depths below $287 \mathrm{~m}$ CCSF-D; however, these were not as thick, so their positions were not reported in Tada et al. (2015a). Interestingly, the relative abundance of $C$. papillosum was also intermittent before $8 \mathrm{Ma}$, but its relative abundance was much lower (Fig. 6). Therefore, it is possible that the occurrence of C. papillosum is related to the deposition of laminas. Based on Watanabe et al. (2007), laminas are deposited in the Japan Sea when the bottom water is poor in oxygen. Therefore, it is probable that the $C$. papillosum abundance implies oxygen-poor bottom water in the Japan Sea. Thus, when C. papillosum abundance is high, it is probable that OMZ water from the North Pacific was flowing into the Japan Sea, while when the C. papillosum relative abundance was lower or close to $0 \%, \mathrm{OMZ}$ water probably was not flowing. To explain this intermittent fluctuation in C. papillosum, one plausible explanation is the glacioeustatic sea-level variation, which was already proposed by Tada (1994), who assumed that orbitally paced glacioeustatic sea-level changes probably caused shoaling and deepening of the northern seaway (ca. $1000 \mathrm{~m}$ ), although a shoaling/deepening of the OMZ itself cannot be neglected (Fig. 7). However, as said above, the 


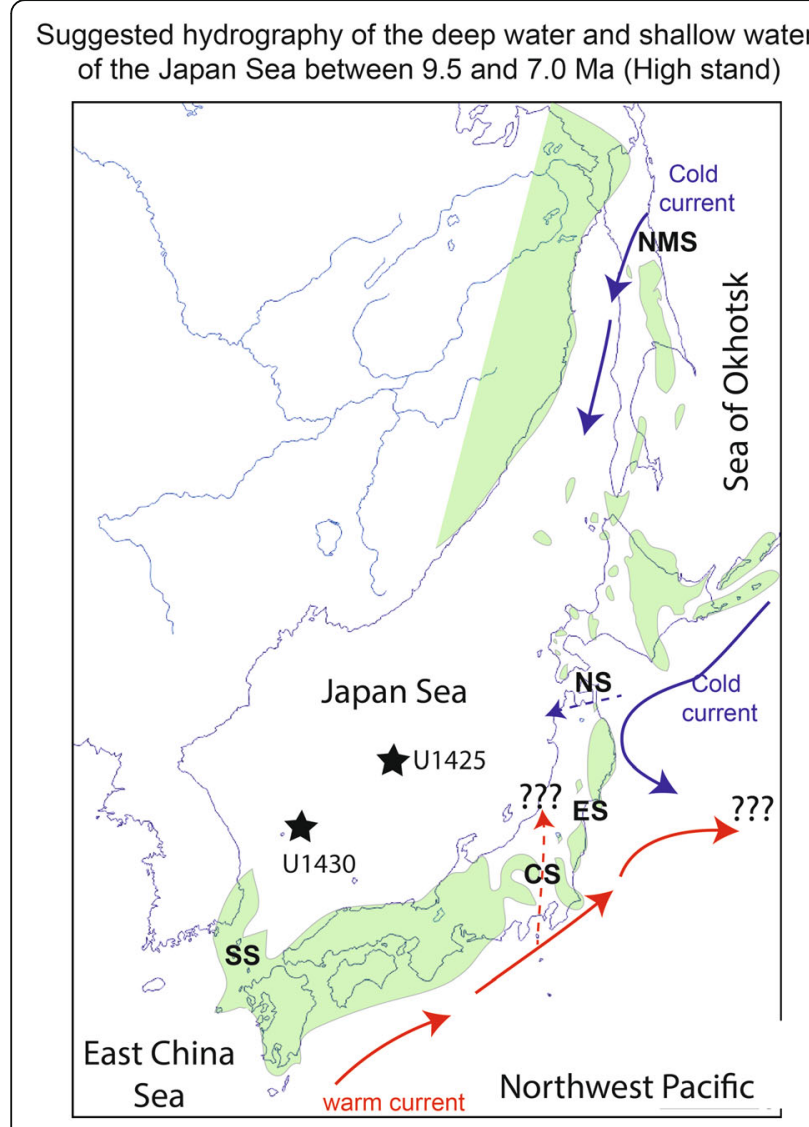

Suggested hydrography of the deep water and shallow water of the Japan Sea between 9.5 and $7.0 \mathrm{Ma}$ (Low stand)
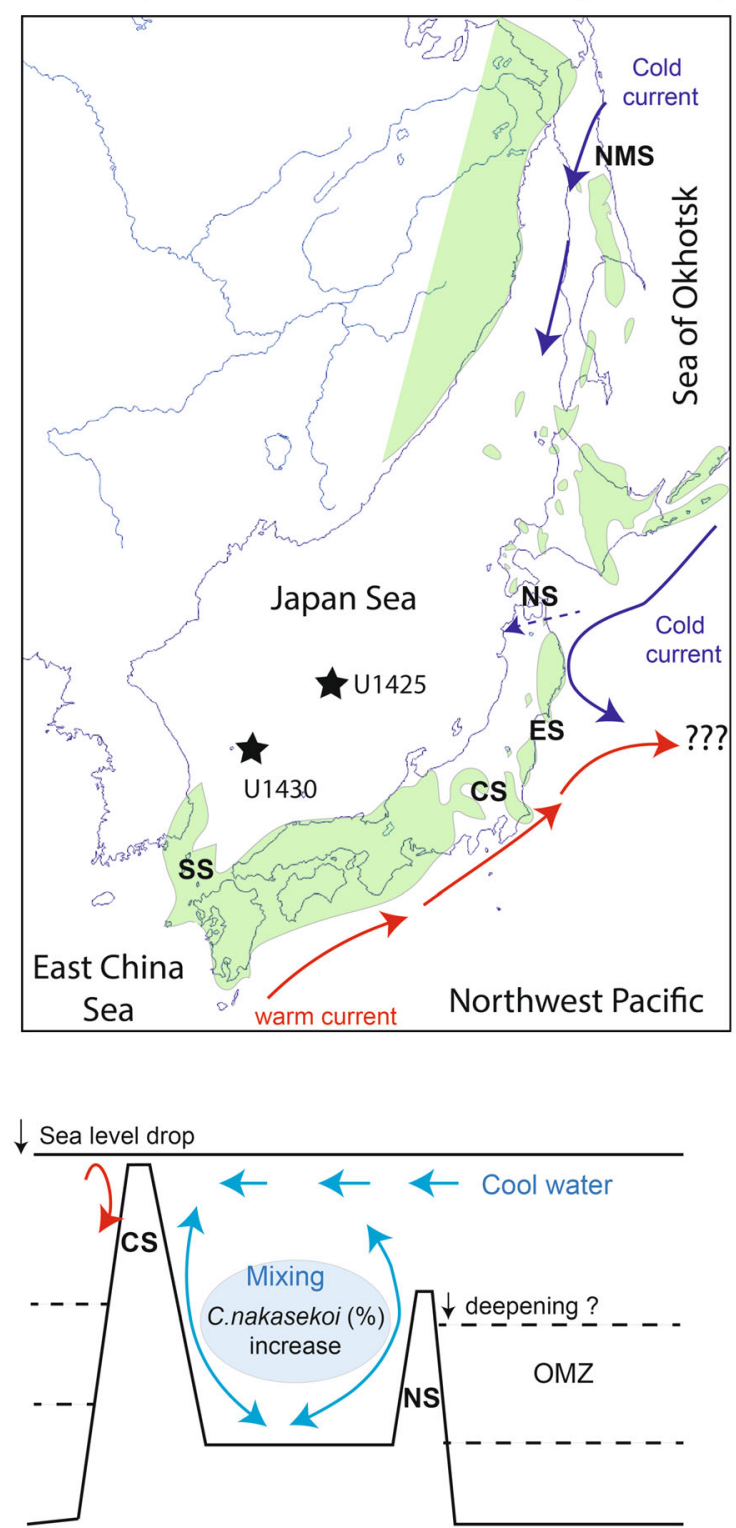

SS: Southern Seaway; CS: Central Seaway; ES: Eastern Seaway; NS: Northern Seaway; NMS: Northernmost Seaway

Fig. 7 Suggested hydrography of the deep water and shallow water of the Japan Sea between 9.5 and $7.0 \mathrm{Ma}$

intermittent fluctuation of Tetrapyle spp. is roughly synchronous to that of C. papillosum. We have already shown that warm water of the North Pacific probably flowed into the Japan Sea via the eastern and northeastern seaways, which had relatively shallow water depths. Therefore, it is probable that changes in the sill depths of the connecting seaways (northern, eastern, and central) in relation to the glacioeustatic sea-level changes influenced OMZ and warm water inflow from the North Pacific into the Japan Sea at that time.

In contrast, the relative abundance $C$. nakasekoi increased when $C$. papillosum and Tetrapyle spp. had a low relative abundance. The concern is that $C$. nakasekoi is a species reported in the Japan Sea only between ca. 10 and 7 Ma (Kamikuri and Motoyama 2007; Kamikuri et al. 2004; Motoyama 1996), and its ecology remains 
unknown. Previous studies reported that C. nakasekoi is absent in the North Pacific Ocean and only constituted about $1 \%$ of the total assemblage in samples from outcrops in Hokkaido Island, while this species exceeded $20 \%$ of the total assemblage in the Japan Sea (e.g., Kamikuri et al. 2004; Motoyama et al. 2016b; Shinzawa et al. 2009; this study). As we assumed that the sill depth of the northern seaway was ca. $1000 \mathrm{~m}$ between 9.5 and 7.8 Ma, one plausible explanation is that C. nakasekoi lived in waters deeper than $1000 \mathrm{~m}$, and thus C. nakase$k o i$ would have been trapped in the Japan Sea. However, it seems that high abundance of $C$. nakasekoi occurred when the relative abundances of Tetrapyle spp. and/or Phorticium spp. were low (Fig. 6). This implies that the C. nakasekoi relative abundance increased when the relative abundances of species related to warm water decreased. Therefore, although the specific distribution of C. nakasekoi suggests that this could be an intermediate-to-deep-water species restricted to the Japan Sea, we cannot assume that this species is not related to a cold marine environment. In fact, the more probable explanation is that $C$. nakasekoi is related to poorly stratified water conditions. Indeed, Tada (1994) suggested that the $\mathrm{OMZ}$ was nutrient-rich and probably had a high salinity based on the interpretation of the value of $\delta^{13} \mathrm{C}$ reported by Woodruff and Savin (1989). However, warm water is known for having a low density (e.g., Ruddiman 2001). Therefore, when the OMZ and warm water flowed from the North Pacific into the Japan Sea, the water column was likely well stratified (Tada 1994). In such a context, we observed low relative abundance of $C$. nakasekoi (Figs. 6 and 7). In contrast, when there was no inflow of $\mathrm{OMZ}$ and warm water from the North Pacific, it is likely that the water masses in the Japan Sea were less stratified, and Tada (1994) suggested that the paleoceanographic condition would have been more or less similar to the present Japan Sea, where deep water is well ventilated because of a vertical mixing of water masses (Fig. 7). Therefore, it is probable that C. nakasekoi developed in the Japan Sea when the water column was probably relatively well mixed, and thus it seems that $C$. nakasekoi prefers water masses that are well oxygenated (Figs. 6 and 7).

\section{Paleoceanography of the Japan Sea during the late Miocene and early Pliocene with only the northern strait open (ca. 7-5.0 Ma)}

From 7 to $5 \mathrm{Ma}$, the northern and northernmost seaways were the only gateways enabling water exchanges between the Japan Sea and the North Pacific. As suggested above, the sill depth of the northern strait was likely shallower than $1000 \mathrm{~m}$ but deeper than $300 \mathrm{~m}$ between ca. 7.8 and $3 \mathrm{Ma}$ because of the presence of C. profunda $(>300 \mathrm{~m})$ and low relative abundance/or absence of C. papillosum (> $1000 \mathrm{~m}$ ) (Fig. 6). This suggests that only intermediate to shallow water could have been exchanged between the Japan Sea and North Pacific at that time. Radiolarian data at Site U1425 suggest enhanced cooling conditions in the Japan Sea between 7.0 and 5.8 Ma, which was characterized by an increase in abundances of the subarctic-transitional species, exceeding $30 \%$ of the total assemblage during this interval (Fig. 5). Our data therefore suggest that a cold marine environment prevailed in the Japan Sea at that time.

Recently, Herbert et al. (2016) used the alkenone unsaturation index to show that a synchronous cooling occurred in shallow water at high latitudes in the Northern Hemisphere between ca. 7.5 and 5.5 Ma. This cooling was interpreted as a transient glaciation of the Northern Hemisphere and is called the late Miocene cooling (Herbert et al. 2016). Other studies suggested that during this cooling, an increase in $\mathrm{C}_{4}$ plants was recorded, and thus the climate likely became drier (e.g., Cerling et al., 1997; Holbourn et al. 2018; Huang et al., 2007). Additionally, recent analysis of Asian eolian dust from the Japan Sea also suggested a temporal drying of Central Asia ca. 8$7 \mathrm{Ma}$ (Shen et al. 2017). Holbourn et al. (2018) used $\delta^{18} \mathrm{O}$ and $\delta^{13} \mathrm{C}$ to measure benthic and planktic foraminifera at ODP Site 1146 (South China Sea) and suggested a cooling at that time because of enhanced EAWM, which probably increased the biological pump and drastically modified the carbon cycle. In Fig. 6, we compare our radiolarian data to the isotope data of Holbourn et al. (2018). The cooling implied by radiolarian assemblages is synchronous with the shift recorded in isotope data from the South China Sea, and thus the cooling implied by radiolarian assemblages is likely related to the late Miocene cooling and the enhanced EAWM. Cooling at high latitudes is generally associated with a southward shift of the subarctic front (e.g., McClymont et al., 2008), while enhanced EAWM generally strengthened winds blowing from north to south because of a Siberian high and Aleutian low (e.g., Tada et al. 2016). Therefore, rather than a local increase in cold radiolarian species productivity because of a cooling climate, it is likely that the influence of cold water currents was stronger during the late Miocene cooling, and thus a larger amount of cold water was transported from high latitudes to the marginal sea. This implies that this cold current influenced the Japan Sea via the northern and northernmost seaways located between northern Honshu and southern Hokkaido islands and around Sakhalin Island because other seaways were closed at that time (Fig. 8).

In this study, at Site U1425, abundances of subarctic-transitional species drastically decreased between 5.8 and 5.2 Ma, while abundances of species related to shallow water of transitional-subtropical (Phorticium spp.) 
areas increased (by about 20\%) (Fig. 6). Radiolarian data imply that cold water continued to flow into the Japan Sea from the northernmost seaway (Sakhalin area) because the relative abundance of transitional-subarctic species was still fluctuating between 10 and 20\% (Fig. 6). However, relatively warm water probably flowed at the same time from the northern seaway (northern Honshu and Southern Hokkaido islands) because the relative abundance of subtropical-transitional species was about $20 \%$, while their abundance was less than $5 \%$ between 7 and 5.8 Ma (Fig. 8). Previously published data for mollusca fossils confirmed our hypothesis by showing that temperate conditions prevailed in the Japan Sea during that time (Ogasawara 1994). Around 5 Ma (early Pliocene), Earth experienced a warm climate with relatively high atmospheric $\mathrm{pCO}_{2}$ (e.g., Fedorov et al. 2013). At the same time, the tropical warm pool likely expanded (e.g., Brierley et al. 2009). Therefore, considering that we have used an age model relying on biostratigraphy of radiolarians and an error margin of ca. $0.2 \mathrm{Myr}$ (Kamikuri et al. 2017), the higher abundances of transitional-subtropical species between ca. 5.8 and $5.2 \mathrm{Ma}$ are probably related to the warm conditions prevailing throughout the Earth, which plausibly caused an expansion of the tropical warm pool and a northward shift of the subtropical front, enabling inflow of relatively temperate-warm water into the Japan Sea through the northern strait.
Drastic cooling of the subsurface and intermediate waters during the mid-Pliocene (ca. 5.0-3.8 Ma)

Our radiolarian data from the Japan Sea show that the $S$. arachnea/lineata group, an intermediate-water species restricted to the polar to subpolar areas of the Northern Hemisphere (e.g., Boltovskoy and Correa 2016), dominates our assemblages between ca. 5.0 and 3.8 Ma (Fig. 9). The S. arachnea/lineata group reported in previous paleoceanographic and biostratigraphic studies showed a low abundance $(<5 \%)$ because this group is relatively small (about $50 \mu \mathrm{m}$ ), and most of the previous studies processed the sediments with $63-\mu \mathrm{m}$ mesh sieves (e.g., Motoyama 1996; Kamikuri et al. 2004, 2007, 2009). Despite this technical issue, increases and decreases in the abundance of this group have been reported off the northeastern Japanese coast and in the subarctic North Pacific south of the Bering Sea (ODP Sites 1151 and 884 ) at ca. 4.7 and $4.0 \mathrm{Ma}$ (appendix of Kamikuri et al. 2004, 2007). In the southern Gulf of Alaska (ODP Site 887 ) and in the Equatorial Pacific (ODP Sites 845 and 1241), this species was absent (Kamikuri et al. 2007; Kamikuri et al. 2009). These findings, combined with our data, suggest that a drastic cooling occurred in the subsurface to intermediate waters of the Japan Sea and the Northwest and Central North Pacific almost simultaneously during the mid-Pliocene. This may have been caused by a southward flow of subarctic subsurface to intermediate water from high latitudes of the Northwest
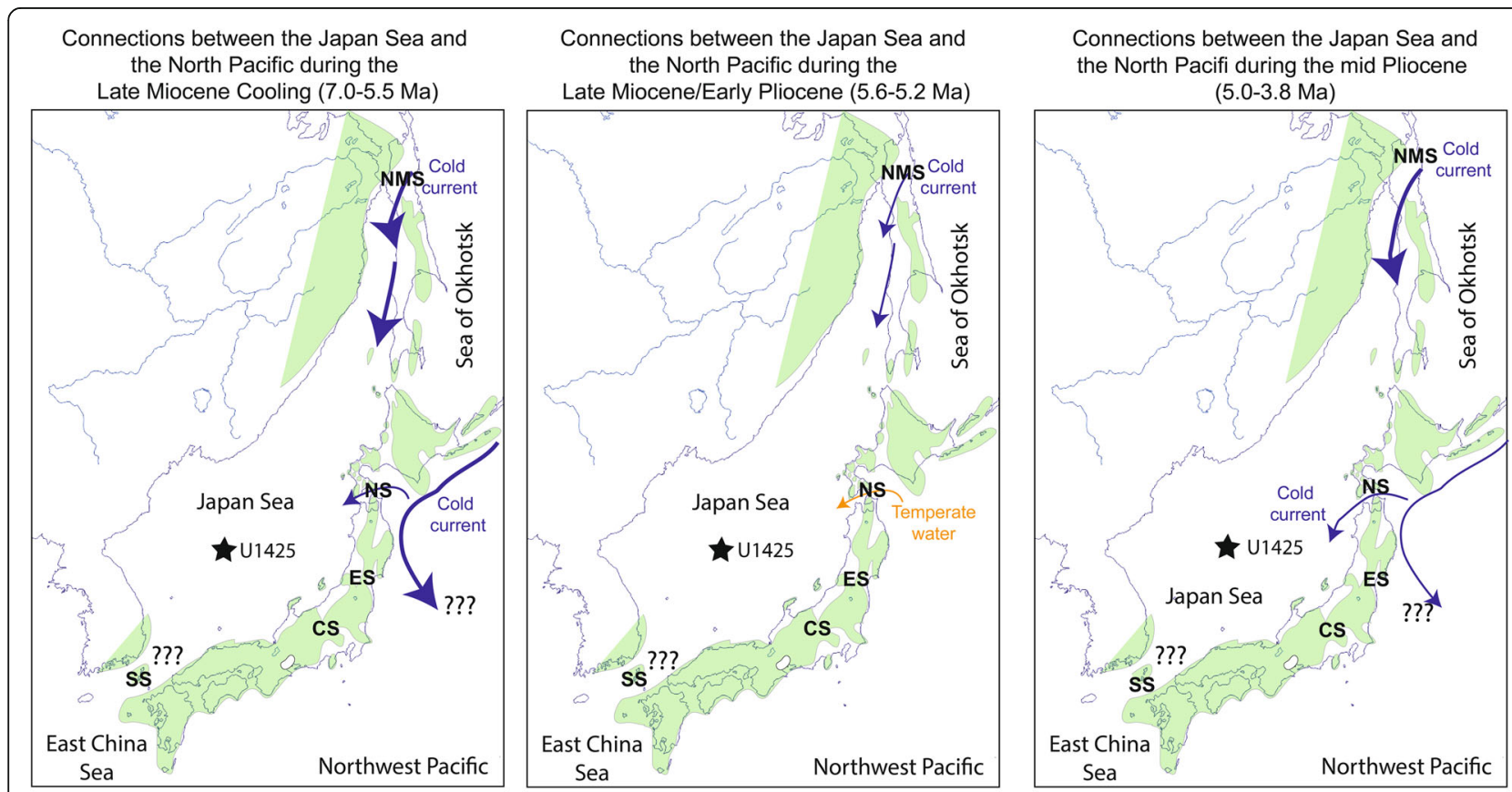

SS: Southern Seaway; CS: Central Seaway; ES: Eastern Seaway; NS: Northern Seaway ; NMS: Northernmost Seaway

Fig. 8 Connections between the Japan Sea and the North Pacific during the late Miocene to late Pliocene 


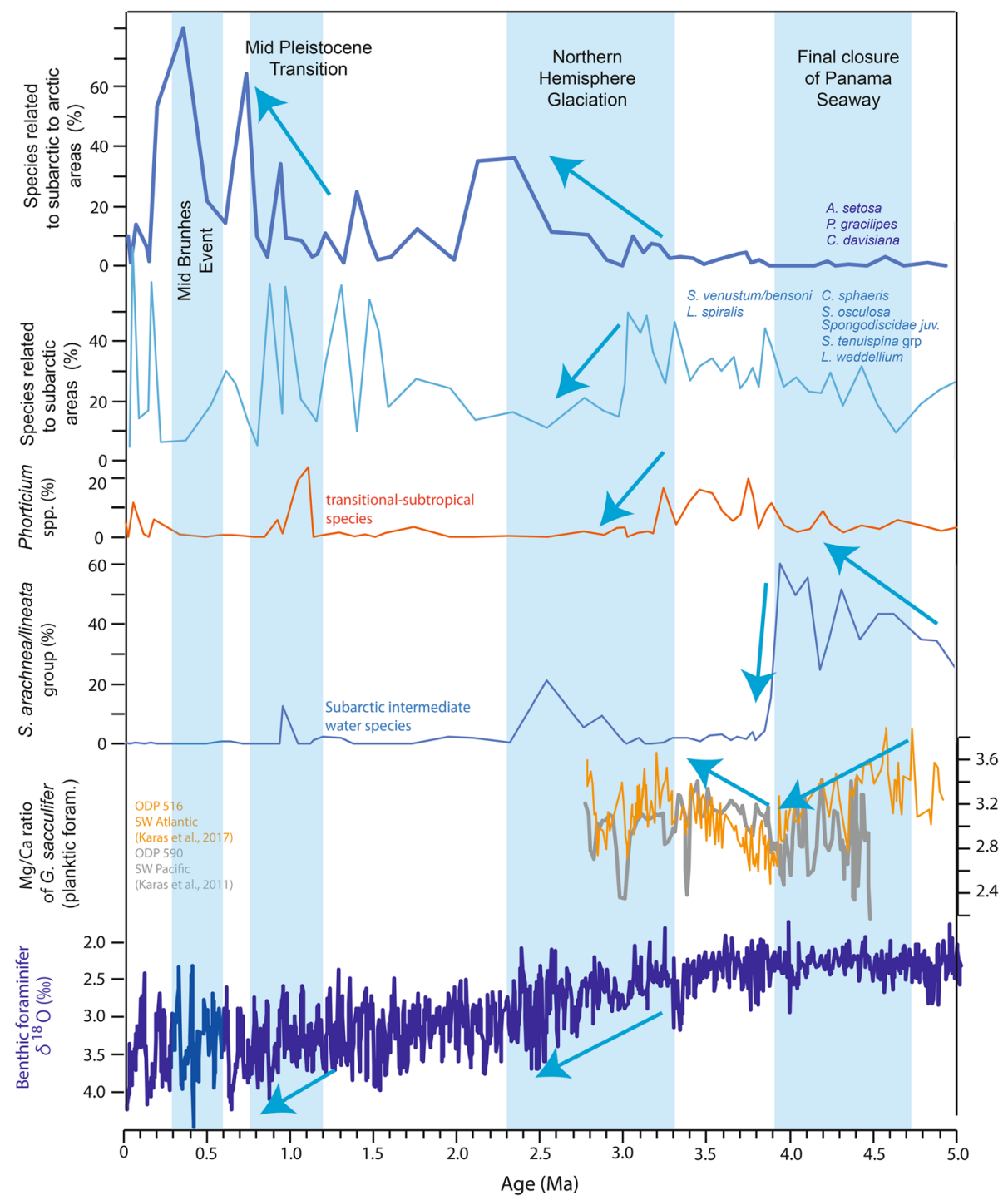

Fig. 9 Details of the paleoceanography of the Japan Sea between 5 and $0 \mathrm{Ma}$. We compare relative abundances of S. arachnea/lineata group, Phorticium spp., and species related to subarctic and arctic areas (the selected species are listed at the upper right in the figure) from Site U1425. These data are compared to Mg/Ca ratio data for plankton of the South Pacific and Atlantic (ODP 516/590, Karas et al. 2011, 2017) and benthic oxygen isotopes (Lisiecki and Raymo 2005)

Pacific. At that time, only the northern seaway, which at a water depth deeper than $300 \mathrm{~m}$ was deep enough to enable inflow of subsurface to intermediate water of the North Pacific, because S. lineata/arachnea inhabits water depths between 300 and $1000 \mathrm{~m}$ in the North Pacific (Kling and Boltovskoy 1995). Therefore, we suggest that such intermediate water influenced the Japan Sea via the northern Strait between 5.0 and 3. 8 Ma (Fig. 8). However, causes and mechanisms are difficult to explain. A re-estimation of $S$. arachnea/lineata group presence using $45-\mu \mathrm{m}$ mesh sieves at other DSDP/ODP sites and comparison with geochemical data would probably provide clarification.
Although it is just speculation, numerous studies have suggested a close link between changes in the North Pacific paleoceanographic conditions and the final shoaling of the Panama Isthmus ca. 5-4 Ma (e.g., Cortese et al. 2004; Haug et al. 2001; Steph et al. 2006; Stroynowski et al. 2015). Steph et al. (2006) showed that the $\delta^{18} \mathrm{O}$ measured in Globigerinoides sacculifer (Brady) (planktic foraminifera) at sites between the Eastern Equatorial Pacific and the Caribbean Sea began to diverge between ca. 4.7 and 4.2 Ma. This was interpreted as a reduced exchange of water masses through the Panama Isthmus, inhibiting exchanges of shallow water between the North Pacific and North Atlantic (Steph et al. 2006). A coupled 
atmosphere-ocean climate model experiment by Motoi et al. (2005) suggested that the closure of the Panama Isthmus not only inhibited the export of warm water from the North Atlantic to the North Pacific but also disturbed the water mass density (salinity) balance of both oceans. In addition, Karas et al. (2017) reconstructed temperatures based on the planktic foraminifera $\mathrm{Mg} / \mathrm{Ca}$ ratio and found a synchronous cooling of the South Atlantic and South Pacific between 5 and 4 Ma. Further, they associated this cooling with an episodic enhanced Atlantic meridional overturning circulation due to water mass density disturbance initiated by the final closure of the Panama Isthmus. In Fig. 9, we plot our radiolarian data against the $\mathrm{Mg} / \mathrm{Ca}$ ratio obtained by Karas et al. (2017) in the South Atlantic and South Pacific. It seems that the interval where the radiolarian assemblage of the Japan Sea is dominated by the $S$. arachnea/lineata group corresponds to the cooling event reported by Karas et al. (2017). Therefore, it is possible that the cooling recorded in the Japan Sea and Northwest Pacific intermediate water is related to the closing of the Panama Isthmus and its related water mass density perturbation, which may have affected the global thermohaline circulation episodically.

\section{Paleoceanography of the Japan Sea during the late Pliocene (ca. 3.8-2.7 Ma)}

The late Pliocene is characterized by a progressive global cooling until the beginning of the NHG (e.g., Haug et al. 1999; Raymo 1994). Abrupt emergence of land is suggested for the Hokkaido and Sakhalin archipelagic water regions based on recent geological studies because of the cooling related to the NHG between 3 and 2 Ma (e.g., Sagayama 2002; Yahata 2002; Kharakhinov 2010). Thus, the exchange of subarctic water via the northernmost seaway would have been affected (Sagayama 2002; Yahata 2002; Kharakhinov 2010). For this interval (3.5$2.7 \mathrm{Ma}$ ), our data show that the transitional-subarctic species and transitional-subtropical species had relative abundances varying between 20 and $40 \%$ and 10 and 20\%, respectively (Fig. 9). Between 3.8 and 2.7 Ma, our data imply that the Japan Sea was probably still influenced by transitional-subarctic and transitional-subtropical water at that time.

Previous studies suggested that the opening of the southern strait at ca. $3 \mathrm{Ma}$ caused only a restricted and localized warming to the southernmost part of the Japan Sea because the channel was probably very narrow and insufficient to supply significant amounts of warm water (e.g., Iijima and Tada 1990; Itaki 2016; Kitamura and Kimoto 2006; Kitamura et al. 2001). Therefore, transitional-subtropical water probably did not flow into the Japan Sea from the southern strait. Koizumi and Yamamoto (2016) showed that at the same latitude, the diatom $T d^{\prime}$-based sea surface temperature (SST) of the Japan Sea (ODP Site 797) was colder than that recorded in the North Pacific (ODP Site 436) at a similar latitude despite the opening of the seaway into the southern Japan Sea at ca. 3.1 Ma (Iijima and Tada 1990; Tada 1994; Ogasawara 1994). To explain this observation, Itaki (2016) suggested the possibility that subarctic water from the Sea of Okhotsk influenced the Japan Sea via a strait west of Hokkaido Island during the Pliocene. Considering the local geological context, we know that until ca. $3 \mathrm{Ma}$ the area around Sakhalin Island was still characterized by shallow neritic facies, implying that cold water of the high latitudes could still influence the Japan Sea via the northernmost seaway of the Japan Sea (Kharakhinov 2010). Therefore, we can infer that transitional-subarctic species probably continued to enter the Japan Sea via a shallow strait between the Sakhalin Islands and the Japan Sea until its closure between 3 and $2 \mathrm{Ma}$ (Kharakhinov 2010), while transitional-subtropical species probably entered through the northern strait, as during the early Pliocene (Figs. 8 and 9).

\section{Paleoceanography of the Japan Sea during the Pleistocene (ca. 2.5-0 Ma)}

At 2.7 Ma, the NHG began, which marked the beginning of the Pleistocene (e.g., Haug et al. 1999; Raymo 1994). At Site U1425, our data show that relative abundances of arctic species increased from $2.7 \mathrm{Ma}$, while transitional-subarctic species and subtropical-transitional species abundances decreased (Fig. 9). In addition, species related to deep water of the North Pacific disappeared (Figs. 3 and 4). This implies a drastic cooling of the Japan Sea due to the NHG, and the absence of Pacific deep-water species suggests that the deep water of the Japan Sea was isolated from the North Pacific because the sill depth of the northern seaway was too shallow. This shoaling is probably due to the sea-level fall caused by the NHG and tectonic uplift of northeastern Japan (e.g., Kamikuri and Motoyama 2007; Itaki 2016).

The Pleistocene is characterized by cyclical glaciation (e.g., Lisiecki and Raymo 2005), and the relationship between the amplitude of glaciations caused by orbital forcing was not linear (e.g., Lisiecki and Raymo 2005; Elderfield et al. 2012). During the early Pleistocene (2.58-1.2 Ma), weak glaciations with a periodicity of $41 \mathrm{ky}$ characterized the glacial-interglacial cycles, while strong glaciations were repeated with a periodicity of nearly $100 \mathrm{ky}$ since the middle Pleistocene $(0.8 \mathrm{Ma})$ (Lisiecki and Raymo 2005; Elderfield et al. 2012). This periodicity shift, called as the mid-Pleistocene transition (MPT) (Berger and Jansen 1994), occurred from 1.2 to 0.8 Ma. During this event, our data show high abundance peaks of species related to arctic shallow water 
between 1.3 and ca. 1.0 and Ma and thus imply that the MPT and its related strong glaciation probably intensified the cooling of the Japan Sea. Additionally, the Mid-Brunhes Event (MBE) occurring between 0.45 and 0.3 Ma (Jansen et al. 1986) corresponds to a period when Earth eccentricity was close to 0 (Berger 1978). This likely caused lower $\mathrm{pCO}_{2}$ and caused a colder spring-summer season, while the autumn/winter season was warmer (Lüthi et al. 2008; Yin and Berger 2010). Our data show high abundance peaks of species related to arctic shallow water between 0.5 and ca. $0.3 \mathrm{Ma}$ (Fig. 9). This suggests intensification of the cooling of the Japan Sea shallow water during these times. Interestingly, this orbital event fit well with our second cooling of the shallow water and thus suggests that the MBE probably intensified the cooling of the Japan Sea.

\section{Conclusions}

Radiolarian assemblages analyzed at Sites U1425 and U1430 enabled us to reconstruct the evolution of the Japan Sea hydrographic system since 9.5 Ma. We can conclude that radiolarians in the Japan Sea were sensitive to environmental changes caused by regional tectonism, as well as global climatic events:

1. During the late Miocene, the sill depth of the northern strait was likely close to $1000 \mathrm{~m}$ until ca. 7.8 Ma and probably enabled inflow of OMZ water from the North Pacific when the sea level was high. Since 7.8 Ma, a progressive shoaling of the northern strait is implied by the radiolarian data, and at about $3 \mathrm{Ma}$, the sill depth of the northern strait was probably $300 \mathrm{~m}$. This shoaling is likely due to enhanced tectonic activity on the Japan islands.

2. The central and eastern seaways were likely the key straits enabling inflow of warm-shallow water of the North Pacific into the Japan Sea between 9.5 and $7 \mathrm{Ma}$, when sea level was high.

3. Our data suggest that C. papillosum is probably related to OMZ water of the North Pacific.

4. Although the habitat zone of $C$. nakasekoi remains difficult to assess, it is possible that $C$. nakasekoi was related to poorly stratified water masses formed when the sea level was low during the late Miocene because the inflow of OMZ and warm water were prevented.

5. The Japan Sea recorded a deep cooling between 7 and $5.5 \mathrm{Ma}$. This cooling is probably related to the late Miocene cooling of Herbert et al. (2016) and enhanced EAWM.

6. A moderate warming occurred between 5.6 and 5.2 Ma in the Japan Sea, and it was probably related to the early Pliocene warming, when an expansion of the tropical warm pool likely occurred

(e.g., Fedorov et al. 2013; Brierley et al. 2009).

7. The Japan Sea subsurface to intermediate water drastically cooled between 5.0 and 4.0 Ma and similar cooling likely occurred in the subarctic area of the Northwest Pacific almost synchronously. The reasons and mechanisms of this cooling remain unknown, but because the timing of this cooling was almost simultaneous with the formation of the Panama Isthmus, they may be related.

8. The NHG and Pleistocene major climatic events, such as the MPT and MBE, intensified the cooling of shallow water in the Japan Sea.

\section{Additional files}

Additional file 1: Raw data (\%) of the species used in this study (U1425). (XLSX $84 \mathrm{~kb}$ )

Additional file 2: Raw data (\%) of the species used in this study (U1430). (XLSX 42 kb)

\section{Abbreviations}

AB: Absolute abundance; CCD: Calcite compensation depth; CCSF: Core composite depth below sea floor; DSDP: Deep-Sea Drilling Program; EAWM: East Asian winter monsoons; F-slide: Faunal slide; IODP: Integrated Ocean Drilling Program; MBE: Mid-Brunhes Event; MIS: Marine isotope stage; MPT: Mid-Pleistocene Transition; NHG: Northern Hemisphere Glaciation;

ODP: Ocean Drilling Program; OMZ: Oxygen minimum zone; Q-slide: Quantitative slide; SD: Standard deviation; SST: Sea surface temperature; TWC: Tsushima Warm Current

\section{Acknowledgements}

We would like to thank the Integrated Ocean Drilling Program (IODP) Expedition 346 for providing us the samples. We also wish to thank the Kochi Core Center (KCC) and its curators for their sampling assistance. We also wish to thank Mr. Shunsuke Kurokawa for discussions about the manuscript and Mr. Yasuaki Chiba for his help in analyzing samples from Site U1430.

\section{Funding}

This work was supported by the Japan Society for the Promotion of Science (JSPS) Research Fellowship for Young Scientists, grant 16J02142 (MKM) and partly founded by the JSPS grant $18 \mathrm{H} 01279$ attributed to Takuya Sagawa (Kanazawa University). This work was also partially financed by the IODP Expedition 346 After Cruise Research Program, JAMSTEC.

Availability of data and materials

The radiolarian data used in this study are available in Additional files 1 and 2, which are attached to this study as supplementary materials.

\section{Authors' contributions}

MKM conducted the treatment and analysis on 154 samples from Site U1425, drew figures and tables, and wrote the manuscript. IT and TR proposed the topic and co-designed the study. KSI conducted analysis on samples from Site U1430. All authors read and approved the final manuscript.

\section{Authors' information}

MKM is a JSPS Research Fellow at the University of Tokyo and now assistant professor at Atmosphere and Ocean Research Institute of the university of Tokyo; IT is a senior researcher at the Geological Survey of Japan, AIST; TR is a professor of the University of Tokyo, the host researcher of MKM, and the co-chief of IODP Expedition 346; KSI is an associate professor at Ibaraki University. 


\section{Competing interests}

The authors declare that they have no competing interests.

\section{Publisher's Note}

Springer Nature remains neutral with regard to jurisdictional claims in published maps and institutional affiliations.

\section{Author details}

'Department of Earth and Planetary Science, Graduate School of Science, The University of Tokyo, 7-3-1 Hongo, Bunkyo-ku, Tokyo 113-0033, Japan. ${ }^{2}$ Present address: Atmosphere and Ocean Research Institute, The University of Tokyo, 5-1-5 Kashiwanoha, Kashiwa, Chiba 277-8564, Japan. ${ }^{3}$ Geological Survey of Japan, AIST, Marine Geology Research Group/Institute of Geology and Geoinformation, Tsukuba Central 7, 1-1-1 Higashi, Tsukuba, Ibaraki 305-8567, Japan. ${ }^{4}$ Faculty of Education, Ibaraki University, 2-1-1 Bunkyo, Ibaraki 310-8512, Japan.

Received: 15 February 2018 Accepted: 14 August 2018 Published online: 18 September 2018

\section{References}

Alexandrovich JM (1992) Radiolarians from Sites 794, 795, and 797 (Japan Sea). In: Tamaki K, Ingle JCJ et al (eds) Proc ODP Sci Res 127/128(1):291-307. Ocean Drilling Program, College Station

Berger A (1978) Long-term variations of daily insolation and quaternary climatic changes. J Atmos Sci 35(12):2362-2367 https://doi.org/10.1175/15200469(1978)035\%3C2362:LTVODI\%3E2.0.CO;2

Berger WH, Jansen E (1994) Mid-Pleistocene climate shift: the Nansen connection. In: Johannessen OM, Muench RD, Overland JE (eds) The polar oceans and their role in shaping the global environment, 295-311. American Geophysical Union, Washington. https://doi.org/10.1029/ GM085p0295

Betzler C, Eberli GP, Lüdmann T, Reolid J, Kroon D, Reijmer JJG, Swart PK, Wright J, Young JR, Alvarez-Zarikian C, Alonso-García M, Bialik OM, Blättler CL, Guo JA, Haffen S, Horozal S, Inoue M, Jovane L, Lanci L, Laya JC, Hui Mee AL, Nakakuni M, Nath BN, Niino K, Petruny LM, Pratiwi SD, Slagle AL, Sloss CR, Su X, Yao Z (2017) Refinement of Miocene Sea level and monsoon events from the sedimentary archive of the Maldives (Indian Ocean). Prog Earth Planet Sci 5:5 https://doi.org/10.1186/s40645-018-0165-x

Biard T, Bigeard E, Audic S, Poulain J, Gutierrez-Rodriguez A, Pesant S, Stemmann L, Not F (2017) Biogeography and diversity of Collodaria (Radiolaria) in the global ocean. ISME J 11(6):1331-1344 https://dx.doi.org/10.1038\%2Fismej. 2017.12

Biard T, Stemmann L, Picheral M, Mayot N, Vandromme P, Hauss H, Gorsky G, Guidi L, Kiko R, Not F (2016) In situ imaging reveals the biomass of giant protists in the global ocean. Nat 532(7600):504-507. https://doi.org/10.1038/ nature 17652

Bjørklund KR, Hatakeda K, Kruglikova SB, Matul AG (2015) Amphimelissa setosa (Cleve) (Polycystina, Nassellaria)-a stratigraphic and paleoecological marker of migrating polar environments in the northern hemisphere during the quaternary. Stratigr 12(1):23-37

Boltovskoy D, Correa N (2016) Biogeography of Radiolaria Polycystina (Protista) in the World Ocean. Prog Oceanogr 149:82-105. https://doi.org/10.1016/j. pocean.2016.09.006

Brierley CM, Fedorov AV, Liu Z, Herbert TD, Lawrence KT, LaRiviere JP (2009) Greatly expanded tropical warm pool and weakened Hadley circulation in the early Pliocene. Sci 323:1714-1718 https://doi.org/10.1126/science. 1167625

Butzin M, Lohmann G, Bickert T (2011) Miocene Ocean circulation inferred from marine carbon cycle modeling combined with benthic isotope records. Paleoceanogr Paleoclimatol 26(1) https://doi.org/10.1029/2009PA001901

Casey R (1982) Lamprocyrtis and Stichocorys lineages: biogeographical and ecological perspectives relating to the tempo and mode of polycystine radiolarian evolution. In: Mamet B, Copeland MJ (eds) third American paleontological convention, proceedings vol I. 77:82

Casey R, McMillen K, Reynolds R, Spaw J, Schwarzer R, Gevirtz H, Bauer M (1979) Relict and expatriated radiolarian fauna in the Gulf of Mexico and its implications. Trans Gulf Coast Assoc Geol Soc 29:224-227

Cerling TE, Harris JM, MacFadden BJ, Leakey MG, Quade J, Eisenmann V, Ehleringer JR (1997) Global vegetation change through the Miocene/ Pliocene boundary. Nature 389(6647):153
Chinzei K (1978) Neogene molluscan faunas in the Japanese Islands: an ecologic and zoogeographic synthesis. Veliger 21(2):155-170

Clift PD (2017) Cenozoic sedimentary records of climate-tectonic coupling in the western Himalaya. Prog Earth Planet Sci 4:39 https://doi.org/10.1186/s40645017-0151-8

Cortese G, Gersonde R, Hillenbrand CD, Kuhn G (2004) Opal sedimentation shifts in the World Ocean over the last 15 Myr. Earth Planet Sci Lett 224(3-4):509-527. https://doi.org/10.1016/j.epsl.2004.05.035

De Wever P, Dumitrica P, Caulet JP, Nigrini C, Caridroit M (2001) Radiolarians in the sedimentary record. Gordon and Breach Science Publishers, Amsterdam

Elderfield H, Ferretti P, Greaves M, Crowhurst S, McCave IN, Hodell DA, Piotrowski AM (2012) Evolution of ocean temperature and ice volume through the midPleistocene climate transition. Sci 337(6095):704-709 https://doi.org/10.1126/ science.1221294

Fedorov AV, Brierley CM, Lawrence KT, Liu Z, Dekens PS, Ravelo AC (2013) Patterns and mechanisms of early Pliocene warmth. Nat 496:43-49 https:// doi.org/10.1038/nature12003

Fujii S, Kaseno Y, Nakagawa T (1992) Neogene paleogeography in the Hokuriku region, Central Japan, based on revised stratigraphic correlation. Mem Geol Soc Jpn 37:85-95

Fukusawa H (1988) Sedimentary mechanism of Neogene bedded siliceous rocks on Late Miocene Wakkanai formation of northern Hokkaido. J Geol Soc Jpn 94:669-688

Gamo T, Nakayama N, Takahata N, Sano Y, Zhang J, Yamasaki E, Taniyasu S, Yamashita N (2014) The sea of Japan and its unique chemistry revealed by time-series observations over the last 30 years. Monogr Environ Earth Planets 2(1):1-22 https://doi.org/10.5047/meep.2014.00201.0001

Hanagata S (2003) Miocene-Pliocene foraminifera from the Niigata oil-fields region, northeastern Japan. Micropaleontol 49(4):293-340 https://doi.org/10. 1661/0026-2803(2003)049[0293:MFFTNO]2.0.CO;2

Hanagata S (2006) Foraminiferal proxies of dissolved oxygen and their changes across the Miocene/Pliocene boundary in the Japan Sea. Stratigr 3:285-306

Hase H, Yoon JH, Koterayama W (1999) The current structure of the Tsushima Warm Current along the Japanese coast. J Oceanogr 55(2):217-235

Haug GH, Sigman DM, Tiedemann R, Pedersen TF, Sarnthein M (1999) Onset of the permanent stratification in the subarctic Pacific Ocean. Nat 401(6755): 779-782. https://doi.org/10.1038/44550

Haug GH, Tiedemann R, Zahn R, Ravelo AC (2001) Role of the Panama uplift on oceanic freshwater balance. Geol 29(3):207-210 https://doi.org/10.1130/00917613(2001)029\%3C0207:ropuoo\%3E2.0.co;2

Herbert TD, Lawrence KT, Tzanova A, Peterson LC, Caballero-Gill R, Kelly CS (2016) Late Miocene global cooling and the rise of modern ecosystems. Nat Geosci 9(11):843-847 https://doi.org/10.1038/ngeo2813

Holbourn A, Kuhnt W, Clemens S, Prell W, Andersen N (2013) Middle to late Miocene stepwise climate cooling: evidence from a high-resolution deep water isotope curve spanning 8 million years. Paleoceanogr Paleoclimatol 28(4):688-699. https://doi.org/10.1002/2013PA002538

Holbourn A, Kuhnt W, Clemens SC, Kochhann KG, Jöhnck J, Lübbers J, Andersen N (2018) Late Miocene climate cooling and intensification of southeast Asian winter monsoon. Nat Commun 9(1):1584. https://doi.org/10.1038/s41467-01803950-1

Holbourn A, Kuhnt W, Lyle M, Schneider L, Romero O, Andersen N (2014) Middle Miocene climate cooling linked to intensification of eastern equatorial Pacific upwelling. Geol 42(1):19-22. https://doi.org/10.1130/G34890.1

Huang Y, Clemens SC, Liu W, Wang Y, Prell WL (2007) Large-scale hydrological change drove the late Miocene C4 plant expansion in the Himalayan foreland and Arabian Peninsula. Geology 35:531-534

lijima A, Tada R (1990) Evolution of tertiary sedimentary basins of Japan in reference to opening of the Japan Sea. J Fac Sci, U Tokyo Section II 22(2): $121-171$

Ikenoue T, Okazaki Y, Takahashi K, Sakamoto T (2016) Bering Sea radiolarian biostratigraphy and paleoceanography at IODP Site U1341 during the last four million years. Deep-Sea Res II Top Stud Oceanogr 125:38-55. https://doi. org/10.1016/j.dsr2.2015.03.004

Irino T, Tada R, Ikehara K, Sagawa T, Karasuda A, Kurokawa S, Seki A, Lu S (2018) Construction of perfectly continuous records of physical properties for darklight sediment sequences collected during IODP Expedition 346. Prog Earth Planet Sci 5:23 https://doi.org/10.1186/s40645-018-0176-7

Itaki T (2003) Elutriation technique for the extraction of radiolarian skeletons from sandy sediments. Fossils 73:38-41 (in Japanese) 
Itaki T (2007) Historical changes of deep-sea radiolarians in the Japan Sea during the last 640 kyrs. Fossils 82:43-51 (in Japanese)

Itaki T (2009) Last glacial to Holocene polycystine radiolarians from the Japan Sea. News Osaka Micropaleontol (NOM) 14:43-89

Itaki T (2016) Transitional changes in microfossil assemblages in the Japan Sea from the late Pliocene to Early Pleistocene related to global climatic and local tectonic events. Prog Earth Planet Sci 3(1):11 https://doi.org/10.1186/ s40645-016-0087-4

Itaki T, Ikehara K, Motoyama I, Hasegawa S (2004) Abrupt ventilation changes in the Japan Sea over the last 30 kyr: evidence from deep-dwelling radiolarians. Palaeogeogr Palaeoclimatol Palaeoecol 208(3-4):263-278 https://doi.org/10. 1016/j.palaeo.2004.03.010

Itaki T, Kimoto K, Hasegawa S (2010) Polycystine radiolarians in the Tsushima Strait in autumn of 2006. Paleontol Res 14(1):19-32 https://doi.org/10.2517/ 1342-8144-14.1.019

Itaki T, Komatsu N, Motoyama I (2007) Orbital- and millennial-scale changes of radiolarian assemblages during the last 220 kyrs in the Japan Sea. Palaeogeogr Palaeoclimatol Palaeoecol 247(1-2):115-130 https://doi.org/10. 1016/j.palaeo.2006.11.025

Jansen JHF, Kuijpers A, Troelstra SR (1986) A mid-Brunhes climatic event: longterm changes in global atmosphere and ocean circulation. Sci 232:619-622 https://doi.org/10.1126/science.232.4750.619

Jolivet L, Tamaki K, Fournier M (1994) Japan Sea, opening history and mechanism: a synthesis. J Geophys Res 99(B11):22237-22259 https://doi.org/10.1029/93JB03463

Kamikuri S (2010) New late Neogene radiolarian species from the middle to high latitudes of the North Pacific. Rev Micropaleontol 53(2):85-106 https://doi. org/10.1016/j.revmic.2008.06.005

Kamikuri S (2012) Evolutionary changes in the biometry of the fossil radiolarian Stichocorys peregrina lineage in the eastern equatorial and eastern North Pacific. Mar Micropaleontol 90-91:13-28 https://doi.org/10.1016/.jmarmicro.2012.04.003

Kamikuri S (2017) Late Neogene radiolarian biostratigraphy of the eastern North Pacific ODP Sites 1020/1021. Paleontol Res 21(3):230-254 https://doi.org/10. 2517/2016PR027

Kamikuri S, Itaki T, Motoyama I, Matsuzaki KM (2017) Radiolarian biostratigraphy during the middle Miocene to quaternary, IODP Sites U1422-U1427 and U1430 in the Japan Sea. Paleontol Res 21(4):397-421 https://doi.org/10.2517/2017PR001

Kamikuri S, Motoyama I (2007) Radiolarian assemblage and environmental changes in the Japan Sea since the Late Miocene. Fossils 82:35-42 (in Japanese)

Kamikuri S, Motoyama I, Nishi H, Iwai M (2009) Neogene radiolarian biostratigraphy and faunal evolution rates in the eastern equatorial Pacific ODP Sites 845 and 1241. Acta Paleontol Pol 54(4):713-742. https://doi.org/10. 4202/ app.2008.0076

Kamikuri S, Motoyama I, Nishimura A (2008) Radiolarian assemblages in surface sediments along longitude $175^{\circ} \mathrm{E}$ in the Pacific Ocean. Mar Micropaleontol 69(2):151-172. https://doi.org/10.1016/j.marmicro.2008.07.005

Kamikuri S, Nishi H, Motoyama I (2007) Effects of late Neogene climatic cooling on North Pacific radiolarian assemblages and oceanographic conditions. Palaeogeogr Palaeoclimatol Palaeoecol 249(3-4):370-392 https://doi.org/10. 1016/j.palaeo.2007.02.008

Kamikuri S, Nishi H, Motoyama I, Saito S (2004) Middle Miocene to Pleistocene radiolarian biostratigraphy in the Northwest Pacific, ocean drilling program leg 186. Island Arc 13(1):191-226. https://doi.org/10.1111/j.1440-1738.2003. 00421.x

Kano K, Kato H, Yanagisawa Y, Yoshida F (1991) Stratigraphy and geologic history of the Cenozoic of Japan. Report no. 274. Geological survey of Japan, Tokyo

Karas C, Nürnberg D, Bahr A, Groeneveld J, Herrle JO, Tiedemann R (2017) Pliocene oceanic seaways and global climate. Sci Rep 7:39842 https://doi. org/10.1038/srep39842

Karas C, Nürnberg D, Tiedemann R, Garbe-Schönberg D (2011) Pliocene Indonesian Throughflow and Leeuwin current dynamics: implications for Indian Ocean polar heat flux. Paleoceanogr Paleoclimatol 26(2) https://doi. org/10.1029/2010PA001949

Kennett JP, Keller G, Srinivasan MS (1985) Miocene planktonic foraminiferal biogeography and paleoceanographic development of the indo-Pacific region. Geol Soc Am Mem 163:197-236

Kharakhinov W (2010) Oil-and-gas geology of the Sakhalin region. Scientific World, Moscow Available at http://www.geokniga.org/bookfiles/geokniganeftegazovaya-geologiya-sahalinskogo-regiona.pdf (in Russian)

Kido Y, Minami I, Tada R, Fujine K, Irino T, Ikehara K, Chun H (2007) Orbital-scale stratigraphy and high-resolution analysis of biogenic components and deepwater oxygenation conditions in the Japan Sea during the last $640 \mathrm{kyr}$.
Palaeogeogr Palaeoclimatol Palaeoecol 247(1):32-49 https://doi.org/10.1016/j. palaeo.2006.11.020

Kitamura A, Kimoto K (2006) History of the inflow of the warm Tsushima current into the sea of Japan between 3.5 and $0.8 \mathrm{Ma}$. Palaeogeogr Palaeoclimatol Palaeoecol 236(3):355-366 https://doi.org/10.1016/j.palaeo.2005.11.015

Kitamura A, Takano O, Takata H, Omote H (2001) Late Pliocene-early Pleistocene paleoceanographic evolution of the sea of Japan. Palaeogeogr Palaeoclimatol Palaeoecol 172(1):81-98 https://doi.org/10.1016/S0031-0182(01)00272-3

Kling SA, Boltovskoy D (1995) Radiolarian vertical distribution patterns across the southern California current. Deep-Sea Res I Oceanogr Res Pap 42(2):191-231 https://doi.org/10.1016/0967-0637(94)00038-T|

Kobayashi I, Tateishi M (1992) Neogene stratigraphy and paleogeography in the Niigata region, Central Japan. Mem Geol Soc Jpn 37:53-70

Koizumi I (1992a) Biostratigraphy and paleoceanography of the Japan Sea based on diatoms: ODP leg 127. In: Tsuchi R, Ingle JC Jr (eds) Pacific Neogene: environment, evolution and events. Univ. Tokyo Press, pp 15-24

Koizumi I (1992b) Diatom biostratigraphy of the Japan Sea: leg 127. In: Pisciotto KA, Ingle JC Jr, von Breymann MT, Barron J et al (eds) Proc ODP Sci Res 127-128(1): 249-289. https://doi.org/10.2973/odp.proc.sr.127128-1.127.1992

Koizumi I, Yamamoto H (2016) Diatom records in the quaternary marine sequences around the Japanese Islands. Quat Int 397:436-447 https://doi. org/10.1016/j.quaint.2015.03.043

Kosaka T, Midori T, Hoyanagi K, Kubota M, Miyahigashi Y (1992) Late Cenozoic stratigraphy and paleoceanographic changes in the northern Fossa magna. Mem Geol Soc Jpn 37:71-83

Lazarus D, Faust K, Popova-Goll I (2005) New species of prunoid radiolarians from the Antarctic Neogene. J Micropalaeontol 24(2):97-121 https://doi.org/10. $1144 / j m .24 .2 .97$

Lee GH, Park SC, Kim DC (2000) Fluctuations of the calcite compensation depth (CCD) in the East Sea (Japan Sea). Geo-Mar Lett 20(1):20-26. https://doi.org/ $10.1007 /$ s003670000029

Ling HY (1992) Radiolarians from the sea of Japan: leg 128. In: Piscotto KA, Ingle JC, von Breymann MT et al (eds) Proc. ODP Sci res 127/128(1):225-236. Ocean drilling program, College Station. https://doi.org/10.2973/odp.proc.sr.127128-1.125.1992

Lisiecki LE, Raymo ME (2005) A Pliocene-Pleistocene stack of 57 globally distributed benthic $\delta^{18} \mathrm{O}$ records. Paleoceanogr Paleoclimatol 20(1):PA1003 https://doi.org/10.1029/2004PA001071

Lombari G, Boden G (1985) Modern radiolarian global distributions. Special publication 16A. Cushman Foundation for Foraminiferal Research, Lawrence, pp 68-69

Lüthi D, Le Floch M, Bereiter B, Blunier T, Barnola J, Siegenthaler U, Raynaud D, Jouzel J, Fischer H, Kawamura K, Stocker T (2008) High-resolution carbon dioxide concentration record 650,000-800,000 years before present. Nat 453(7193):379-382. https://doi.org/10.1038/nature06949

Matsuzaki KM, Itaki T (2016) The effect of boron (B) on radiolarian skeletons during extraction from mudstone and soft sediment. News Osaka Micropaleontol (NOM) 16:75-78

Matsuzaki KM, Itaki T (2017) New Northwest Pacific radiolarian data as a tool to estimate past sea surface and intermediate water temperatures. Paleoceanogr Paleoclimatol 32(3):218-245 https://doi.org/10.1002/2017PA003087

Matsuzaki KM, Itaki T, Kimoto K (2016) Vertical distribution of polycystine radiolarians in the northern East China Sea. Mar Micropaleontol 125:66-84 https://doi.org/10.1016/j.marmicro.2016.03.004

Matsuzaki KM, Nishi H, Suzuki N, Cortese G, Eynaud F, Takashima R, Kawate Y, Sakai T (2014b) Paleoceanographic history of the Northwest Pacific Ocean over the past $740 \mathrm{kyr}$, discerned from radiolarian fauna. Palaeogeogr Palaeoclimatol Palaeoecol 396:26-40 https://doi.org/10.1016/j.palaeo.2013.12.036

Matsuzaki KM, Nishi H, Suzuki N, Kawate Y, Takashima R, Sakai T (2014a) Cycladophora davisiana abundances as a paleoceanographic and stratigraphic tool in high latitude siliciceous sediments. Mar Micropaleontol 106:1-9 https://doi.org/10.1016/j.marmicro.2013.11.002

Matsuzaki KM, Suzuki N (2018) Quaternary radiolarian biostratigraphy in the subarctic northeastern Pacific (IODP Expedition 341 Site U1417) and synchroneity of bioevents across the North Pacific. J Micropalaeontol 37:1-10 https://doi.org/10.5194/jm-37-1-2018

Matsuzaki KM, Suzuki N, Nishi H (2015a) Middle to Upper Pleistocene polycystine radiolarians from hole 902-C9001C, Northwestern Pacific. Paleontol Res 19(s1):1-77 https://doi.org/10.2517/2015PR003

Matsuzaki KM, Suzuki N, Nishi H, Hayashi H, Gyawali BR, Takashima R (2015b) Early to middle Pleistocene paleoceanographic history of southern Japan based on radiolarian data from IODP Exp. 314/315 Sites C0001 and C0002. Mar Micropaleontol 118:17-33 https://doi.org/10.1016/j.marmicro.2015.05.001 
Matul A, Abelmann A (2005) Pleistocene and Holocene distribution of the radiolarian Amphimelissa setosa Cleve in the North Pacific and North Atlantic: evidence for water mass movement. Deep-Sea Res II Top Stud Oceanogr 52(16):2351-2364 https://doi.org/10.1016/j.dsr2.2005.07.008

Matul A, Abelmann A, Tiedemann R, Kaiser A, Nürnberg D (2002) Late Quaternary polycystine radiolarian datum events in the Sea of Okhotsk. Geo-Mar Lett 22(1):25-32 https://doi.org/10.1007/s00367-002-0093-y

McClymont EL, Rosell-Melé A, Haug GH, Lloyd JM (2008) Expansion of subarctic water masses in the North Atlantic and Pacific oceans and implications for midPleistocene ice sheet growth. Paleoceanography and Paleoclimatology 23(4)

Miller KG, Kominz MA, Browning JV, Wright JD, Mountain GS, Katz ME, Sugarman PJ, Cramer BS, Christie-Blick N, Pekar SF (2005) The Phanerozoic record of global sea-level change. Sci 310(5752):1293-1298. https://doi.org/10.1126/ science.1116412

Motoi T, Chan WL, Minobe S, Sumata H (2005) North Pacific halocline and cold climate induced by Panamanian gateway closure in a coupled oceanatmosphere GCM. Geophys Res Lett 32(10):L10618 https://doi.org/10.1029/ 2005GL022844

Motoyama I (1992) Neogene radiolarian stratigraphy in the Tsugaru peninsula Aomori prefecture, northern Japan. News Osaka Micropaleontol 8:89-100

Motoyama I (1993) Late Miocene and Pliocene radiolarian datum levels from DSDP Sites 192 and 302, and hole 438A of mid-to high-latitude NW Pacific. News Osaka Micropaleontol 9:337-347

Motoyama I (1996) Late Neogene radiolarian biostratigraphy in the subarctic Northwest Pacific. Micropaleontol 42(3):221-262. https://doi.org/10.2307/ 1485874

Motoyama I, Maruyama T, Nishimura T, Sakurai K (2016b) Geological age of the whale fossil-bearing calcareous float concretion from the Haw River, Hokkaido, Japan, based on radiolarian and diatom analyses. Bull Hobetsu Mus 31:1-6

Motoyama I, Nakamura S (2002) Radiolarian biostratigraphy of the Miocene Masuporo and Wakkanai formations of the Uruyagawa section, Wakkanai, Hokkaido, Japan, with reference to unconformity. J Geol Soc Jpn 108(4):219-231

Motoyama I, Nishimura A (2005) Distribution of radiolarians in the North Pacific surface sediments along the $175^{\circ}$ E meridian. Paleontol Res 9(2):95-117. https://doi.org/10.2517/prpsj.9.95

Motoyama I, Yamada Y, Hoshiba M, Itaki T (2016a) Radiolarian assemblages in surface sediments of the Japan Sea. Paleontol Res 20(3):176-206 https://doi. org/10.2517/2015PR030

Nigrini C, Lombari G (1984) A guide to Miocene radiolaria. Special publication 22. Cushman Foundation for Foraminiferal Research, Lawrence

Oba T, Kato M, Kitazato H, Koizumi I, Omura A, Sakai T, Takayama T (1991) Paleoenvironmental changes in the Japan Sea during the last 85,000 years. Paleoceanogr 6(4):499-518 https://doi.org/10.1029/91PA00560

Ogasawara K (1994) Neogene paleogeography and marine climate of the Japanese Islands based on shallow-marine molluscs. Palaeogeogr Palaeoclimatol Palaeoecol 108:335-351 https://doi.org/10.1016/00310182(94)90241-0

Ogasawara K, Takano M, Nagato H, Nakano T (2008) Cenozoic molluscan faunas and climatic changes in the Northern Pacific related to Pacific gateways: review and perspective. Bull Geol Surv Jpn 59(7/8):355-364

Okazaki Y, Takahashi K, Itaki T, Kawasaki Y (2004) Comparison of radiolarian vertical distributions in the Okhotsk Sea near the Kuril Island and in the northwestern North Pacific off Hokkaido Island. Mar Micropaleontol 51:57-284. https://doi.org/ 10.1016/j.marmicro.2004.01.003

Oseki S, Suzuki N (2009) Miocene to Pleistocene paleographic distribution of polycystine radiolarians in the North Pacific. News Osaka Micropaleontol (NOM) 14:183-238

Raymo ME (1994) The initiation of the northern hemisphere glaciation. Annu Rev Earth Planet Sci 22:353-383 https://doi.org/10.1146/annurev.ea.22.050194.002033

Raymo ME, Ruddiman WF (1992) Tectonic forcing of late Cenozoic climate. Nat 359(6391):117-122 https://doi.org/10.1038/359117a0

Renz G (1976) The distribution and ecology of Radiolaria in the Central Pacific: plankton and surface sediments. Bull Scripps Inst Oceanogr U Calif 22:1-267

Romine K (1985) Radiolarian biogeography and paleoceanography of the North Pacific at 8 ma. In: Kennett JP (ed) the Miocene Ocean: paleoceanography and biogeography. Geol Soc Am Mem 163:237-273

Ruddiman W (2001) Earth's climate: past and future, 1st edn. Macmillan, New York

Sagayama T (2002) Middle Miocene to Pliocene sedimentary basin analysis of vertical movements in Hokkaido, Japan. Revista Mexicana de Ciencias Geologicas 19(3):215-225
Sandoval MI, Boltovskoy D, Baxter AT, Baumgartner PO (2017) Neogene paleoceanography of the eastern equatorial Pacific based on the radiolarian record of IODP drill sites off Costa Rica. Geochem Geophys Geosyst 18(3): 889-906 https://doi.org/10.1002/2016GC006623

Sato H (1994) The relationship between late Cenozoic tectonic events and stress field and basin development in Northeast Japan. J Geophys Res Solid Earth 99(B1 1):22261-22274 https://doi.org/10.1029/94JB00854

Sato H, Iwasaki T, Kawasaki S, Ikeda Y, Matsuta N, Takeda T, Hirata N, Kawanaka T (2004) Formation and shortening deformation of a back-arc rift basin revealed by deep seismic profiling, Central Japan. Tectonophysics 388(1-4): 47-58 https://doi.org/10.1016/j.tecto.2004.07.004

Schlitzer R (2016) Ocean Data View. http://odv.awi.de

Shen X, Wan S, France-Lanord C, Clift PD, Tada R, Révillon S, Shi X, Zhao D, Liu Y, Yin X, Song Z, Li A (2017) History of Asian eolian input to the sea of Japan since 15 ma: links to Tibetan uplift or global cooling? Earth Planet Sci Lett 474:296-308 https://doi.org/10.1016/j.epsl.2017.06.053

Shinzawa M, Kamikuri S, Motoyama I (2009) Radiolarian fossil from the Miocene series in the Hobetsu area, southern Central Hokkaido, Japan. News Osaka Micropaleontol 14:117-137

Siddall M, Rohling EJ, Almogi-Labin A, Hemleben C, Meischner D, Schmelzer I, Smeed DA (2003) Sea-level fluctuations during the last glacial cycle. Nature 423(6942):853

Steph S, Tiedemann R, Prange M, Groeneveld J, Nurnberg D, Reuning L, Schulz M, Haug GH (2006) Changes in Caribbean surface hydrography during the Pliocene shoaling of the central American seaway. Paleoceanography Paleoclimatol 21:PA4221. https://doi.org/10.1029/2004PA001092

Stroynowski Z, Ravelo AC, Andreasen D (2015) A Pliocene to recent history of the Bering Sea at Site U1430A, IODP Expedition 323. Paleoceanogr 30:1641-1656 https://doi.org/10.1002/2015PA002866

Suzuki N, Not F (2015) Biology and ecology of Radiolaria. In: Ohtsuka S, Suzaki T, Horiguchi T, Suzuki N, Not F (eds) Marine protists. Springer, Tokyo, pp 179-222 https://doi.org/10.1007/978-4-431-55130-0_8

Suzuki U (1989) Geology of Neogene basins in the eastern part of the sea of Japan. Mem Geol Soc Jpn 32:143-183 (in Japanese)

Tada R (1994) Paleoceanographic evolution of the Japan Sea. Palaeogeogr Palaeoclimatol Palaeoecol 108(3-4):487-508 https://doi.org/10.1016/00310182(94)90248-8

Tada R, lijima A (1992) Lithostratigraphy and compositional variation of Neogene hemipelagic sediments in the Japan Sea. In: Tamaki K, Suyehiro K, Allan J, McWilliams M et al (eds) Proc ODP Sci Res 127/128(2):1229-1260. https://doi. org/10.2973/odp.proc.sr.127128-2.188.1992

Tada R, Irino T, Ikehara K, Karasuda A, Sugisaki S, Xuan C, Sagawa T, Itaki T, Kubota Y, Lu S, Seki A, Murray RW, Alvarez-Zarikian C et al (2018) High-resolution and high-precision correlation of dark and light layers in the quaternary hemipelagic sediments of the Japan Sea recovered during IODP Expedition 346. Prog Earth Planet Sci 5:19 https://doi.org/10.1186/s40645-018-0167-8

Tada R, Irino T, Koizumi I (1999) Land-ocean linkages over orbital and millennial timescales recorded in Late Quaternary sediments of the Japan Sea. Paleoceanogr 14(2):236-247 https://doi.org/10.1029/1998PA900016

Tada R, Murray RW, Alvarez Zarikian CA, Anderson WT Jr, Bassetti MA, Brace BJ, Clemens SC, da Costa Gurgel MH, Dickens GR, Dunlea AG, Gallagher SJ, Giosan L, Henderson ACG, Holbourn AE, Ikehara K, Irino T, Itaki T, Karasuda A, Kinsley CW, Kubota Y, Lee GS, Lee KE, Lofi J, Lopes C, Peterson LC, SaavedraPellitero M, Sagawa T, Singh RK, Sugisaki S, Toucanne S, Wan S, Xuan C, Zheng H, Ziegler M (2015a) Site U1425. In: Tada R, Murray RW, Alvarez Zarikian CA et al (eds) Proc. IODP vol 346. Ocean drilling program, College Station. https://doi.org/10.2204/iodp.proc.346.106.2015

Tada R, Murray RW, Alvarez Zarikian CA, Anderson WT Jr, Bassetti MA, Brace BJ, Clemens SC, da Costa Gurgel MH, Dickens GR, Dunlea AG, Gallagher SJ, Giosan L, Henderson ACG, Holbourn AE, Ikehara K, Irino T, Itaki T, Karasuda A, Kinsley CW, Kubota Y, Lee GS, Lee KE, Lofi J, Lopes C, Peterson LC, Saavedra-Pellitero M, Sagawa T, Singh RK, Sugisaki S, Toucanne S, Wan S, Xuan C, Zheng H, Ziegler M (2015b) Site U1430. In: Tada R, Murray RW, Alvarez Zarikian CA et al (eds) Proc. IODP vol 346. Integrated Ocean Drilling Program, College Station. https://doi.org/10. 2204/iodp.proc.346.110.2015

Tada R, Zheng H, Clift PD (2016) Evolution and variability of the Asian monsoon and its potential linkage with uplift of the Himalaya and Tibetan plateau. Prog Earth Planet Sci 3:4. https://doi.org/10.1186/s40645-016-0080-y

Taira A (2001) Tectonic evolution of the Japanese island arc system. Annu Rev Earth Planet Sci 29:109-134. https://doi.org/10.1146/annurev.earth.29.1.109 
Takahashi K (1991) Radiolaria: flux, ecology, and taxonomy in the Pacific and Atlantic. Ocean Biocoenosis Ser 3:1-301

Takahashi K, Honjo S (1981) Vertical flux of Radiolaria: a taxon-quantitative sediment trap study from the western tropical Atlantic. Micropaleontol 7 : 140-190. https://doi.org/10.2307/1485284

Takeuchi T, Kodama K, Ozawa T (1999) Paleomagnetic evidence for block rotations in Central Hokkaido-South Sakhalin, Northeast Asia. Earth Planet Sci Lett 169(1):7-21 https://doi.org/10.1016/S0012-821X(99)00081-3

Tamaki T, Suyehiro K, Allan J, Ingle JC, Pisciotto KA (1992) Tectonic synthesis and implication of Japan Sea ODP drilling. In: Tamaki K, Suyehiro K, Allan J, McWilliams M et al (eds) Proc ODP Sci res 127/128(2):1333-1348. Ocean Drilling Program, College Station. https:/doi.org/10.2973/odp.proc.sr.127128-2.240.1992

Tanaka S, Takahashi K (2008) Detailed vertical distribution of radiolarian assemblage (0-3000 m, fifteen layers) in the central subarctic Pacific, June 2006. Mem Fac Kyushu Univ Ser D, Earth \& Planet Sci XXXIII(1):49-72

Ujiie H, Ichikura M (1973) Holocene to uppermost Pleistocene in a piston core from off San'in district, sea of Japan. Trans Proc Soc Jpn 91:137-150 https:// doi.org/10.14825/prpsj1951.1973.91_137

Wang P, Li Q, Li CF (2014) Geology of the China seas (Vol. 6), 1st edn. Elsevier, Amsterdam

Watanabe S, Tada R, Ikehara K, Fujine K, Kido Y (2007) Sediment fabrics, oxygenation history, and circulation modes of Japan Sea during the Late Quaternary. Palaeogeogr Palaeoclimatol Palaeoecol 247:50-67. https://doi. org/10.1016/j.palaeo.2006.11.021

White LD, Alexandrovich JM (1992) Pliocene and Pleistocene abundance and preservation of siliceous microfossil assemblages from Sites 794, 795, and 797: implications for circulation and productivity in the Japan Sea. In: Pisciotto KA, Ingle JC Jr, von Breymann MT, Barron J et al (eds) Proc ODP Sci res 127/128(1):341-357. Ocean drilling program, College Station. https://doi. org/10.2973/odp.proc.sr.127128-1.140.1992

Woodruff F, Savin SM (1989) Miocene Deepwater oceanography. Paleoceanogr 4(1):87-140 https://doi.org/10.1029/PA004i001 p00087

Yahata M (2002) Evolution in space and time of Late Cenozoic hydrothermal activity and ore mineralization in Hokkaido, Japan. Report No. 73, Geological Survey of Hokkaido, pp 151-194

Yamamoto M, Naraoka H, Ishiwatari R, Ogihara S (2005) Carbon isotope signatures of bacterial 28-norhopanoic acids in Miocene-Pliocene diatomaceous and phosphatic sediments. Chem Geol 218(1-2):117-133 https://doi.org/10.1016/j.chemgeo.2005.01.027

Yin Q, Berger A (2010) Insolation and $\mathrm{CO}_{2}$ contribution to the interglacial climate before and after the mid-Brunhes event. Nat Geosci 3(4):243-246 https://doi. org/10.1038/ngeo 771

Yokoyama Y, De Deckker P, Lambeck K, Johnston P, Fifield LK (2001) Sea-level at the Last Glacial Maximum: evidence from northwestern Australia to constrain ice volumes for oxygen isotope stage 2. Palaeogeography, Palaeoclimatology. Palaeoecology 165(3-4):281-297

Yoshida T, Kimura JI, Yamada R, Acocella V, Sato H, Zhao D, Nakajima J, Hasegawa A, Okada T, Honda S, Ishikawa M, Dicky O, Prima A, Kudo T, Shibazaki B, Tanaka A, Ishikawa M (2013) Evolution of late Cenozoic magmatism and the crust-mantle structure in the NE Japan Arc. Geol Soc Lond, Spec Publ 385:SP385-SP315 https://doi.org/10.1144/SP385.15

Zachos J, Dickens GR, Zeebe RE (2008) An early Cenozoic perspective on greenhouse warming and carbon-cycle dynamics. Nat 451(17):279-283 https://doi.org/10.1038/nature06588

Zachos J, Pagani M, Sloan L, Thomas E, Billups K (2001) Trends, rhythms, and aberrations in global climate $65 \mathrm{Ma}$ to present. Sci 292:686-693. https://doi. org/10.1126/science.1059412

Zhang L, Suzuki N (2017) Taxonomy and species diversity of Holocene pylonioid radiolarians from surface sediments of the northeastern Indian Ocean. Palaeontol Electronica 20.3(2017):1-68 https://doi.org/10.26879/718

Zhang L, Suzuki N, Nakamura Y, Tuji A (2018) Modern shallow water radiolarians with photosynthetic microbiota in the western North Pacific. Mar Micropaleontol 139:1-27 https://doi.org/10.1016/..marmicro.2017.10.007

\section{Submit your manuscript to a SpringerOpen ${ }^{\circ}$ journal and benefit from:}

- Convenient online submission

- Rigorous peer review

- Open access: articles freely available online

- High visibility within the field

- Retaining the copyright to your article

Submit your next manuscript at $\boldsymbol{\nabla}$ springeropen.com 